Schmerz

https://doi.org/10.1007/s00482-020-00472-y

(c) Springer Medizin Verlag $\mathrm{GmbH}$, ein Teil von Springer Nature 2020

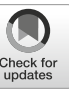

Winfried Häuser, ${ }^{1,2}$ Frietjof Bock $\cdot$ Michael Hüppe ${ }^{4}$ Monika Nothacker ${ }^{5}$.

Heike Norda ${ }^{6} \cdot$ Lukas Radbruch $^{7} \cdot$ Marcus Schiltenwolf $^{8} \cdot$ Matthias Schuler $^{9}$. Thomas Tölle $^{10} \cdot$ Annika Viniol $^{11} \cdot$ Frank Petzke $^{12} \cdot$ Koautoren für die Konsensusgruppe der 2 . Aktualisierung der S3-Leitlinie LONTS

'Medizinisches Versorgungszentrum für Schmerzmedizin und seelische Gesundheit, Saarbrücken St. Johann, Saarbrücken, Deutschland; ${ }^{2}$ Klinik für Psychosomatische Medizin und Psychotherapie, Technische Universität München, München, Deutschland; ${ }^{3}$ Orthopädie am grünen Turm Ravensburg, Ravensburg, Deutschland; ${ }^{4}$ Klinik für Anästhesiologie und Intensivmedizin, Universität zu Lübeck, Lübeck, Deutschland; ${ }^{5}$ AWMF-Institut für Wissensmanagement Berlin, Berlin, Deutschland; ${ }^{6}$ SchmerzLOS, Lübeck, Deutschland; ${ }^{7}$ Klinik für Palliativmedizin, Universitätsklinikum Bonn, Bonn, Deutschland; ${ }^{8}$ Konservative Orthopädie/Schmerztherapie, Ambulanz und Tagesklinik, Universitätsklinikum Heidelberg, Heidelberg, Deutschland; ${ }^{9}$ Klinik für Geriatrie und Intensivmedizin, Diakonissenkrankenhaus Mannheim, Mannheim, Deutschland; ${ }^{10} \mathrm{Klinik}$ für Neurologie, Technische Universität München, München, Deutschland; "Abteilung für Allgemeinmedizin, Präventive und Rehabilitative Medizin, Philipps-Universität Marburg, Marburg, Deutschland; ${ }^{12}$ Schmerzmedizin, Klinik für Anästhesiologie, Universitätsmedizin Göttingen, Göttingen, Deutschland

\title{
Empfehlungen der zweiten Aktualisierung der Leitlinie LONTS
}

\section{Langzeitanwendung von Opioiden bei chronischen nicht-tumorbedingten Schmerzen}

Koautoren für die Konsensusgruppe der 2. Aktualisierung der S3-Leitlinie LONTS

Karl-Jürgen Bär, Christoph Baerwald, Matthias Beintker, Jens Büntzel, Corinna Elling-Audersch, Stephan Freys, Irmela Gnass, Ursula Havemann-Reinecke, Kirstin Hupfer, Ulrich Kellner, Claas Lahmann, Martin Marziniak, Gerhard Müller, Holger Petri, Achim Rody, Michael Schäfer, Dietmar Schöffel, Volker Thieme, Volker Tronnier, Dirk Wolter, Dan Ziegler

\section{Einleitung}

Die Opioidepidemie in Nordamerika (steigende Verordnungen von Opioiden für Menschen, assoziiert mit einem Anstieg der missbräuchlichen/abhängigen Verwendung, notfallmäßigen Krankenhausaufnahmen wegen Überdosierungen und Todesfällen mit verordneten Opioiden) [112] weist auf die Notwendigkeit hin, den Stellenwert von Opioiden in der Therapie von chronischen nichttumorbedingten Schmerzen (CNTS) kritisch zu überprüfen. Die planmäBige Aktualisierung der Leitlinie war aufgrund des Regelwerks der AWMF notwendig. Die erste Aktualisierung der Leitlinie war bis Oktober 2019 gültig [82]. Weiterhin hatten die sporadischen Literatursuchen (der Evidenzgruppe der Leitlinie) neue randomisierte, kontrollierte Studien mit Opioiden bei CNTSSyndromen gefunden, welche nach der ersten Aktualisierung der Leitlinie [82] erschienen waren.

\section{Methoden}

Bezüglich der Methoden der Erstellung der Empfehlungen wird auf den Leitlinienreport in dieser Ausgabe (https:// doi.org/10.1007/s00482-020-00471-z) verwiesen.

\section{Ergebnisse}

Die Kernempfehlungen der Leitlinie sind fett hervorgehoben. Die Stärke des Konsens wird in doppelter Form angegeben: alle Mitglieder der Konsensusgruppe; Konsensusgruppe mit Ausschluss der Mitglieder mit moderaten Interessenkonflikten. Bei Empfehlungen, die aus der 2014-Version der Leitlinie übernommen wurden, wird die Stärke des Konsens nicht in Prozentzahlen angegeben.

\section{Präambel}

\section{Definition von opioidhaltigen Analgetika}

Geprüft 2019. Konsensbasierte Feststellung: Aus Gründen der sprachlichen Einfachheit wird in der Leitlinie der Begriff opioidhaltige Analgetika verwendet. Starker Konsens

Kommentar: Die Leitlinie nimmt zu selektiven Opioidagonisten mit und ohne Kombination mit Substanzen zur 
Reduktion von Obstipation und missbräuchlicher Verwendung sowie zu Substanzen mit gemischten opioidergen und nicht-opioidergen Wirkmechanismen im Vergleich zu Placebo und nichtopioidhaltigen Analgetika Stellung. Bei den Substanzen mit gemischten opioidergen/und nicht-opioidergen Wirkmechanismen werden in der Leitlinie die Substanzen Buprenorphin (ein Opioid mit agonistischer Wirkung am $\mu$-Rezeptor und antagonistischer Wirkung am k-Rezeptor), Tramadol ( $\mu$-OpioidrezeptorAgonist und Wiederaufnahmehemmer von Noradrenalin und Serotonin) und Tapentadol ( $\mu$-Opioidrezeptor-Agonist und Wiederaufnahmehemmer von Noradrenalin) behandelt. Weitere Substanzen mit gemischten opioidergen/und nicht-opioidergen Wirkmechanismen (z.B. Ketamin) werden nicht behandelt, da sie in der Langzeittherapie mit Opioiden in Deutschland nicht eingesetzt werden. Weiterhin nimmt die Leitlinie nicht Stellung zu Methadon und L-Polamidon, da diese Substanzen in Deutschland überwiegend in der Substitutionsbehandlung eingesetzt werden.

Mischpräparate aus Opioiden und Nicht-Opioiden (z. B. Codein + Paracetamol, Tramadol + Paracetamol) werden in den Metaanalysen und Empfehlungen der Leitlinie nicht berücksichtigt, da sich bei Analysen der Wirksamkeit und Sicherheit die Effekte von Opioiden nicht von den Effekten der Kombinationsmedikamente unterscheiden lassen.

\section{Definition Langzeitanwendung}

2.1 Neu 2019. Evidenzbasierte Feststellung: Es gibt keine einheitliche, international anerkannte Definition einer Langzeitanwendung bzw. Langzeittherapie von opioidhaltigen Analgetika. Starker Konsens $(100 \% ; 100 \%)$

2.2 Neu 2019. Konsensbasierte Feststellung: Bei evidenzbasierten Empfehlungen wird in der Leitlinie zwischen folgenden Zeitdauern unterschieden: Kurzfristig: 4-12 Wochen; mittelfristig: 13-26 Wochen; langfristig: $\geq 26$ Wochen. Starker Konsens (100\%; 97\%)

2.3 Geprüft 2019. Konsensbasierte Feststellung: Eine Langzeitanwendung von opioidhaltigen Analgetika wird aus klinischer Sicht bei einer Therapiedauer von $>3$ Monaten angenommen. Starker Konsens

Kommentar: Zur Definition der Langzeitanwendung wurde eine Suche in PubMed mit den Suchbegriffen „Long-term opioid therapy AND definition" (13 Treffer) durchgeführt und die Definitionen einer Langzeitanwendung in den durch die systematische Literatursuche identifizierten Leitlinien überprüft. Es gibt keine international einheitlich verwendete Definition einer Langzeittherapie mit opioidhaltigen Analgetika. Die American Pain Society definierte in ihren „Clinical Guidelines for the Use of Chronic Opioid Therapy in Chronic Noncancer Pain“" eine Langzeitanwendung von Opioiden als „Use of opioids to treat chronic pain on a daily basis, or near daily, for at least 90 days and often indefinitely [149]“.

Die französische Leitlinie zu Opioiden bei CNTS unterscheidet eine Therapiedauer von <3 Monaten, 3-6 Monaten und 6-12 Monaten. Eine „Fortsetzung“ („poursuivre un opioïde") einer Opioidtherapie wird ab einem Zeitraum von drei Monaten angenommen [103]. In den Metaanalysen der kanadischen Leitlinie wird eine chronische Opioidtherapie als Therapie länger als drei Monate definiert [21]. In den Analysen, welche der US-amerikanischen Leitlinie zugrunde gelegt wurden, wurde eine Langzeittherapie ab einer Therapiedauer von $>3 \mathrm{bzw}$. $>12$ Monaten angenommen [32, 59]. In Übereinstimmung mit dem Vorgehen in einigen US-amerikanischen Versorgungsforschungsstudien wurde in zwei deutschen Studien mit Krankenkassendaten eine Langzeitanwendung durch mindestens eine Verschreibung von Opioiden pro Quartal in mindestens drei zusammenhängenden Quartalen während eines Jahres definiert [83, 100]. In Cochrane-Metaanalysen von randomisierten, kontrollierten Studien wurden als Kriterium für eine längerfristige Therapie mit opioidhaltigen Analgetika folgende Zeiträume gewählt: ,shortterm“ (kurzfristig): 4-12 Wochen; ,,intermediate" (mittelfristig): 13-26 Wochen; "long-term“ (langfristig oder Langzeittherapie) $>26$ Wochen [29]. „Very shortterm" ( $<4$ Wochen) [29] Studien wurden für die Metaanalysen nicht berücksichtigt. Eine $<4$-wöchige Therapie ist eher der Akutschmerztherapie zuzuordnen.

Die Therapiedauer wird in den für die Leitlinie erstellten systematischen Übersichtsarbeiten und Metaanalysen durch die Summe der Tage der doppelblinden Titrations- und Erhaltungsphase (im Falle von Parallel- bzw. Cross-over-Design) bzw. Absetzphase („withdrawal“) (im Falle von Studien mit Enriched-enrolment-randomized-withdrawal[EERW]Design) definiert. Auswasch-, unverblindete Start- („run-in“), Ausschleich- („tapering") und Nachbeobachtungsphasen werden nicht in die Therapiedauer eingerechnet.

In der Praxis der Opioidtherapie (siehe Kapitel III) wird bei einer Therapiedauer $\geq 3$ Monaten eine Langzeitanwendung bzw. -therapie angenommen.

\section{Ziel der Aktualisierung der Leitlinie}

Geprüft 2019. Konsensbasierte Feststellung: Ziel der Aktualisierung der Leitlinie ist die Förderung eines verantwortungsvollen Umgangs von Ärzten, anderen an der Behandlung beteiligten Personen des Gesundheitswesens und Patienten mit opioidhaltigen Analgetika in der Langzeitanwendung des chronischen Nichttumorschmerzes durch

- Benennen von möglichen Indikationen und Kontraindikationen einer Therapie mit opioidhaltigen Analgetika, - praxisbezogene Hinweise für die Durchführung und Beendigung einer opioidhaltigen Analgetikatherapie.

\section{Starker Konsens}

\section{Themen der Leitlinie}

Geprüft 2019. Konsensbasierte Feststellung: Die Leitlinie nimmt zu Indikationen und Kontraindikationen sowie zur Durchführung einer $\geq 4$-wöchigen Therapie mit opioidhaltigen Analgetika Stellung. Bezüglich des Stellenwerts von opioidhaltigen Analgetika im Vergleich zu anderen medikamentösen sowie nicht-medikamentösen Therapieoptionen bei chronischen Schmerzsyndromen verweist die Leitlinie auf deutsche S3-Leitlinien zu den jeweiligen Krankheitsbildern und falls solche nicht vorliegen - auf interna- 
Schmerz https://doi.org/10.1007/s00482-020-00472-y

(c) Springer Medizin Verlag GmbH, ein Teil von Springer Nature 2020

\section{W. Häuser $\cdot$ F. Bock $\cdot$ M. Hüppe $\cdot$ M. Nothacker $\cdot$ H. Norda $\cdot$ L. Radbruch $\cdot$ M. Schiltenwolf $\cdot$ M. Schuler $\cdot$ T. Tölle $\cdot$ A. Viniol $\cdot$ F. Petzke $\cdot$ Koautoren für die Konsensusgruppe der 2. Aktualisierung der S3-Leitlinie LONTS \\ Empfehlungen der zweiten Aktualisierung der Leitlinie LONTS. Langzeitanwendung von Opioiden bei chronischen nicht-tumorbedingten Schmerzen}

\section{Zusammenfassung}

Hintergrund. Die planmäßige zweite Aktualisierung der S3-Leitlinie LONTS (,Langzeitanwendung von Opioiden bei chronischen nicht-tumorbedingten Schmerzen" [CNTS]), AWMF(Arbeitsgemeinschaft der Wissenschaftlichen Medizinischen Fachgesellschaften)-Registernummer 145003, wurde ab Dezember 2018 vorgenommen. Methodik. Die Leitlinie wurde unter Koordination der Deutschen Schmerzgesellschaft von 28 wissenschaftlichen Fachgesellschaften und zwei Patientenselbsthilfeorganisationen entwickelt. Die Literaturrecherche erfolgte über die Datenbanken CENTRAL, MEDLINE und Scopus (bis Dezember 2018). Die systematischen Übersichtsarbeiten und Metaanalysen von randomisierten, kontrollierten Studien zur Wirksamkeit und Sicherheit von Opioiden beim CNTS der früheren Versionen der Leitlinie wurden aktualisiert. Die Graduierung der Evidenzstärke erfolgte nach dem Schema des Oxford Centre for Evidence-Based Medicine. Die Formulierung und Graduierung der Empfehlungen erfolgte in einem mehrstufigen, formalisierten Konsensusverfahren nach dem Regelwerk der AWMF. Die Leitlinie wurde von vier externen Schmerzmedizinern begutachtet. Eine öffentliche Kommentierung war über vier Wochen möglich.

Ergebnisse. Opioidhaltige Analgetika sind eine medikamentöse Therapieoption in der kurz- (4-12 Wochen), mittel- (13-25 Wochen) und langfristigen Therapie ( $\geq 26$ Wochen) von chronischen Arthrose- und Rückenschmerzen sowie chronischen Schmerzen bei diabetischer Polyneuropathie und Postzosterneuralgie. Kontraindikationen sind primäre Kopfschmerzen sowie funktionelle und psychische Störungen mit dem (Leit-)Symptom Schmerz. Die Leitlinie nennt weitere Krankheitsbilder, bei denen ein individueller Therapieversuch erwogen werden kann. Eine Langzeittherapie mit Opioiden beim CNTS ist mit relevanten Risiken verbunden.

Schlussfolgerung. Ein verantwortungsvoller Einsatz von Opioiden verlangt die Berücksichtigung möglicher Indikationen und Kontraindikationen sowie eine regelmäßige Erfassung von Wirksamkeit und Nebenwirkungen. Opioide bleiben eine Behandlungsoption für CNTS, wenn nicht-medikamentöse Therapien nicht ausreichend wirksam sind und/oder medikamentöse Alternativen nicht wirksam waren oder nicht vertragen wurden oder kontraindiziert sind.

\section{Schlüsselwörter}

Opioide - Chronischer nicht-tumorbedingter Schmerz · Systematische Übersicht . Metaanalysen - Leitlinie

\section{Recommendations of the second update of the LONTS guidelines. Long-term opioid therapy for chronic noncancer pain}

\section{Abstract}

Background. The second scheduled update of the German S3 guidelines on long-term opioid therapy for chronic noncancer pain (CNCP), the LONTS (AWMF registration number 145/003), was started in December 2018. Methods. The guidelines were developed by 28 scientific societies and 2 patient self-help organizations under the coordination of the German Pain Society. A systematic literature search in the Cochrane Central Register of Controlled Trials (CENTRAL), MEDLINE and Scopus databases (up until December 2018) was performed. The systematic reviews with meta-analyses of randomized controlled trials with opioids for CNCP from the previous versions of the guideline were updated. Levels of evidence were assigned according to the classification system of the Oxford Centre for Evidence-Based Medicine. The strength of the recommendations was established by formal multistep procedures in order to reach a consensus according to the Association of the Medical Scientific Societies in Germany (AWMF) regulations. The guidelines were reviewed by four external pain physicians. Public comments were possible for 4 weeks. Results. Opioid-based analgesics are a drug-based treatment option for shortterm (4-12 weeks), intermediate-term (13-25 weeks) and long-term ( $\geq 26$ weeks) therapy of chronic osteoarthritis, diabetic polyneuropathy, postherpetic neuralgia and low back pain. Contraindications are primary headaches as well as functional somatic syndromes and mental disorders with the (cardinal) symptom pain. Based on a clinical consensus the guidelines list other medica conditions for which a therapy with opioids can be considered on an individual basis Long-term therapy of CNCP with opioids is associated with relevant risks.

Conclusion. A responsible administration of opioids requires consideration of possible indications and contraindications as well as regular assessment of efficacy and adverse effects. Opioids remain a treatment option for CNCP if nonpharmacological therapies are not effective and/or other drugs are not effective, are not tolerated or are contraindicated.

\section{Keywords}

Opioids - Chronic noncancer pain - Systematic review · Meta-analyses · Guidelines tionale Leitlinien, welche dem deutschen S3-Niveau entsprechen. Starker Konsens

\section{Neu 2019}

Entwicklung der Langzeitverordnungen von opioidhaltigen Analgetika für
Patienten mit nicht-tumorbedingten Schmerzen in Deutschland. Evidenzbasierte Feststellungen:

a) Im Zeitraum von 2006 bis 2016 gab es einen Anstieg der Einzelverordnungen von Opioiden von $4,2 \%$ auf $4,9 \%$ in der deutschen Gesamtbevölkerung. Seit 2016 haben die Gesamtverordnungen von opioidhaltigen Analgetika in der deutschen Gesamtbevölkerung nicht zugenommen. EL3b. Starker Konsens (100\%; 100\%) 
b) Die Langzeitverordnungen (mindestens drei zusammenhängende Quartale) von opioidhaltigen Analgetika für Patienten mit nicht-tumorbedingten Schmerzen in Deutschland haben seit 2012 nicht zugenommen. EL2b. (100\%; $100 \%)$

\section{Kommentar:}

a) Nach den Daten des „Norddeutschen Apothekenrechenzentrums“ (Erfassung der Verschreibungsdaten von 11 Mio. Krankenversicherten erfasst über $80 \%$ der Apotheken in Norddeutschland) stieg der Anteil der Langzeitverordnungen von Opioiden von 2006 bis 2009 von 19,2\% auf $21,2 \%$ [22]. Von 2009 bis $2015 \mathrm{kam}$ es zu einem geringen kontinuierlichen Rückgang der verschriebenen Opioidtagesdosen (107 auf 102 DDD) [23]. Laut Arzneiverordnungsreport kam es von 2009 bis 2016 zu einem Anstieg der Gesamtverordnungen von Opioiden von 9\%. Die Gesamtverordnungen haben in den Jahren 2016-2018 nicht mehr zugenommen [15].

Die genannten Studien differenzierten nicht zwischen Verschreibungen für tumor- und nicht-tumorbedingte Schmerzen. Aussagen zur individuellen Dosierung und Länge einer Behandlung können nicht abgeleitet werden.

b) 1,3\% der Versicherten der BEK mit nicht-tumorbedingten Schmerzen erhielten im Jahr 2012 eine Langzeitopioidtherapie („long-term opioid therapy“ [LTOT]) [100]. Eine LTOT wurde durch mindestens eine Verschreibung von Opioiden pro Quartal in mindestens drei aufeinanderfolgenden Quartalen definiert. 0,8\% der Versicherten mit nicht-tumorbedingten Schmerzen von 69 deutschen gesetzlichen Krankenkassen (standardisiert bzgl. Alter und Geschlecht auf die allgemeine deutsche Bevölkerung) erhielten im Jahr 2014 eine LTOT [83].

Die Anzahl und Dauer der Verordnungen erlauben keinen Rückschluss auf eine mögliche Über-, Unter- oder Fehlversorgung.

\section{Neu 2019}

Gibt es eine Opioidepidemie in Deutschland? Evidenzbasierte Feststellung: Es gibt keine Hinweise auf eine Opioidepidemie in Deutschland. EL4 (100\%; $100 \%)$

Kommentar: Der Begriff „Opioidepidemie“ oder "Opioidkrise“ wurde für den parallelen Anstieg von Opioidverordnungen mit missbräuchlichem/ abhängigem Gebrauch der verordneten Opioide, Notfallaufnahmen in Krankenhäusern wegen Überdosierung und/ oder Todesfällen in den vergangenen 20 Jahren zunächst in den USA geprägt $[33,99]$. In den USA standen im Jahr 2014 Todesfälle bei 28.647 Personen und im Jahr 2017 bei 72.000 Menschen in Zusammenhang mit Opioiden. Die Todesrate lag 2016 bei 20 Drogentoten auf 100.000 Einwohner. Schätzungen gehen davon aus, dass $2 / 3$ der Drogentoten in den USA auf Opioide zurückzuführen sind $[27,28]$.

Die Organisation für wirtschaftliche Entwicklung und Zusammenarbeit (OECD) hat im Mai 2019 auf einen Anstieg opioidassoziierter Todesfälle in europäischen Ländern hingewiesen [111]. Die Bundesregierung hat auf eine Anfrage der FDP-Fraktion am 15.04.2019 geantwortet, dass es in Deutschland keine „Opioidkrise“ gibt wie in den USA. Die Verordnung und Abgabe stark wirksamer opioidhaltiger Arzneimittel sei in Deutschland im Vergleich zu den USA durch deutlich restriktivere Regelungen im Betäubungsmittelrecht bestimmt [41]. Die im Folgenden aufgeführten Daten unterstützen die Antwort der Bundesregierung.

In den USA wurden im Jahr 2016 bei $46 \%$ der Drogentoten synthetische Opioide (überwiegend illegales Heroin), bei ca. $40 \%$ rezeptpflichtige Opioide, bei $37 \%$ Heroin und bei $8 \%$ Methadon nachgewiesen. In den USA lag im Jahr 2015 die Rate von drogenbedingten notfallmäBigen Krankenhausaufnahmen bei 174 Aufnahmen auf 100.000 Einwohner, davon $25 \%$ wegen synthetischer Opioide, $15 \%$ wegen Heroin, 0,5\% wegen $\mathrm{Me}$ thadon und $10 \%$ wegen anderer Opioide $[28,134]$. Wie viele der Todesopfer bzw. Notfälle Opioide aus sinnvoller medizinischer Indikation erhielten, kann nicht eindeutig festgestellt werden. Ein- zelne Studien zeigen aber, dass bis zu $70 \%$ der Betroffenen zumindest einzelne Verordnungen erhielten [1]. Der Begriff "prescribed opioid crisis" ist aber dennoch irreführend, denn diese Krise entstand nicht nur, weil nach medizinischen Regeln behandelte Schmerzpatienten süchtig wurden. Vielmehr erkannten vermutlich bereits Süchtige und dafür Anfällige (die es auch unter Schmerzpatienten gibt) diese „legale“ Beschaffungsmethode für sich [98]. Vor allem Oxycodon wurde von einigen „pain doctors" großzügig an arbeitslose Weiße in einigen Bundesstaaten der Ostküste, die sich in einem ökonomischen Niedergang befanden, verschrieben [42]. Auch die Schmerzmedizin kann zur Opioidkrise durch die Propagierung von Schmerz als 5. Vitalzeichen [24], Anerkennung von US Krankenhäusern durch die Joint Commission nur im Falle der Zertifizierung für Schmerztherapie [9] und die ambulante Mitgabe bzw. Fortführung der Opioidtherapie auch nach einfachen operativen Eingriffen [107] beigetragen haben. Im US National Survey on Drug Use and Health berichteten $37,8 \%$ der befragten erwachsenen Personen im Jahr 2015, mindestens einmal in den letzten 12 Monaten verschreibungspflichtige Opioide eingenommen zu haben. 4,7\% der Befragten erfüllten die DSM-5-Kriterien eines Opioidmissbrauchs und 0,8\% einer „opioid use disorder“. Opioidmissbrauch und „opioid use disorder" wurden häufiger bei Personen nachgewiesen, die nicht krankenversichert und/ oder arbeitslos waren sowie ein niedriges Einkommen und psychische Probleme hatten. $60 \%$ berichteten, Opioide ohne Verschreibung einzunehmen [80]. Bis zu $90 \%$ der Heroinabhängigen in den USA konsumieren auch illegal erworbene verschreibungspflichtige Opioide [27].

Nach dem Arzneiverordnungsreport 2019 ist in Deutschland die Menge an "defined daily dosages“ (DDD) von Opioidanalgetika von 2009 bis 2016 langsam von 379 Mio. DDD auf 421 Mio. DDD gestiegen und blieb $2017 \mathrm{mit}$ 423 Mio. DDD und 2018 mit 422 Mio. DDD konstant. Das Verhältnis von schwachen und starken Opioiden ist mit etwa 60/40\% konstant [15]. Seit 20 Jahren ist die Anzahl Opiatabhängi- 
ger mit Kontakt zum Drogenhilfesystem konstant [92]. In Deutschland wurden im Jahr 20171272 Drogentote gezählt, entsprechend 2 Drogentoten auf 100.000 Einwohner. Die meisten Todesfälle waren auf Mischintoxikationen zurückzuführen. Am häufigsten waren Todesfälle mit Heroin/Morphin in Verbindung mit anderen Drogen (11\%) und Opiat- und Substitutionsmittel in Verbindung mit anderen Drogen (9\%). 2018 wurden 1276 Drogentote gezählt, davon $49 \%$ mit Heroin/Morphin. Wie viele der Todesopfer Opioide aus medizinischer Indikation erhielten, kann nicht eindeutig festgestellt werden [58]. Daten zu notfallmäßigen Krankenhausaufnahmen wegen Überdosierungen von Opioiden liegen aus Deutschland nicht vor. Bei einer Auswertung der Meldungen an die Nebenwirkungsdatenbank des Bundesinstituts für Arzneimittel und Medizinprodukte (BfArM) im Zeitraum 1978-2016 standen Opioide zusammengefasst mit Paracetamol, ASS und Metamizol mit 4,0\% der Meldungen auf Platz 7 der am häufigsten als verdächtigt gemeldeten Arzneimittelgruppen. Antipsychotika standen auf Rang 3, Antidepressiva auf Rang 4 und nicht-steroidale Antirheumatika (NSAR) auf Platz 5 [61]. Bei der Analyse von 10.174 konsekutiven Patienten aus vier deutschen Notaufnahmen erfolgten 6,5\% der Notfallaufnahmen wegen Nebenwirkungen von Medikamenten. 9\% der Notfallaufnahmen wegen Nebenwirkungen wurden auf die Einnahme von Opioiden zurückgeführt ( $12 \%$ auf peripher wirkende Analgetika, $11 \%$ auf Antidepressiva) [136]. Die Autoren des deutschen epidemiologischen Suchtsurvey folgern aus neun Erhebungen aus den Jahren 1995 bis 2018, dass in Deutschland keine „Opioidepidemie“ vergleichbar den USA vorliegt [139]. Diese Schlussfolgerung ist in Übereinstimmung mit der einer systematischen Übersichtsarbeit, welche 12 Studien aus Deutschland zu Opioidverschreibungen zusammengefasst hat [129].

\section{Neu 2019}

Prävalenz der missbräuchlichen/abhängigen Verwendung von aus medizinischer Indikation verschriebenen Opioiden. a) Evidenzbasierte Feststellungen: Die Prävalenz einer Gebrauchsstörung durch Opioidanalgetika nach Diagnostic and Statistical Manual for Psychiatric Diseases (DSM) 5 [4] liegt - basierend auf selbstberichteten Daten aus der allgemeinen Bevölkerungbei $1 \%$.

b) Die Prävalenzrate von Diagnosen von schädlichem Gebrauch/ Abhängigkeitssyndrom von aus medizinischer Indikation verschriebenen Opioiden - basierend auf Krankenkassendaten von stationären Aufenthalten in Deutschland - liegt bei Patienten mit einer Langzeitbehandlung mit Opioiden zwischen 0,8 und 1,8\%. EL3b. Konsens (75\%/78\%)

Kommentar: Es existieren nur wenige Studien zur Prävalenz- und Inzidenzschätzung aus Deutschland. Bei den Studien sind methodische und inhaltliche Limitationen $\mathrm{zu}$ berücksichtigen.

Der epidemiologische Suchtsurvey 2015 umfasste $n=9204$ Personen im Alter von 18 bis 64 Jahren aus der deutschen Bevölkerung (Antwortrate 52\%), die repräsentativ für die deutsche Bevölkerung waren. Die Befragung erfolgte direkt, telefonisch oder online. Für den Bereich der Schmerzmittel wurde das Vorliegen von Störungen nach DSM-5 in den letzten 12 Monaten mithilfe des Münchener Composite International Diagnostic Interview (M-CIDI) getrennt für opioidhaltige und nicht-opioidhaltige Schmerzmittel erfasst. Das Interview erfasst nicht, ob die Schmerzmittel von einem Arzt der interviewten Person aus medizinischer Indikation verschrieben wurden oder ob die interviewte Person die Schmerzmittel illegal erworben hat. Der epidemiologische Suchtsurvey (ESA) aus dem Jahr 2015 schätzte die Prävalenz einer Gebrauchsstörung durch Opioidanalgetika nach DSM-5 auf $1 \%$ $[6,7]$. Bei Telefoninterviews besteht das Risiko eines Verschweigens oder einer Dissimulation der missbräuchlichen Verwendung von aus medizinischer Indikation verschriebenen Opioiden [16].

In einer retrospektiven Querschnittsstudie im Jahr 2012 mit 800.000 Versicherten der Barmer Ersatzkasse lag die 1-Jahres-Prävalenz von Kranken- hausaufenthalten mit Diagnosen von psychischen und Verhaltensstörungen wegen Alkohol, Opioiden, Tranquilizern und multiplem Substanzgebrauch und Vergiftungen durch Betäubungsmittel bei $0,8 \%$ der Versicherten mit LTOT wegen chronischer nicht-tumorbedingter Schmerzen. Diese Diagnosen waren mit der Verschreibung von Tranquilizern (Odds Ratio [OR] 3,44; 95\%Konfidenzintervall [KI] 1,8-6,56) und mit Diagnosen depressiver Störungen (OR 2,26; 95\%-KI 1,37-6,58) und mit Diagnosen von somatoformer Schmerzstörung (OR 1,92; $95 \%$-KI 1,18-23,16) assoziiert. Es fand sich eine geringe negative Assoziation mit dem Alter (OR 0,96; 95\%-KI 0,95-0,98) [100].

In einer retrospektiven Querschnittsstudie im Jahr 2014 mit 4.028.618 Versicherten von 69 deutschen gesetzlichen Krankenversicherungen lag die 1-JahresPrävalenz der oben beschriebenen Krankenhausaufenthalte bei 1,75\% der Versicherten mit LTOT. Diese Diagnosen waren mit der Verschreibung von Tranquilizern (OR 3,63; $95 \%$-KI 3,03-4,36), mit Diagnosen depressiver Störungen (OR 2,52; $95 \%$-KI 2,12-3,00), mit Diagnosen von somatoformer Schmerzstörung (OR 1,89; $95 \%$-KI 1,56-2,28) und Hochdosis-LTOT (OR 1,81; $95 \%$-KI 1,44-2,27) assoziiert. Es fand sich eine negative Assoziation mit Alter 41-60 Jahre (OR 0,62; $95 \%$-KI 0,44-0,88), 61-80 Jahre (OR 0,30; $95 \%$-KI 0,21-0,42), >80 Jahre (OR 0,16; $95 \%$-KI $0,11-0,23$ ) und weiblichem Geschlecht (OR 0,47; $95 \%$ KI 0,40-0,55) [84]. Die Prävalenz von verordneten Opioiden, die mit Krankenhausaufenthalten aufgrund von Diagnosen von Missbrauch/Abhängigkeit zusammenhingen war für Tilidin/Naloxon $(43,3 \%)$, Tramadol $25,1 \%)$ und Oxycodon mit und ohne Naloxon (24,4\%) am höchsten, gefolgt von Fentanyl 16,4\%, Morphin 10,5\%, Hydromorphon 9,8\%, Buprenorphin 6,5\% und Tapentadol $4,4 \%$ [84]. Pharmakokinetisch ist ein höheres Risiko missbräuchlicher Verwendung von Oxycodon im Vergleich zu Morphin möglich, da $40 \%$ des retardierten Oxycodons schnell freigesetzt werden [121]. In einem deutschsprachigen Internetforum zum Thema Drogen in den Jahren 2010 und 2013 war Trama- 
dol das am häufigsten genannte Opioid für Missbrauch zu nicht-medizinischen Zwecken, gefolgt von Codein, Tilidin/ Naloxon, Morphin und Oxycodon [93].

Die Prävalenzraten beider Studien unterliegen Einschränkungen. Zunächst greifen die Datenbanken der gesetzlichen Krankenkassen auf Abrechnungsdaten $\mathrm{zu}$, die für die Abrechnung und nicht für Forschungszwecke erhoben wurden. Alle Analysen mit Routinedaten unterliegen möglichen Codierfehlern beziehungsweise „up-“ und „undercoding“. Ein „upcoding" liegt vor, wenn die Diagnose F 13.2 (Abhängigkeitssyndrom von Opioiden) in schmerzmedizinischen und psychiatrischen Einrichtungen bei Patienten nur aufgrund des Vorliegens einer körperlichen Abhängigkeit und/ oder Toleranz gestellt wird (siehe Kapitel „Missbrauch/schädlicher Gebrauch/ Abhängigkeitssyndrom“). Es wurden keine Interviews zu Verhaltensmustern von Missbrauch von verordneten Opioiden beziehungsweise dadurch bedingten Störungen geführt, was zu einer hohen Spezifität, aber geringen Sensitivität führen kann. Es sind Unterschätzungen der Prävalenzen möglich, wenn nur stationäre Daten Berücksichtigung gefunden haben und Untersuchungen zum illegalen Drogenkonsum nicht möglich waren [84].

\section{Fehlversorgung mit opioidhaltigen Analgetika in Deutschland}

Geprüft 2019. Evidenzbasierte Feststellung: Es liegen Hinweise für eine Fehlversorgung mit opioidhaltigen Analgetika bei einzelnen Krankheitsbildern in Deutschland vor. EL4. Starker Konsens

Kommentar ergänzt 2019: Studien, die eine Unterversorgung von CNTS in Deutschland belegen, sind den Autoren nicht bekannt. Es gibt weiterhin Hinweise für eine nicht-leitlinienkonforme Behandlung mit starken Opioiden bei Patienten mit funktionellen Störungen/ somatoformen Störungen in Deutschland [100]. 15,3\% der Versicherten mit einer LTOT wegen CNTS und einer Dosis von Morphinäquivalent von $>120 \mathrm{mg} / \mathrm{d}$ von 69 deutschen gesetzlichen Krankenkassen (standardisiert bzgl. Alter und Geschlecht auf die allgemeine deutsche
Bevölkerung) erhielten im Jahr 2014 die Diagnose einer somatoformen Schmerzstörung (F45.4x) [84]. Die S3-Leitlinie zu funktionellen Körperbeschwerden rät von einer Verordnung von Opioiden ab [128].

\section{Mögliche Indikationen und Kontraindikationen einer Behandlung mit opioidhaltigen Analgetika}

\section{Vorbemerkungen}

a) Die im Folgenden gebrauchte Formulierung „Therapieoption“ bedeutet, dass opioidhaltige Analgetika nur eine von verschiedenen medikamentösen Therapieoptionen sind.

b) Die Empfehlungen zur Dauer der Therapie (4-12 Wochen, 12-26 Wochen, $>26$ Wochen) bezieht sich laut den Einschlusskriterien der analysierten Studien auf Patienten mit chronischen (>3 Monate) bestehenden Schmerzen, die unter vorhergehenden medikamentösen und nichtmedikamentösen Therapien keine ausreichende Schmerzreduktion berichtet haben.

c) Die Stärke der Empfehlung für oder gegen opioidhaltige Analgetika impliziert keine Wertung gegenüber nicht-medikamentösen und anderen medikamentösen Therapien.

d) Die evidenz- und konsensbasierten Empfehlungen für einzelne Erkrankungen gelten für eine orale oder transdermale Therapie mit opioidhaltigen Analgetika.

e) Die Leitliniengruppe hat bei den verbalen Abstufungen des Empfehlungsgrades (soll, sollte, kann) eine weitere Differenzierung im Falle unzureichender Evidenz vorgenommen: Die Formulierung „kann empfohlen werden“ wird gewählt, wenn es eine positive Evidenz für eine kurzfristige, nicht jedoch für eine längerfristige Therapie gibt, jedoch auf der Basis eines klinischen Konsenses ein langfristiger individueller Therapieversuch bei Respondern möglich ist. Die Formulierung „kann erwogen werden“ wird gewählt, wenn es keine oder unzureichende Evidenz für einen kurzfristigen Therapieversuch gibt, jedoch auf der Basis eines klinischen Konsenses ein kurzfristiger und im Falle einer Therapieresponse - ein langfristiger Therapieversuch möglich ist. Der Begriff eines ,individuellen Heilversuchs" wird nicht verwendet, weil ein individueller Heilversuch als eine Behandlung außerhalb des Zulassungsbereichs definiert ist [87]. Eine kurz- und langfristige Therapie mit Opioiden ist jedoch innerhalb des Zulassungsbereichs der Opioide.

\section{Modifiziert 2019: chronischer Rückenschmerz (ICD-10 M42.16-M41.19, M42.90, M42.96-99, M43.0, M43.1, M47.26, M47.27, M47.29, M47.86, M47.87, 47.88, M47.99, M48.06, M48.2, M54.16, M54.5, M55.3, M.99.33; M99.43, M99.53)}

1.1 Konsensbasierte Empfehlung. Die kurz-, mittel- und langfristige Anwendung opioidhaltiger Analgetika soll auf Patienten mit einem nach ärztlich/ psychologisch/physiotherapeutischer Einschätzung relevanten somatischen Anteil in der Schmerzentstehung und -aufrechterhaltung und unzureichendem Ansprechen auf nicht-medikamentöse Therapien beschränkt werden. Starke Empfehlung (100\%; $100 \%)$

Kommentar: Die konsensbasierte Empfehlung erfolgte, um die in Anbetracht der Häufigkeit chronischer Rückenschmerzen potenzielle Zahl möglicher Patienten auf solche zu reduzieren, bei denen nach Ansicht der Leitliniengruppe Opioide eine Therapieoption darstellen wie bei Patienten mit chronisch-entzündlichen Rückenerkrankungen oder inoperablen Spinalkanalstenosen. Die Leitliniengruppe ist der Ansicht, dass Patienten mit funktionellen Rückenschmerzen oder psychischen Störungen mit dem Leitsymptom chronischer Rückenschmerz nicht mit Opioiden behandelt werden sollen.

\subsection{Evidenzbasierte Empfehlung.}

12 Wochen: Opioidhaltige Analgetika sollten Patienten mit chronischem Rückenschmerz als eine Therapieoption empfohlen werden. EL1a, Empfehlung**. Starker Konsens (100\%; 100\%) 


\begin{tabular}{|c|c|c|c|c|}
\hline $\begin{array}{l}\text { Studiendauer (für RCT } \\
\text { Dauer doppelblinde } \\
\text { Phase) }\end{array}$ & 4-12 Wochen [118] & $\begin{array}{l}13-26 \text { Wo- } \\
\text { chen [118] }\end{array}$ & 27-52 Wochen [12] & $>52$ Wochen $[2]$ \\
\hline $\begin{array}{l}\text { Anzahl Studien mit } \\
\text { einem Parallel- und } \\
\text { Cross-over-Design und } \\
\text { Patienten }\end{array}$ & $5 / 1500$ & $4 / 1936$ & $4 / 486$ & 1/680 (beendet 341) \\
\hline Evidenzlevel & la & la & $\begin{array}{l}\text { 2a (SR von Open-label-Stu- } \\
\text { dien) }\end{array}$ & 1b (randomisiert "open label“, kein Placebo) \\
\hline $\begin{array}{l}\text { Schmerzreduktion } 50 \% \\
\text { und mehr }\end{array}$ & $\begin{array}{l}\operatorname{RD} 0,07(0,01 ; 0,13) \\
\left(I^{2}=0 \%, p=0,01\right) \\
\text { NNTB } 16(7,100)\end{array}$ & $\begin{array}{l}\operatorname{RD} 0,09(0,03 ; 0,14) \\
\left(I^{2}=0 \%, p=0,002\right) \\
\text { NNTB } 11(7,33)\end{array}$ & $\begin{array}{l}\text { Keine signifikante Änderung } \\
\text { Schmerzintensität in open } \\
\text { label: SMD } 0,34(-0,17 ; 0,86)\end{array}$ & $\begin{array}{l}\geq 50 \% \text { Reduktion Ruheschmerz: } 40 \% \text { durch } \\
\text { Fentanyl, } 37 \% \text { durch Morphin } \\
\geq 50 \% \text { Reduktion Bewegungsschmerz: } 47 \% \\
\text { durch Fentanyl, } 50 \% \text { durch Morphin }\end{array}$ \\
\hline $\begin{array}{l}\text { Starke oder sehr starke } \\
\text { globale Besserung }\end{array}$ & $\begin{array}{l}\operatorname{RD} 0,12(-0,03 ; 0,27) \\
\left(I^{2}=84, p=0,11\right)\end{array}$ & $\begin{array}{l}\operatorname{RD} 0,19(0,12 ; 0,26) \\
\left(I^{2}=0, p<0,0001\right) \\
\text { NNTB } 5(4,6)\end{array}$ & Keine Daten & Keine Daten \\
\hline $\begin{array}{l}\text { Beeinträchtigungs- } \\
\text { erleben }\end{array}$ & $\begin{array}{l}\text { SMD }-0,21(-0,33 \\
-0,09) ; I^{2}=0 \% \\
p<0,0009\end{array}$ & $\begin{array}{l}\operatorname{SMD}-0,23(-0,34 \\
-0,11) ; I^{2}=0 \\
p<0,0001\end{array}$ & Keine ausreichenden Daten & $\begin{array}{l}\text { Statistisch signifikante Prä-post-Verbesse- } \\
\text { rung in beiden Gruppen }\end{array}$ \\
\hline $\begin{array}{l}\text { Abbruchraten wegen } \\
\text { Nebenwirkungen }\end{array}$ & $\begin{array}{l}\text { RD } 0,04(-0,01 ; 0,10) \\
\left(I^{2}=62 \% ; p=0,13\right) \\
\text { NNTH } 25(10,100)\end{array}$ & $\begin{array}{l}\text { RD } 0,21(0,14 ; 0,27) \\
\left(I^{2}=78 \% ; p<0,001\right) \\
\text { NNTH } 5(4,7)\end{array}$ & $3,8 \%$ & $\begin{array}{l}37 \% \text { in Fentanyl- und } 31 \% \text { in Morphingrup- } \\
\text { pe }\end{array}$ \\
\hline $\begin{array}{l}\text { Häufigkeit schwerer } \\
\text { Nebenwirkungen }\end{array}$ & $\begin{array}{l}\text { Keine signifikanten } \\
\text { Unterschiede }\end{array}$ & $\begin{array}{l}\text { Keine signifikanten } \\
\text { Unterschiede }\end{array}$ & $4,8 \%$ & Keine berichtet \\
\hline
\end{tabular}

\subsection{Evidenzbasierte Empfehlung.}

13-26 Wochen: Opioidhaltige Analgetika sollten Patienten mit chronischem Rückenschmerz als eine Therapieoption empfohlen werden. EL1a, Empfehlung**. Starker Konsens (100\%; 100\%). (** Grund der Abwertung des Empfehlungsgrades um eine Stufe: höhere Risiken im Vergleich zu nicht-medikamentösen Therapien)

\subsection{Evidenzbasierte Empfehlung.}

>26 Wochen: Opioidhaltige Analgetika können Patienten mit chronischem Rückenschmerz als eine Therapieoption empfohlen werden. EL2b, offene Empfehlung. Starker Konsens (100\%; 100\%)

Kommentar: Es wurde eine Literatursuche nach neuen RCTs in CENTRAL, PubMed und PsycINFO von Oktober 2013 bis Juni 2019 durchgeführt. Es wurden 9 neue RCTs mit 2980 Patienten gefunden. Für die Metaanalyse wurden 8 RCTs mit 9 Studienarmen und 3436 Teilnehmern mit einem Parallelund Cross-over-Design und 12 Studien mit einem EERW-Design und 4112 Patienten in die qualitative und quan- titative Analyse eingeschlossen [118]. Weiterhin wurden vier neue „open label extension trials“ analysiert [12]. Die eingesetzten Opioide waren Buprenorphin, Hydromorphon, Morphin, Oxycodon, Tapentadol und Tramadol. Anhand der Ein- und Ausschlusskriterien ließ sich keine Zuordnung zu spezifischen und nicht-spezifischen Rückenschmerzen in den meisten Studien durchführen. Da Studien mit einem EERW-Design die Verträglichkeit von Medikamenten überschätzen (es werden nur Patienten in die doppelblinde Phase eingeschlossen, welche das Medikament tolerieren und eine Schmerzreduktion angeben) [74], stützen sich die Empfehlungen auf die Studien mit einem Parallel- und Crossover-Design. In kurz- und mittelfristigen Studien fanden sich eine klinisch nicht relevante Überlegenheit gegenüber Placebo in Schmerzreduktion 50\% und mehr und keine Unterschiede zu Placebo in der Häufigkeit schwerer Nebenwirkungen. In kurzfristigen Studien fanden sich eine klinisch relevante Reduktion des Beeinträchtigungserlebens und kein Unterschied zu Placebo in der globalen
Verbesserung. Die höhere Abbruchrate wegen Nebenwirkungen war in kurzfristigen Studien nicht und in mittelfristigen Studien klinisch relevant.

Langfristige Studien konnten in Form von 4 „open label extension trials“ mit 486 Patienten und einer 1-jährigen offenen Studie mit Direktvergleich orales Morphin versus transdermales Fentanyl [2] analysiert werden. In den „open label extension studies“ fand sich keine Änderung der durchschnittlichen Schmerzintensität. In einer offenen, kontrollierten Studie erhielten 675 Patienten mit chronischem Rückenschmerz (nozizeptiv, neuropathisch, gemischt nozizeptiv/ neuropathisch) über 13 Monate transdermales Fentanyl oder orales Morphin. $37 \%$ (40\%) der Patienten in der Fentanylgruppe und $37 \%$ (50\%) der Patienten in der Morphingruppe gaben am Therapieende eine 50 \%ige Reduktion der Schmerzen in Ruhe (in Bewegung) an. Die körperliche Funktionsfähigkeit („SF36 physical functioning “) verbesserte sich im Durchschnitt signifikant $(p<0,0001)$ von 29 auf 37 (Skala 0 [schlechtester Wert] - 100 [bester Wert] in beiden Gruppen. 
Tab. 2 Wirksamkeit, Verträglichkeit und Sicherheit von Opioiden bei chronischen Arthroseschmerzen. (Modifiziert nach [12, 155])

\begin{tabular}{|c|c|c|c|}
\hline $\begin{array}{l}\text { Studiendauer (für RCT Dauer } \\
\text { doppelblinde Phase) }\end{array}$ & 4-12 Wochen & 13-26 Wochen & 27-78 Wochen \\
\hline Anzahl RCTs und Patienten & $12 / 4372$ & $6 / 3577$ & $\begin{array}{l}4 \text { „open label exten- } \\
\text { sion"/448 }\end{array}$ \\
\hline Evidenzlevel & $1 a$ & $1 \mathrm{a}$ & $\begin{array}{l}\text { 2a (SR von Open-la- } \\
\text { bel-Studien) }\end{array}$ \\
\hline $\begin{array}{l}\text { Schmerzreduktion } 50 \% \text { und } \\
\text { mehr }\end{array}$ & $\begin{array}{l}12 / 4219: \operatorname{RD} 0,04(0,01 ; 0,07) ; I^{2}=46 ; \\
p=0,006 \\
\text { NNTB } 25(16,100)\end{array}$ & $6 / 3476: \mathrm{RD}-0,01(0,05 ; 0,03) ; 1^{3}=46 ; p=0,66$ & $\begin{array}{l}\text { Keine ausreichenden } \\
\text { Daten }\end{array}$ \\
\hline $\begin{array}{l}\text { Reduktion Beeinträchtigungs- } \\
\text { erleben }\end{array}$ & $\begin{array}{l}11 / 3864: \text { SMD }-0,23(-0,30 ;-0,16) \\
\mathrm{I}^{2}=88 \% ; p<0,0001\end{array}$ & $\begin{array}{l}4 / 2121: \text { SMD }-0,23(-0,30 ; 0,03) ; I^{2}=69 ; \\
p=0,11\end{array}$ & $\begin{array}{l}\text { Keine ausreichenden } \\
\text { Daten }\end{array}$ \\
\hline $\begin{array}{l}\text { Starke oder sehr starke globale } \\
\text { Besserung }\end{array}$ & Keine Daten & $\begin{array}{l}3 / 2209: \operatorname{RD} 0,07(0,00 ; 0,14)\left(I^{2}=61 \%, p=0,04\right) \\
\text { NNTB } 16(7,100)\end{array}$ & $\begin{array}{l}\text { Keine ausreichenden } \\
\text { Daten }\end{array}$ \\
\hline $\begin{array}{l}\text { Abbruchraten wegen Neben- } \\
\text { wirkungen }\end{array}$ & $\begin{array}{l}\text { 12/4372: RD 0,17 }(0,13 ; 0,21) ; I^{2}=72 ; \\
p<0,001 \\
\text { NNTH } 6(5,7)\end{array}$ & $\begin{array}{l}6 / 3577 ; \operatorname{RD~} 0,24(0,17 ; 0,31) ; I^{2}=88 ; p<0,0001 \\
\text { NNTH } 4(3,6)\end{array}$ & $29,9 \%$ \\
\hline $\begin{array}{l}\text { Häufigkeit schwerer Nebenwir- } \\
\text { kungen }\end{array}$ & $\begin{array}{l}7 / 2585: \mathrm{RD}-0,00(-0,01 ; 0,01) ; I^{2}=0 ; \\
p=0,55\end{array}$ & $\begin{array}{l}\text { 5/3160; RD } 0,01(0,00 ; 0,02) ; l^{2}=0 ; p=0,003 \\
\text { NNTH } 100 \text { ( } 50 \text { bis unendlich) }\end{array}$ & $5,1 \%$ \\
\hline
\end{tabular}

Die Abbruchrate lag bei $37 \%$ bei Fentanyl und $31 \%$ bei Morphin. Todesfälle oder süchtiges Verhalten wurden nicht beobachtet ([2]; - Tab. 1).

Laut der Nationalen Versorgungsleitlinie „Nicht-spezifischer Kreuzschmerz“ können Opioide zur Behandlung chronischer nicht-spezifischer Kreuzschmerzen auch als langfristige Therapieoption angewendet werden, wenn unter einer zeitlich befristeten Therapie (vier bis zwölf Wochen) eine klinisch relevante Reduktion der Schmerzen und/oder des körperlichen Beeinträchtigungserlebens bei fehlenden oder geringen Nebenwirkungen eingetreten ist. Opioide sollen zur Langzeitbehandlung chronischer nicht-spezifischer Kreuzschmerzen nur im Rahmen eines therapeutischen Gesamtkonzepts angewendet werden [31].

Die S2k-Leitlinie zum spezifischen Kreuzschmerz macht keine Aussage über Opioide [49].

Eine Übersicht über internationale Leitlinien zum chronischen Rückenschmerz in der Primärversorgung stellte fest, dass sich die meisten Leitlinien auf sogenannte nicht-spezifische Rückenschmerzen beziehen. Die Mehrzahl der Leitlinien empfiehlt als Erstlinientherapie nicht-medikamentöse Therapieverfahren und Opioide nur bei ausgewählten Patienten im Falle des Versagens nichtmedikamentöser Therapien [114].

\section{Modifiziert 2019: chronischer} Arthroseschmerz (ICD-10 M15-19) 2.1 Konsensbasierte Empfehlung. Die Anwendung opioidhaltiger Analgetika bei Patienten mit Arthroseschmerzen soll auf folgende klinische Konstellationen beschränkt werden: Versagen nicht-medikamentöser Therapien; Wirkungslosigkeit bzw. Kontraindikationen anderer Analgetika; Gelenkersatz nicht möglich oder von Patienten nicht gewünscht. Starke Empfehlung. Starker Konsens (100\%; $100 \%)$

\subsection{Evidenzbasierte Empfehlung.}

4-12 Wochen: Opioidhaltige Analgetika sollten Patienten mit Arthroseschmerzen als eine Therapieoption empfohlen werden. EL1a, Empfehlung . Konsens (78\%; $75 \%)$. ( ${ }^{*}$ Grund der Abwertung des Empfehlungsgrades um eine Stufe: höhere Risiken im Vergleich zu nicht-medikamentösen Therapien)

\subsection{Evidenzbasierte Empfehlung.}

13-26 Wochen: Opioidhaltige Analgetika sollten Patienten mit Arthroseschmerzen als eine Therapieoption empfohlen werden. EL1a, Empfehlung*. Konsens (83\%; $81 \%$ )

\subsection{Evidenzbasierte Empfehlung.}

>26 Wochen: Opioidhaltige Analgetika können Patienten mit Arthroseschmerzen als eine Therapieoption empfohlen wer- den. EL2b, offene Empfehlung. Starker Konsens (100\%; 100\%)

Kommentar: Es wurde eine Literatursuche nach neuen RCTs in CENTRAL, PubMed und PsycINFO von Oktober 2013 bis Juni 2019 durchgeführt. Es wurden 8 neue RCTs mit 2728 Patienten in die Metaanalysen eingeschlossen. Zur Erstellung der Empfehlungen wurden die Ergebnisse von 20 RCTs mit 7398 Patienten und den Opioiden Buprenorphin, Fentanyl, Hydrocodon, Hydromorphon, Morphin, Oxymorphon, Tapentadol und Tramadol in die qualitative und quantitative Analyse eingeschlossen. Die Indikationen waren Knie- und/oder Hüftschmerzen [155]. Weiterhin wurden vier neue „open label extension studies“ analysiert [12]. In kurzfristigen Studien war die Überlegenheit gegenüber Placebo in der $50 \%$ igen und größeren Schmerzreduktion nicht klinisch relevant, jedoch die Reduktion des Beeinträchtigungserlebens. Die höhere Abbruchrate wegen Nebenwirkungen war klinisch relevant. In mittelfristigen Studien zeigte sich keine Überlegenheit gegenüber Placebo in der $50 \%$ igen und größeren Schmerzreduktion und dem Beeinträchtigungserleben, jedoch eine klinisch nicht relevante globale Besserung. Die höhere Abbruchrate wegen Nebenwirkungen war klinisch relevant. Die Daten bezüglich Wirksamkeit von vier „open label extension studies" waren nicht für eine quantitative Analyse geeignet (• Tab. 2). 


\begin{tabular}{|c|c|c|c|}
\hline Studiendauer (für RCT Dauer doppelblinde Phase) & 4-12 Wochen & 13-26 Wochen & $>26$ Wochen \\
\hline Anzahl Studien und Patienten & $7 / 1098$ & Keine RCT & $1 / 117$ \\
\hline Evidenzlevel & la & - & $2 b_{\text {„open label extension" }}$ \\
\hline Schmerzreduktion $50 \%$ und mehr & $\begin{array}{l}2 \text { RCTs }^{\mathrm{a}} \text { mit EERW-Design } \\
\text { RD } 0,11(0,04 ; 0,18) \\
\text { NNTB } 9(6,25)\end{array}$ & - & $\begin{array}{l}\text { Keine signifikante Ände- } \\
\text { rung der Schmerzintensität }\end{array}$ \\
\hline Starke oder sehr starke globale Besserung & $\begin{array}{l}2 \text { RCTs }^{\mathrm{a}} \text { mit EERW-Design } \\
\text { RD } 0,24(0,16 ; 0,31) \\
\text { NNTB } 4(3,6)\end{array}$ & - & Keine Daten \\
\hline Reduktion Beeinträchtigungserleben & $\begin{array}{l}1 \mathrm{RCT}^{\mathrm{a}} \text { mit ERRW-Design } \\
\text { SMD }-0,36(-0,60 ;-0,12)\end{array}$ & - & Keine Daten \\
\hline Abbruchraten wegen Nebenwirkungen & $\begin{array}{l}2 \text { RCTs }^{\mathrm{a}} \text { mit EERW-Design } \\
\text { RD } 0,06(0,02 ; 0,11) \\
\text { NNTH } 16(9,50)\end{array}$ & - & $11 \%$ \\
\hline Häufigkeit schwerer Nebenwirkungen & $\begin{array}{l}2 \text { RCTs }^{\mathrm{a}} \text { mit EERW-Design } \\
\mathrm{RD} 0,02(-0,03 ; 0,06)\end{array}$ & - & $8,5 \%$ \\
\hline
\end{tabular}

Eine systematische Übersicht mit RCTs von mindestens 8 Wochen Dauer schloss 27 Behandlungsarme mit NSAIDs und 14 Behandlungsarme mit Opioiden ein und fand keine Unterschiede in der Schmerzreduktion [143].

Eine Übersicht über Arthroseleitlinien (Literatursuche bis April 2013) fand 16 Leitlinien. Die meisten Leitlinien gaben starke Empfehlungen für nichtmedikamentöse Behandlungen (Trainingstherapie; Gewichtsabnahme bei Übergewicht) und bei geeigneten Patienten für einen Kniegelenkersatz. Die empfohlene medikamentöse Erstlinientherapie mit Paracetamol kann nicht durch aktuelle Metaanalysen gestützt werden [37]. Empfohlene Zweitlinientherapien waren topisches Capsaicin und NSAR sowie orale NSAR. Für refraktäre Schmerzen wurde Tramadol empfohlen. Andere Opioide oder Duloxetin können erwogen werden [106]. In der klinischen Praxis ist eine Therapie mit NSAR häufig aufgrund der Begleiterkrankungen des Patienten (z.B. kardiovaskuläre Erkrankungen, Niereninsuffizienz, Ulkusanamnese) kontraindiziert.

Die S2k-Leitlinie zur Koxarthrose empfiehlt, dass Opioide dann zum Einsatz kommen sollen, wenn andere Maßnahmen wie die Gabe von NSAR oder operative Maßnahmen nicht möglich sind oder andere Komorbiditäten keine andere Wahl zulassen [48]. Die S2kLeitlinie zur Gonarthrose stellt fest, dass der kurzfristige Einsatz von schwachen Opioiden bei nicht-operablen Patienten oder bei Patienten, die für kurze Zeit bis zu einer Operation begleitet werden, sinnvoll sein kann [50].

\section{Modifiziert 2019: diabetische Polyneuropathie (ICD-10 G63.2*) 3.1 Evidenzbasierte Empfehlung.}

4-12 Wochen: Opioidhaltige Analgetika sollen Patienten mit diabetischer Polyneuropathie als eine Therapieoption empfohlen werden. EL1a, starke Empfehlung. Starker Konsens (100\%; $100 \%)$

\subsection{Evidenzbasierte Empfehlung.}

13-26 Wochen: Opioidhaltige Analgetika können Patienten mit diabetischer Polyneuropathie als eine Therapieoption empfohlen werden. EL2b, offene Empfehlung. Starker Konsens (100\%; $100 \%)$

\subsection{Evidenzbasierte Empfehlung.} >26 Wochen: Opioidhaltige Analgetika können Patienten mit diabetischer Polyneuropathie als eine Therapieoption empfohlen werden. EL2b, offene Empfehlung. Starker Konsens (100\%; $100 \%)$

Kommentar: Für alle neuropathischen Schmerzen wurde eine Literatursuche nach neuen RCTs in CENTRAL, PubMed und PsycINFO von Oktober 2013 bis Juni 2019 durchgeführt. 4 neue RCTs mit 662 Patienten wurden in die Metaanalyse eingeschlossen [145]. Insgesamt wurden 16 RCTs mit 2292 Patienten analysiert. Weiterhin wurde eine neue "open label extension study“ analysiert [12].

Für die diabetische Polyneuropathie wurden 7 RCTs mit Oxycodon, Tapentadol und Tramadol in die quantitative und qualitative Analyse eingeschlossen [145]. Bezüglich der Reduktion der Schmerzintensität von $50 \%$ und mehr und des Beeinträchtigungserlebens sowie der starken oder sehr starken globalen Symptomverbesserung waren die Effekte klinisch relevant. Die erhöhten Abbruchraten wegen Nebenwirkungen warenklinisch nicht relevant. Die Häufigkeit schwerer Nebenwirkungen unterschied sich nicht von Placebo (- Tab. 3).

Die Nationale Versorgungsleitlinie (NVL) „Neuropathie bei Diabetes im Erwachsenenalter“ führt aus: „Keine Studie belegt, dass bestimmte als analgetisch wirksam bewiesene Substanzen Mittel der ersten Wahl sind. Es existieren für die Behandlung neuropathischer Schmerzen Therapiealgorithmen. In den meisten Leitlinien wird empfohlen, Antidepressiva oder Antikonvulsiva vorrangig einzusetzen, und Opioide erst dann, wenn die vorgenannten Substanzgruppen, allein oder in Kombination gegeben, versagt haben. ... Davon abweichend kann auch ein Opioid als Schmerzmittel der ersten Wahl gegeben werden, wenn eine Komorbidität (z.B. Herzrhythmusstörungen, deutliches Übergewicht) gegen den primären 
Tab. 4 Wirksamkeit, Verträglichkeit und Sicherheit von Opioiden bei chronischen Schmerzen bei Postzosterneuralgie. (Modifiziert nach [145])

Studiendauer (für RCT Dauer doppelblinde Phase)

Anzahl RCTs und Patienten
4-12 Wochen

$3 / 323$
13-26 Wochen

Keine kontrollierte Studie
$>26$ Wochen

Keine kontrollierte Studie Keine Open-label-extension-Studie

\section{Evidenzlevel}

Schmerzreduktion

Starke oder sehr starke globale Besserung

Reduktion Beeinträchtigungserleben

Abbruchraten wegen Nebenwirkungen

Häufigkeit schwerer Nebenwirkungen la

SMD $-0,49(-0,75 ;-0,28)$

Keine ausreichende Daten

Keine ausreichende Daten

$\operatorname{RD} 0,08(0,04 ; 0,13)$

NNTH $12(8,25)$

$\operatorname{RD} 0,10(-0,10 ; 0,12)$
$-$
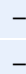

Einsatz anderer (Ko-)Analgetika spricht, die wie TZA (trizyklische Antidepressiva) oder Pregabalin zur Gewichtszunahme oder zu Arrhythmien führen können“. An gleichberechtigten medikamentösen Therapieoptionen gibt die NVL eine Empfehlung für trizyklische Antidepressiva, Duloxetin und Pregabalin. An opioidhaltigen Analgetika, für die eine Evidenz durch kontrollierte Studien vorliegt, werden Methadon, Morphin, Oxycodon und Tramadol genannt [19].

\section{Modifiziert 2019: Postzoster- neuralgie (ICD-10 B02.2+)}

\subsection{Evidenzbasierte Empfehlung.}

4-12 Wochen: Opioidhaltige Analgetika sollten Patienten mit Postzosterneuralgie als eine Therapieoption angeboten werden. EL1a, Empfehlung*. Starker Konsens (100\%; $100 \%)$. ( Erläuterung der Gründe der Abwertung des Empfehlungsgrades um 1 Stufe: Anzahl Patienten <400 in Metaanalyse.)

\subsection{Konsensbasierte Empfehlung.}

13-26 Wochen: Opioidhaltige Analgetika können Patienten mit Postzosterneuralgie mit Therapieresponse als eine Therapieoption empfohlen werden. Offene Empfehlung. Starker Konsens (100\%; 100\%)

\subsection{Konsensbasierte Empfehlung.}

>26 Wochen: Opioidhaltige Analgetika können Patienten mit Postzosterneuralgie mit Therapieresponse als eine Therapieoption empfohlen werden. Offene Empfehlung. Starker Konsens (100\%; $100 \%)$

Kommentar: Es liegen 3 RCTs mit Parallel- bzw. Cross-over-Design (Morphin Oxycodon, Tramadol) und 323 Patienten mit einem Median der Studiendauer von
6 (4-8) Wochen vor. Opioidhaltige Analgetika waren Placebo in der Reduktion von Schmerzen klinisch relevant überlegen und Placebo in der Verträglichkeit unterlegen. Die erhöhten Abbruchraten wegen Nebenwirkungen waren klinisch nicht relevant ([145]; • Tab. 4).

Es liegt keine deutsche S3-Leitlinie zur Postzosterneuralgie (PZN) vor. In einer Übersichtsarbeit zu Leitlinien der PZN wurden als Erstlinienmedikamente trizyklische Antidepressiva, Gabapentin und Pregabalin sowie Lidocain-5\%-Pflaster genannt. Opioide inkl. Tramadol, Capsaicincreme und -pflaster wurden als Zweitlinientherapieoptionen genannt [81].

\section{Modifiziert 2019: Phantom- schmerz (ICD-10 G54.6)}

5.1 Evidenzbasierte Empfehlung.

4-12 Wochen: Opioidhaltige Analgetika sollten Patienten mit Phantomschmerz als eine Therapieoption angeboten werden. EL1b, Empfehlung*. Starker Konsens (100\%; 100\%). ( ( Gründe der Abwertung des Empfehlungsgrades um 1 Stufe: Anzahl Patienten $<400$ in Metaanalyse)

\subsection{Konsensbasierte Empfehlung.}

13-26 Wochen: Opioidhaltige Analgetika können Patienten mit Phantomschmerz mit Therapieresponse als eine Therapieoption empfohlen werden. Offene Empfehlung. Starker Konsens (100\%; $100 \%)$

\subsection{Konsensbasierte Empfehlung.}

>26 Wochen: Opioidhaltige Analgetika können bei Patienten mit Phantomschmerz und Therapieresponse als eine Therapieoption empfohlen werden. Offene Empfehlung. Starker Konsens (100\%; $100 \%)$
Kommentar: Es liegt eine RCT mit Morphin mit 60 Patienten über 8 Wochen vor. Morphin war Placebo nicht bezüglich der $50 \%$ und mehr Schmerzreduktion, aber in der durchschnittlichen Schmerzreduktion klinisch relevant überlegen. Die übrigen Ergebnisvariablen wurden unvollständig berichtet ([145]; • Tab. 5).

Es liegt keine deutsche S3-Leitlinie zum Phantomschmerz vor.

\section{Modifiziert 2019: Schmerz nach Rückenmarksverletzung 6.1 Evidenzbasierte Empfehlung.}

4-12 Wochen: Opioidhaltige Analgetika sollten Patienten mit Schmerzen nach Rückenmarksverletzung nicht als eine primäre Therapieoption empfohlen werden. EL1b, Empfehlung**. Starker Konsens (94\%; 94\%). ( ${ }^{\star}$ Gründe der Abwertung des Empfehlungsgrades: nur 1 RCT; ${ }^{* *}$ „Sollte nicht $"=$,kann einigen Personen angeboten werden“)

\subsection{Konsensbasierte Empfehlung.}

13-26 Wochen: Opioidhaltige Analgetika können Patienten mit Schmerzen nach Rückenmarksverletzung als individueller Therapieversuch bei Therapieresponse als eine Therapieoption angeboten werden. Offene Empfehlung. Starker Konsens (100\%; 100\%)

\subsection{Konsensbasierte Empfehlung.}

>26 Wochen: Opioidhaltige Analgetika können Patienten mit Schmerzen nach Rückenmarksverletzung als individueller Therapieversuch bei Therapieresponse als eine Therapieoption angeboten werden. Offene Empfehlung 
Tab. 5 Wirksamkeit, Verträglichkeit und Sicherheit von Opioiden bei chronischen Phantomschmerzen. (Modifiziert nach [145])

\begin{tabular}{|c|c|c|c|}
\hline Dauer doppelblinde Phase & 4-12 Wochen & 13-26 Wochen & $>26$ Wochen \\
\hline Anzahl Studien und Patienten & $1 / 60$ & Keine kontrollierte Studie & $\begin{array}{l}\text { Keine kontrollierte Studie } \\
\text { Keine Open-label-exten- } \\
\text { sion-Studie }\end{array}$ \\
\hline Evidenzlevel & $\mathrm{lb}$ & - & - \\
\hline Schmerzreduktion $50 \%$ und mehr & RD $0,16(-0,04 ; 0,35)$ & - & - \\
\hline Durchschnittliche Schmerzreduktion & SMD $-0,95(-1.01 ;-0,17)$ & - & - \\
\hline Starke oder sehr starke globale Besserung & Keine Daten & - & - \\
\hline Reduktion Beeinträchtigungserleben & Keine Daten & - & - \\
\hline Abbruchraten wegen Nebenwirkungen & Keine Daten & - & - \\
\hline Häufigkeit schwerer Nebenwirkungen & Keine Daten & - & - \\
\hline
\end{tabular}

Tab. 6 Wirksamkeit, Verträglichkeit und Sicherheit von Opioiden bei chronischen Schmerzen nach Rückenmarksverletzung. (Modifiziert nach [145])

Dauer doppelblinde Phase

Anzahl Studien und Patienten

Evidenzlevel

Schmerzreduktion $50 \%$ und mehr

Starke oder sehr starke globale Besserung

Reduktion Beeinträchtigungserleben

Abbruchraten wegen Nebenwirkungen

Häufigkeit schwerer Nebenwirkungen

$4-12$ Wochen
$1 / 35$

$\mathrm{Ib}$

$\operatorname{RD} 0,17(-0,01 ; 0,36)$

RD $0,17(-0,01 ; 0,36)$

SMD $-0,68(-1.40 ; 0,03)$

$\operatorname{RD} 0,31(0,02 ; 0,61)$

NNTH $3(2,50)$

Keine Daten

\section{3-26 Wochen \\ Keine kontrollierte Studie}

$>26$ Wochen

Keine kontrollierte Studie Keine Open-label-extension-Studie
Kommentar: Es liegt eine RCT mit Tramadol bei 35 Patienten und einer Studiendauer von 6 Wochen vor. Tramadol war Placebo bezüglich Effektivität nicht überlegen. Die erhöhte Abbruchrate wegen Nebenwirkungen war klinisch relevant (• Tab. 6).

Es liegt eine offene Beobachtungsstudie ( $n=54$ Patienten) mit Oxycodon vor, die bei einer Kombination mit Antikonvulsiva eine Abnahme der Schmerzintensität berichtete [11].

In der S2k-LL zu Schmerzen bei Querschnittslähmung wird für nozizeptive (z.B. myofasziale) Schmerzen ein sehr zurückhaltender Einsatz empfohlen, da kein Wirksamkeitsnachweis gesehen wird, für neuropathischen Schmerz wurden Hinweise für eine Wirkung gesehen. Grundsätzlich bestand ein starker Konsens für eine offene Empfehlung für die Gabe von Opioiden (Oxycodon/ Tramadol) in „Güterabwägung mit dem Nebenwirkungsprofil“ bei nozizeptivem Schmerz und neuropathischem Schmerz (Tramadol). Für Oxycodon wurde eine offene Empfehlung für die Kombination mit Antikonvulsiva formuliert, eine
Monotherapie aber als ,sollte nicht empfohlen werden“ eingestuft [54].

Trotz der negativen RCT erfolgte eine offene konsensbasierte Empfehlung für den längerfristigen Einsatz bei Therapierespondern, weil alle bei der Leitlinienkonferenz anwesenden Neurologen und Orthopäden, die Patienten mit Rückenmarksverletzungen behandeln, sowohl auf die unzureichende Evidenz der Wirksamkeit medikamentöser Alternativen als auch auf eigene positive Erfahrungen mit Opioiden bei einzelnen Patienten hinwiesen.

\section{Modifiziert 2019: schmerzhafte Radikulopathie (ICD-10 M54.1) 7.1 Evidenzbasierte Empfehlung.}

4-12 Wochen: Opioidhaltige Analgetika können Patienten mit schmerzhafter Radikulopathie als individueller Therapieversuch angeboten werden. EL1b, offene Empfehlung*. Starker Konsens (100\%; $100 \%)$. ( ${ }^{\star}$ Gründe der Abwertung um eine Stufe: inkonsistente Studienergebnisse)

\subsection{Konsensbasierte Empfehlung.}

13-26 Wochen: Opioidhaltige Analgetika können Patienten mit schmerzhafter Radikulopathie als individueller Therapieversuch bei Therapieresponse als eine Therapieoption angeboten werden. Offene Empfehlung. Starker Konsens (100\%; $100 \%)$

\subsection{Konsensbasierte Empfehlung.}

>26 Wochen: Opioidhaltige Analgetika können Patienten mit schmerzhafter Radikulopathie als individueller Therapieversuch bei Therapieresponse als eine Therapieoption angeboten werden. Offene Empfehlung. Starker Konsens (100\%; $100 \%)$

Kommentar: Es liegen zwei RCTs zu radikulären Schmerzen vor, $1 \mathrm{RCT}$ im EERW-Design mit Hydromorphon mit 94 Patienten über 12 Wochen und eine Cross-over-Studie mit Morphin über 5 Wochen mit 55 Patienten. Die Ergebnisse bezüglich der Schmerzreduktion waren inkonsistent. Bezüglich der globalen Besserung, der Abbruchraten wegen Nebenwirkungen und der Häufigkeit schwe- 
Tab. 7 Wirksamkeit, Verträglichkeit und Sicherheit von Opioiden bei chronischen radikulären Schmerzen. (Modifiziert nach [145])

\begin{tabular}{|c|c|c|c|}
\hline Studiendauer (für RCT Dauer doppelblinde Phase) & 4-12 Wochen & 13-26 Wochen & $>26$ Wochen \\
\hline Anzahl RCTs und Patienten & $2 / 149$ & $\begin{array}{l}\text { Keine kontrollierte } \\
\text { Studie }\end{array}$ & $\begin{array}{l}\text { Keine kontrollierte } \\
\text { Studie } \\
\text { Keine Open-label- } \\
\text { extension-Studie }\end{array}$ \\
\hline Evidenzlevel & $\begin{array}{l}\text { Ib (keine quantitative Analyse möglich, da } \\
1 \text { RCT mit Parallel- und eine RCT mit EERW-De- } \\
\text { sign) }\end{array}$ & - & - \\
\hline Schmerzreduktion $50 \%$ und mehr & $\begin{array}{l}\text { Inkonsistent; Paralleldesign: kein statistisch sig- } \\
\text { nifikanter Unterschied zu Placebo. EERW-De- } \\
\text { sign: Statistisch signifikant Placebo überlegen }\end{array}$ & - & - \\
\hline Starke oder sehr starke globale Besserung & Keine Daten & - & - \\
\hline Reduktion Beeinträchtigungserleben & Beide Studien: kein Unterschied Placebo & - & - \\
\hline Abbruchraten wegen Nebenwirkungen & Beide Studien: kein Unterschied Placebo & - & - \\
\hline Häufigkeit schwerer Nebenwirkungen & $\begin{array}{l}\text { Beide Studien: kein statistisch signifikanter } \\
\text { Unterschied Placebo }\end{array}$ & - & - \\
\hline
\end{tabular}

Tab. 8 Wirksamkeit, Verträglichkeit und Sicherheit von Opioiden bei chronischen Schmerzen bei Polyneuropathie anderer Ätiologie als Diabetes. (Modifiziert nach [145])

\begin{tabular}{|c|c|c|c|}
\hline Studiendauer (für RCT Dauer doppelblinde Phase) & 4-12 Wochen & 13-26 Wochen & $>26$ Wochen \\
\hline Anzahl RCTs und Patienten & $2 / 111$ & Keine kontrollierte Studie & $\begin{array}{l}\text { Keine kontrollierte Studie } \\
\text { Keine Open-label-exten- } \\
\text { sion-Studie }\end{array}$ \\
\hline Evidenzlevel & la & - & - \\
\hline Schmerzreduktion $50 \%$ und mehr & $\begin{array}{l}\text { RD 0,26 }(0,09 ; 0,42) \\
\text { NNTB } 4(2,11)\end{array}$ & - & - \\
\hline Starke oder sehr starke globale Besserung & Keine Daten & - & - \\
\hline Reduktion Beeinträchtigungserleben & Keine Daten & - & - \\
\hline Abbruchraten wegen Nebenwirkungen & RD $0,05(-0,02 ; 0,13)$ & - & - \\
\hline Häufigkeit schwerer Nebenwirkungen & RD $0,02(-0,03 ; 0,07)$ & - & - \\
\hline
\end{tabular}

rer Nebenwirkungen fand sich kein Unterschied zu Placebo ([145]; - Tab. 7).

Es liegen zwei S2k-AWMF-LL zur Radikulopathie vor. Spezifische evidenzbasierte Empfehlungen wurden in den LL nicht formuliert. Für die zervikale Radikulopathie wird die Gabe von Opioiden in einem Stufenschema als Option erwähnt [44]. Für die lumbale Radikulopathie wird bei chronischen Schmerzen und Therapieresistenz eine „längere“ Anwendung unter Kontrolle der Wirksamkeit in seltenen Fällen als Option diskutiert [45].

\section{Modifiziert 2019: Polyneu- ropathie anderer Ätiologie als Diabetes*}

\subsection{Evidenzbasierte Empfehlung.}

4-12 Wochen: Opioidhaltige Analgetika sollten Patienten mit Polyneuropathie anderer Ätiologie als Diabetes als eine Therapieoption empfohlen werden. EL1a, Empfehlung ${ }^{* *}$. Starker Konsens (100\%;
$100 \%)$. ( ${ }^{*}$ ach Ansicht der Leitliniengruppe sollen Patienten mit alkoholischer Polyneuropathie und aktueller Substanzabhängigkeit nicht mit Opioiden behandelt werden. ${ }^{* *}$ Gründe der Abwertung des Empfehlungsgrades um 1 Stufe: Anzahl Patienten $<400$ in Metaanalyse)

\subsection{Konsensbasierte Empfehlung.}

13-26 Wochen: Opioidhaltige Analgetika können Patienten mit Polyneuropathie anderer Ätiologie als Diabetes als individueller Therapieversuch bei Therapieresponse als eine Therapieoption empfohlen werden. Offene Empfehlung. Starker Konsens $(100 \%$; $100 \%)$

\subsection{Konsensbasierte Empfehlung.}

>26 Wochen: Opioidhaltige Analgetika können Patienten mit Polyneuropathie anderer Ätiologie als Diabetes als individueller Therapieversuch bei Therapieresponse als eine Therapieoption empfohlen werden. Offene Empfehlung. Starker Konsens $(100 \% ; 100 \%)$

Kommentar: Es wurden 2 RCTs mit einem Cross-over-Design mit Tramadol und 111 Patienten mit einer Dauer von 4 Wochen gefunden. Die Ursachen der Polyneuropathie waren neben Diabetes mellitus Medikamente, Autoimmunerkrankungen und idiopathische Formen. Eine getrennte Analyse nach der Ursache der Polyneuropathie erfolgte nicht. Die Überlegenheit gegenüber Placebo in der Schmerzreduktion um 50\% und mehr war klinisch relevant. Es fanden sich keine Unterschiede zu Placebo in den Abbruchraten wegen schwerer Nebenwirkungen und in der Häufigkeit schwerer Nebenwirkungen ([145]; - Tab. 8).

Die S2k-Leitlinie zu neuropathischen Schmerzen stellt fest, dass Opioide wirksam sind, wobei aber die Nebenwirkungen und das Abhängigkeitspotenzial 
beachtet werden sollten. Hochpotente Opioide sowie das niederpotente Opioid Tramadol, das zusätzlich noch über eine noradrenerge und serotonerge Wiederaufnahmehemmung auf die endogene deszendierende Schmerzhemmung wirkt, können als Medikamente der dritten Wahl empfohlen werden [46].

\section{Modifiziert 2019: Evidenzba- sierte Empfehlung - rheumatoide Arthritis mit anhaltenden Schmerzen (ICD-10 M06.-) 9.1 Evidenzbasierte Empfehlung.}

4-12 Wochen: Opioidhaltige Analgetika können als ein individueller Therapieversuch angeboten werden. EL2b, offene Empfehlung*. Starker Konsens (100\%; $100 \%)$. ( ${ }^{*}$ Grund der Abwertung: nur eine RCT mit 20 Patienten ohne Intentionto-treat(ITT)-Analyse)

\subsection{Konsensbasierte Empfehlung.}

13-26 Wochen: Opioidhaltige Analgetika können als individueller Therapieversuch bei Respondern als eine Therapieoption angeboten werden. Offene Empfehlung. Starker Konsens (100\%; $100 \%)$

\subsection{Konsensbasierte Empfehlung.}

>26 Wochen: Opioidhaltige Analgetika können als individueller Therapieversuch bei Respondern als eine Therapieoption angeboten werden. Offene Empfehlung. Starker Konsens (100\%; $100 \%)$

Kommentar: Die aktualisierte Suche fand keine neue RCT mit Opioiden bei rheumatoider Arthritis. In einer RCT über 6 Wochen wurden 20 Patienten mit rheumatoider Arthritis über 6 Wochen mit Tilidin/Naloxon behandelt. Am Therapieende gaben 11 Patienten in der Verumgruppe eine geringere Schmerzintensität an als die 8 Patienten in der Placebogruppe $(p=0,05)$ (keine Intention-to-treat-Analyse). In der Tilidingruppe beendeten 5 Patienten vorzeitig die Therapie (2 wegen unzureichender Wirkung, 3 wegen Nebenwirkungen). In der Placebogruppe beendeten $2 \mathrm{~Pa}$ tienten vorzeitig die Therapie wegen unzureichender Wirkung. Da die Studie $<20$ Patienten pro Studienarm hatte [17], wurde sie nicht in die Metaanalysen für die Aktualisierung von LONTS aufgenommen.
Die S3-Leitlinie zum Management der frühen rheumatoiden Arthritis empfiehlt die Anwendung von NSAR zur symptomatischen Schmerztherapie. Die Anwendung von opioidhaltigen Analgetika zur symptomatischen Therapie von Patienten mit rheumatoider Arthritis sollte die Ausnahme darstellen, insbesondere bei Patienten im frühen Stadium der Erkrankung. Die Indikation sollte erst nach Ausschöpfung aller genannten Therapieoptionen gestellt werden. In Ausnahmefällen und bei bestehenden Kontraindikationen gegen NSAR kann ihre Gabe auch bei Patienten im frühen Stadium der Erkrankung gerechtfertigt sein [53].

\section{Restless-legs-Syndrom (ICD-10 G25)}

Neu 2019. 10.1 Evidenzbasierte Empfehlung: 4-12 Wochen: Opioide* sollten bei Patienten mit Restless-legs-Syndrom, die auf eine Therapie mit Levo-DOPA bzw. Dopaminagonisten nicht ausreichend ansprechen, als Therapieoption empfohlen werden. Evidenzgrad 1b, Empfehlung ${ }^{* *}$. Starker Konsens (100\%; 100\%)

Neu 2019. 10.2 Evidenzbasierte Empfehlung: 13-26 Wochen: Opioide* können bei Patienten mit Restless-legs-Syndrom bei Therapieresponse als individueller Therapieversuch als eine Therapieoption empfohlen werden. Evidenzgrad 3b. Offene Empfehlung. Starker Konsens (100\%; $100 \%)$

Neu 2019. 10.3 Konsensbasierte Empfehlung: >26 Wochen: Opioide* können als individueller Therapieversuch bei Patienten mit Restless-legs-Syndrom und Therapieresponse als eine Therapieoption empfohlen werden. Offene Empfehlung. Starker Konsens (100\%; $100 \%)$. ( Als einziges Opioid ist Oxycodon/Naloxon zur Second-line-Therapie des schweren bis sehr schweren idiopathischen RLS nach Versagen der dopaminergen Therapie zugelassen. ${ }^{* *}$ Gründe für Abwertung um 1 Stufe: $<400$ Patienten)

Kommentar: Die Bewegungsunruhe der Extremitäten beim Restlesslegs-Syndrom wird von einigen Patienten als schmerzhaft erlebt [89]. In einer 12-wöchigen placebokontrollierten RCT mit anschließender 40-wö- chiger Open-label-extension-Phase in 55 europäischen Studienzentren wurden 132 Patienten mit Oxycodon/Naloxon flexibler Dosis (Maximum $80 \mathrm{mg}$ Oxycodon; durchschnittliche Dosis in Studie 22 mg Oxycodon) und 144 Patienten mit Placebo behandelt. Ein Einschlusskriterium war das Versagen einer Therapie mit Levo-DOPA oder Dopaminagonisten. Ein sekundärer Endpunkt war die durchschnittliche Schmerzintensität am Therapieende. Diese lag bei Oxycodon/Naloxon bei $2,65(2,61)$ und bei Placebo bei $4,63(3,21)$ am Therapieende $(p<0,001)$. Die Abbruchrate wegen Nebenwirkungen in der randomisierten Phase war bei Oxycodon/Naloxon $15 \%$ und $7 \%$ bei Placebo $(p=0,02)$. Es fand sich ein signifikanter Unterschied in der Rate von schwerwiegenden therapiebedingten Nebenwirkungen (3\% vs. $0 \%)(p<0,02)$. In der Open-labelextension-Phase, in die 197 Patienten eingeschlossen wurden, wurden 3 schwere Nebenwirkungen unter Oxycodon/ Naloxon beobachtet. Entzugssymptome entwickelten sich bei einem Patienten nach Ende der Doppelblindphase und bei 2 Patienten nach Ende der Openlabel-extension-Phase [147].

In Deutschland ist die Zulassung für die Second-line-Therapie des schweren bis sehr schweren Restless-legsSyndroms nach Versagen der dopaminergen Therapie auf Oxycodon/Naloxon beschränkt.

\section{Parkinson-Syndrom (ICD-10 G20, G21)}

Neu 2019. 11.1 Evidenzbasierte Empfehlung: 4-12 Wochen: Opioide sollten nicht primär zur Schmerztherapie bei Patienten mit Parkinson-Syndrom angeboten werden. Evidenzgrad $1 b$, negative Empfehlung; ;*. Starker Konsens (100\%; 100\%). $\left({ }^{*}\right.$,Sollte nicht ${ }^{\prime \prime}=$ „kann einigen Personen angeboten werden“; ${ }^{\star \star}$ Gründe für Abwertung um 1 Stufe: $<400$ Patienten)

Neu 2019. 11.2 Konsensbasierte Empfehlung: Ein individueller Therapieversuch zur Schmerztherapie kann bei Patienten mit Parkinson-Syndrom angeboten werden. Starker Konsens (100\%; $100 \%$ ) 
Neu 2019. 11.2 Konsensbasierte Empfehlung: 13-26 Wochen: Opioidhaltige Analgetika können als individueller Therapieversuch zur Schmerztherapie bei Patienten mit Parkinson-Syndrom bei Therapierespondern als eine Therapieoption angeboten werden. Offene Empfehlung. Starker Konsens (100\%; $100 \%)$

11.3 Konsensbasierte Empfehlung: >26 Wochen: Opioidhaltige Analgetika können als individueller Therapieversuch zur Schmerztherapie bei Patienten mit Parkinson-Syndrom bei Therapieresponse als eine Therapieoption angeboten werden. Offene Empfehlung. Starker Konsens (100\%; $100 \%)$

Kommentar: Schmerzen verschiedener Lokalisation und Art werden von bis $\mathrm{zu} 90 \%$ der Patienten mit einem Parkinson-Syndrom berichtet, besonders häufig werden muskuloskeletale Schmerzen im Bereich der Wirbelsäule berichtet. Opioide werden im klinischen Alltag bei bis zu $15 \%$ der Patienten eingesetzt [158]. Mechanistisch wurde eine klinische Schmerzklassifikation vorgeschlagen, die neuropathische, nozizeptive und gemischte Ursachen unterscheidet und sowohl medikamentöse Optionen zur Optimierung des dopaminergen Status und zur Analgesie als auch nicht-medikamentöse Optionen berücksichtigt [152].

In einer RCT in 47 europäischen Studienzentren wurden 93 Patienten mit Oxycodon/Naloxon flexibel (Maximum $40 \mathrm{mg}$ Oxycodon/d; durchschnittliche Oxycodondosis $19 \mathrm{mg} / \mathrm{d}$ ) und $109 \mathrm{mit}$ Placebo über 16 Wochen behandelt. Bei $76 \%$ der Patienten lag ein muskuloskeletaler Schmerz vor, der jedoch nicht mechanistisch differenziert wurde. Die Schmerzintensität am Therapieende war $5,0(4,5,5,5)$ in der Oxycodongruppe versus 5,6 $(5,1,6,0)$ in der Placebogruppe $(p=0,058)$. Abbruchraten wegen Nebenwirkungen wurden nicht berichtet. Es fand sich kein statistisch signifikanter Unterschied in der Häufigkeit schwerer therapiebedingter Nebenwirkungen (5\% vs. $6 \%$ [148].

\section{Fibromyalgiesyndrom (ICD-10 M79.70)}

Modifiziert 2019. Kein Konsens zum Einsatz von Tramadol
Modifiziert 2019. Minderheitenvotum der Deutschen Gesellschaft für Rheumatologie

Evidenzbasierte Empfehlung: 4-12 Wochen: Tramadol sollte Patienten mit FMS als eine Therapieoption angeboten werden. EL1b, Empfehlung*. $\left({ }^{*}\right.$ Gründe der Abwertung des Empfehlungsgrades um 1 Stufe: Anzahl Patienten $<400$ in Metaanalyse)

Konsensbasierte Empfehlung: 13-26 Wochen: Tramadol kann Patienten mit FMS mit Therapieresponse als eine Therapieoption empfohlen werden. Offene Empfehlung

Konsensbasierte Empfehlung: >26 Wochen: Tramadol kann Patienten mit FMS und Therapieresponse als eine Therapieoption empfohlen werden. Offene Empfehlung

Modifiziert 2019. Minderheitenvotum der Deutschen Schmerzgesellschaft

Evidenzbasierte Empfehlung: 4-12 Wochen: Tramadol kann Patienten mit FMS als eine Therapieoption angeboten werden. EL1b, offene Empfehlung*. $\left({ }^{*}\right.$ Gründe der Abwertung des Empfehlungsgrades um 2 Stufen: Anzahl Patienten $<400$ in Metaanalyse; Evidenzlevel 1a für Wirksamkeit und geringere Risiken nichtmedikamentöser Verfahren [aerobes Training; kognitive Verhaltenstherapien])

Konsensbasierte Empfehlung: 13-26 Wochen: Tramadol kann Patienten mit FMS mit Therapieresponse als eine Therapieoption empfohlen werden. Offene Empfehlung.

Konsensbasierte Empfehlung: $>26$ Wochen: Tramadol kann Patienten mit FMS und Therapieresponse als eine Therapieoption empfohlen werden. Offene Empfehlung.

Kommentar: In der aktualisierten Literatursuche wurde keine neue RCT mit Opioiden beim FMS gefunden.

Es wurde eine placebokontrollierte RCT mit Tramadol mit einem Enriched-enrolment-randomized-withdrawal(EERW)-Design mit 69 Patienten über 6 Wochen durchgeführt [130]. Tramadol war Placebo in der Reduktion der Häufigkeit starker Schmerzreduktion (,a lot of pain relief“) statistisch signifikant überlegen. Tramadol führte zu statistisch signifikant höheren Abbruchraten wegen Nebenwirkungen als Placebo.

Die deutsche S3-Leitlinie zum FMS gibt eine negative Empfehlung zu starken Opioiden beim FMS. Die deutsche FMS-Leitlinie gibt keine evidenzbasierte Empfehlung zu Tramadol, da kein Konsens (nur mehrheitliche Zustimmung) in der Leitliniengruppe erzielt wurde [144].

In der Leitliniengruppe konnte kein Konsens bzw. keine mehrheitliche $\mathrm{Zu}$ stimmung für oder gegen Tramadol beim Fibromyalgiesyndrom erzielt werden. Folgende Argumente wurden gegen eine Empfehlung in der Leitlinienkonferenz vorgebracht:a) Die positive Wirkung von Tramadol ist möglicherweise durch die Hemmung der Aufnahme von Noradrenalin und Serotonin bedingt. Noradrenalin-Serotonin-Wiederaufnahmehemmer sind Placebo in der Schmerzreduktion beim FMS überlegen [154]. b) Das FMS ist häufig mit psychischen Störungen mit Leitsymptom Schmerz assoziiert. Die vorliegende Leitlinie spricht bei dieser klinischen Konstellation eine Kontraindikation für Opioide aus.

Begründung des Minderheitenvotums: Die Leitliniengruppe hat bei zwei anderen chronischen Schmerzsyndromen (Postzosterneuralgie, rheumatoide Arthritis) bei gleicher Datenlage (1 RCT mit Nachweis einer Wirksamkeit) die oben genannten Empfehlungen ausgesprochen. Es ist nicht konsistent, beim FMS andere Maßstäbe anzulegen. Einige Patienten mit FMS haben relevante nozizeptive [85] und evtl. auch neuropathische Schmerzkomponenten [78], welche eine Therapie mit Opioiden begründen können. Auch die European League Against Rheumatism (EULAR) gibt eine "Weak-for"-Empfehlung in ihrer Leitlinie zum Management des FMS [96]. Nach der klinischen Erfahrung einiger Teilnehmer der Leitliniengruppe als auch von Betroffenen der Deutschen Fibromyalgie Vereinigung profitieren einige Patienten mit FMS von einer Therapie mit Tramadol.

Redaktionelle Änderung: Konsensbasierte Empfehlung: Bei allen anderen nicht-tumorbedingten chronischen Schmerzen (siehe unten) kann eine Therapie mit opioidhaltigen An- 
algetika aufgrund unzureichender Datenlage nur als individueller Therapieversuch erwogen werden. Offene Empfehlung.

Mögliche Indikationen für eine kurz-, mittel- und langfristige Therapie mit opioidhaltigen Analgetika sind

13: Einige sekundäre Kopfschmerzen (z. B. bei Gefäßstörungen und nicht-vaskulären intrakraniellen Störungen, posttraumatisch) (ICD-10 G44.8).

Modifiziert 2019: starker Konsens (100\%; $100 \%)$

14: Chronische Schmerzen bei manifester Osteoporose. (Wirbelkörperfrakturen) (ICD-10 M80.-). Bestätigt 2019: starker Konsens

15: Chronische Schmerzen bei anderen entzündlich-rheumatischen Erkrankungen außer rheumatoider Arthritis (z.B. systemischer Lupus erythematodes, Spondylarthritiden) (ICD-10 M45-M49). Bestätigt 2019: starker Konsens

16: Chronische postoperative Schmerzen (z. B. Postthorakotomie-, Poststernotomie-, Postmastektomiesyndrom, nach Bauch- und Hernienoperationen, nach Gesichtsoperationen). Bestätigt 2019: starker Konsens

17: Chronischer Extremitätenschmerz bei ischämischen und entzündlichen arteriellen Verschlusskrankheiten (ICD-10 I70-I79). Bestätigt 2019: starker Konsens

18: Chronische Schmerzen bei Dekubitus Grad 3 und 4 (ICD-10 L89.2- und L89.3-). Bestätigt 2019: starker Konsens

19: Chronische Schmerzen bei fixierten Kontrakturen bei pflegebedürftigen Patienten. Modifiziert 2019: starker Konsens (100\%; $100 \%)$

20: Zentrale (zerebrale) neuropathische Schmerzen (z. B. nach Thalamusinfarkt, multiple Sklerose). Bestätigt 2019: starker Konsens

21: Chronisches CRPS Typ I und II. Bestätigt 2019: starker Konsens

22: Traumatische Trigeminusneuropathie (ICD-10 G50.9). Neu
2019: starker Konsens (100\%; $100 \%)$

23: Chronischer Unterbauchschmerz der Frau bei ausgeprägten Verwachsungen (ICD-10 N73.6) und/ oder multilokulärer Endometriose (ICD-10 N80.x). Neu 2019: Konsens (79\%; $76 \%$ )

Kommentar: Die oben genannten Indikationen sind weder durch kontrollierte Studien belegt noch gibt es eindeutige Hinweise für einen Schaden oder negative Effekte bzw. negative Aussagen aus anderen Leitlinien. Für einen individuellen Therapieversuch gelten die in Abschnitt III geschilderten Aussagen zur Praxis der Opioidtherapie in besonderer Weise.

\section{Indikation für interdisziplinäres Assessment}

Neu 2019. Konsensbasierte Empfehlung: Bei Unklarheiten zu Indikation einer LTOT kann ein interdisziplinäres Assessment mit anschließender Fallkonferenz erwogen werden [26]. Offene Empfehlung. Starker Konsens (96\%; $95 \%$ )

\section{Intrathekale Opioidtherapie}

Neu 2019. Konsensbasierte Feststellung: In seltenen Einzelfällen kann bei allen oben genannten potenziellen Indikationen im Falle einer Opioidsensitivität des Schmerzes und intolerablen Nebenwirkungen mehrerer oraler und transdermaler Opioide im Rahmen eines interdisziplinären Assessments eine intrathekale Opioidtherapie erwogen werden. Starker Konsens (100\%; 100\%)

Kommentar: Es liegen zur Wirksamkeit der intrathekalen Opioidtherapie bei Patienten mit Schmerzen nicht-maligner Ursachen keine RCTs vor, allerdings Kohortenstudien mit einem Follow-up von mehreren Jahren [65].

\section{Praxiswerkzeug}

- Intrathekale Opioidtherapie https://www.awmf.org/leitlinien/ detail/ll/145-003.html

\section{Kontraindikationen}

26. Primäre Kopfschmerzen (ICD10 G43.x, G44.0, G44.2, G44.8 [Kopfschmerz ohne begleitende strukturelle Läsion])

Bestätigt 2019. Evidenzbasierte Empfehlung: Alle primären Kopfschmerzen sollen nicht mit opioidhaltigen Analgetika behandelt werden. EL3b. Stark negative Empfehlung*. Starker Konsens. $\left({ }^{\star}\right.$ Gründe für Aufwertung der negativen Empfehlung: negatives Nutzen-SchadenVerhältnis von opioidhaltigen Analgetika; ethische Verpflichtungen.)

Kommentar: Die S1-Leitlinie zur Migräne stellt in einem Kommentar fest, dass Tramadol in der Kombination mit Paracetamol eine Wirksamkeit bei akuten Migräneattacken gezeigt hat. Opioidhaltige Analgetika und Tranquilizer sollten dennoch nicht zur Behandlung der Migräneattacke eingesetzt werden. Opioidhaltige Analgetika haben eine begrenzte Wirksamkeit, führen häufig zu Erbrechen, haben ein hohes Potenzial für eine Abhängigkeitsentwicklung in der bedarfsgesteuerten Anwendung und führen relativ rasch zu einem Kopfschmerz bei Medikamentenübergebrauch [47]. Eine Dauergabe retardierter Präparate, z.B. bei chronischer Migräne ist nicht untersucht und in der klinischen Erfahrung nicht wirksam, bei relevantem Schadenspotenzial.

Die S1-Leitlinie zum Spannungskopfschmerz stellt in einem Kommentar fest, dass retrospektive Analysen von Patienten mit chronischen täglichen Kopfschmerzen, die opioidhaltige Analgetika erhielten, zeigen, dass die Mehrzahl der Patienten entweder wegen Wirkungslosigkeit oder nicht $\mathrm{zu}$ tolerierender Nebenwirkungen der opioidhaltigen Analgetika oder wegen eines zunehmenden Medikamentengebrauchs die Therapie abbrach. Es gibt jedoch möglicherweise eine kleine Subgruppe, die von dieser Therapie anhaltend profitiert. Die Leitliniengruppe hält den Einsatz von opioidhaltigen Analgetika wegen des hohen Abhängigkeitspotenzials und der Hinweise auf eine vermehrte Chronifizierung von Kopfschmerzen unter opioidhaltigen Analgetika im Sinne eines 
medikamenteninduzierten Kopfschmerzes ausdrücklich für nicht indiziert [43].

\section{Schmerzen bei funktionellen/ somatoformen Störungen (ICD-10 F45.40)}

Bestätigt 2019. Konsensbasierte Empfehlung: Schmerzen bei funktionellen/somatoformen Störungen (ICD-10 F45.x) sollen nicht mit opioidhaltigen Analgetika behandelt werden. Stark negative Empfehlung*. Starker Konsens. (Gründe für Aufwertung der negativen Empfehlung: Negatives NutzenSchaden-Verhältnis von opioidhaltigen Analgetika; ethische Verpflichtungen.)

Kommentar: Die S3-Leitlinie zum Reizdarmsyndrom gibt eine negative Empfehlung zu opioidhaltigen Analgetika in der Therapie von Schmerzen beim Reizdarmsyndrom (RDS). Eine Empfehlung wird für Antidepressiva (trizyklische Antidepressiva, Serotoninwiederaufnahmehemmer [SSRI]), Spasmolytika und Ballaststoffe gegeben [94].

Die S3-Leitlinie zum FMS gibt eine stark negative Empfehlung für starke Opioide. An medikamentösen Therapieoptionen werden Amitriptylin und - bei komorbiden depressiven und Angststörungen - Duloxetin sowie bei komorbider generalisierter Angststörung Pregabalin empfohlen [144].

Die S2k-Leitlinie zum chronischen Unterbauchschmerz der Frau stellt in einem Kommentar fest, dass eine Behandlung mit opioidhaltigen Analgetika nicht erfolgen soll [51].

Die S3-Leitlinie zu nicht-spezifischen/ funktionellen/somatoformen Körperbeschwerden empfiehlt bei Patienten, die mit opioidhaltigen Analgetika behandelt sind, den Versuch einer Entwöhnungsbehandlung [128].
28. Chronischer Schmerz als (Leit-)Symptom psychischer Störungen (z. B. Depression [ICD-10 F43.2; F32; F33], anhaltende somatoforme Schmerzstörung [ICD-10 F45.40], generalisierte Angststörung [ICD-10 F41.1], posttraumatische Belastungsstörung [ICD-10 F43.1]) Modifiziert 2019. Konsensbasierte Empfehlung: Opioidhaltige Analgetika sollen bei psychischen Störungen mit Leitsymptom chronischer Schmerz nicht als Therapieoption angeboten werden. Stark negative Empfehlung*. Starker Konsens (100\%; $100 \%)$. ( ${ }^{\star}$ Gründe für Aufwertung der negativen Empfehlung: Negatives NutzenSchaden-Verhältnis von opioidhaltigen Analgetika; ethische Verpflichtungen.)

Kommentar: In der ambulanten (Haus- und Facharzt) und stationären Versorgung stellen manche Patienten mit psychischen Störungen bei der Schilderung ihrer Beschwerden chronische Schmerzen und nicht seelische Symptome in den Vordergrund. Die psychische Störung als Ursache der chronischen Schmerzen kann häufig nur durch eine vertiefte Exploration (z.B. durch Facharzt für Psychiatrie und Psychotherapie, Facharzt für psychosomatische Medizin, psychologischen Psychotherapeuten) und/oder den Verlauf (z.B. Remission der Schmerzen nach psychiatrisch-psychotherapeutischer Behandlung einer depressiven Störung) gestellt werden.

\section{Chronische Pankreatitis (ICD- 10 K86.-)}

Redaktionelle Änderung 2019. Evidenzbasierte Empfehlung: Opioidhaltige Analgetika sollten zur Schmerztherapie bei chronischer Pankreatitis nicht als Therapieoption für eine Langzeitanwendung angeboten werden. Negative Empfehlung. EL2b. Starker Konsens (100\%; 100\%)

Kommentar: Es wurden keine neuen kontrollierten Studien zu Opioiden zur Therapie chronischer Schmerzen bei chronischer Pankreatitis gefunden. Die Empfehlung bezieht sich auf die Therapie der chronischen Schmerzen. In der Akutschmerztherapie (akuter Schub einer chronischen Pankreatitis) kann eine kurzfristige Therapie mit Opioiden bei unzureichendem Ansprechen auf andere Analgetika erwogen werden.

Die deutsche Leitlinie zur chronischen Pankreatitis empfiehlt eine symptomatische Schmerztherapie nach dem WHOStufenschema [101] zur Überbrückung bis $\mathrm{zu}$ einer definitiven Therapie (z.B. endoskopisch oder chirurgisch).

In einer dänischen Studie war eine Opioidtherapie mit $>100 \mathrm{mg}$ Morphinäquivalent/d mit einer erhöhten Hospitalisierungsrate assoziiert [113].

Es gibt Hinweise, dass opioidhaltige Analgetika bei der chronischen Pankreatitis nicht wirksam sind. In einer offenen randomisierten Cross-overStudie wurden 18 Patienten mit chronischer Pankreatitis jeweils 4 Wochen mit transdermalem Fentanyl (durchschnittliche Studiendosis $36 \mu \mathrm{g} / \mathrm{h}$ ) oder retardiertem Morphin (durchschnittliche Studiendosis $128 \mathrm{mg} / \mathrm{d}$ ) behandelt. Alle Patienten waren mit opioidhaltigen Medikamenten vorbehandelt (keine Dosen genannt). Es erfolgte keine Washout-Phase. Beide Medikamente führten weder zu einer signifikanten Prä-postReduktion der Schmerzintensität noch zu einer Verbesserung der körperlichen Funktionsfähigkeit. Zwei Patienten brachen die Studie wegen Nebenwirkungen ab [109].

Primäre Maßnahme der Schmerztherapie ist im Falle von Alkohol- und/oder Tabakkonsum das Erreichen einer Abstinenz. Eine anhaltende Schmerzreduktion kann bei einem Teil der Patienten mit Obstruktion des Pankreasgangs durch operative und endoskopisch-interventionelle Verfahren erreicht werden [141].

In den USA ist in retrospektiven Kohortenstudien eine Zunahme der „opioid use disorder" bei Patienten mit chronischer Pankreatitis beschrieben [14].

\section{Chronisch-entzündliche Darmerkrankungen (ICD-10 K50.- und K51.-)}

Modifiziert 2019. Evidenzbasierte Empfehlung: Opioidhaltige Analgetika sollten zur Schmerztherapie bei chronisch-entzündlichen Darmerkrankungen nicht als Therapieoption für eine Langzeitanwendung angeboten werden. EL3b. Negative Empfehlung. Starker Konsens (100\%; $100 \%)$ 
Kommentar: Es wurden erneut keine kontrollierten Studien zu Opioiden zur Therapie chronischer Schmerzen bei chronisch-entzündlichen Darmerkrankungen gefunden. Die deutsche Leitlinie zum Morbus Crohn empfiehlt Metamizol, Paracetamol oder Opioide zur zeitlich befristeten (keine genaue Zeitangabe) symptomatischen Schmerztherapie im akuten Schub. Eine längerfristige Gabe (keine genaue Zeitangabe) von Opioiden zur symptomatischen Schmerztherapie wird diskutiert, aber wegen der damit verbundenen Risiken (negative Effekte auf Gastrointestinaltrakt und Immunsystem) nicht empfohlen [120].

In einer prospektiven Kohortenstudie mit US-amerikanischen Patienten gaben Patienten mit und ohne Opioide dieselbe Intensität der Bauchschmerzen an [35]. In einer retrospektiven Kohortenstudie einer britischen allgemeinärztlichen Datenbank war die Mortalität von Patienten mit Morbus Crohn bei Hochdosisgebrauch von starken Opioiden und von Patienten mit Colitis ulcerosa bei Gebrauch von starken Opioiden im mittleren und Hochdosisbereich im Vergleich zu Propensity-score-gematchten Patienten erhöht [20].

\section{Schädlicher Gebrauch opioidhaltiger Analgetika} Redaktionelle Änderung 2019. Konsensbasierte Empfehlung: Bei aktuellem schädlichem Gebrauch oder Weitergabe von Medikamenten an unberechtigte Personen und/oder schwerwiegendem Zweifel an verantwortungsvollem Gebrauch opioidhaltiger Analgetika (z.B. unkontrollierte Medikamenteneinnahmen und/ oder anhaltende fehlende Bereitschaft oder Unfähigkeit zur Einhaltung des Behandlungsplans) soll keine Therapie mit Opioiden begonnen werden. Starke Empfehlung. Starker Konsens

\section{Schwere affektive Störung (ICD-10 F32-34) und/oder}

\section{Suizidalität (ICD-10 R45.8)}

Bestätigt 2019. Konsensbasierte Empfehlung: Bei schwerer affektiver Störung und/ oder Suizidalität soll keine Therapie mit opioidhaltigen Analgetika begonnen wer- den. Stark negative Empfehlung. Starker Konsens

\section{Praxis der Opioidtherapie}

Vorbemerkung: Ein Qualitätskriterium von Leitlinien ist die Bereitstellung von Materialien, welche die einfache Anwendbarkeit der Leitlinienempfehlungen in der täglichen Arbeit unterstützen. Diese Materialien sollten einfach sein und - wenn möglich - ohne bzw. mit geringen Kosten zur Verfügung gestellt werden. Bei einigen der folgenden Empfehlungen werden daher sogenannte Praxiswerkzeuge für die behandelnden Ärzte aufgeführt. Es handelt sich dabei um Vorschläge der Steuer- und Konsensusgruppe.

Schlüsselempfehlungen der Leitlinie sind fett hervorgehoben.

\section{A. Maßnahmen vor Einleitung einer Therapie mit opioidhaltigen Analgetika}

\section{Neu 2019: Stellenwert von Opioiden bei nicht- tumorbedingten Schmerzen}

Konsensbasierte Empfehlung: Vor Einleitung einer Therapie mit Opioiden sollen die nicht-medikamentösen Therapieoptionen optimiert und medikamentöse Alternativen erwogen werden. Starke Empfehlung

Kommentar: In keiner deutschen Leitlinie $\mathrm{zu}$ einzelnen nicht-tumorbedingten Schmerzsyndromen sind Opioide die Therapie der 1. Wahl.

\section{Partizipative Entscheidungs- findung}

Bestätigt 2019. Konsensbasierte Empfehlung: Im Rahmen einer partizipativen Entscheidungsfindung sollen mit dem Patienten der mögliche Nutzen und Schaden einer Therapie mit opioidhaltigen Analgetika im Vergleich zu anderen medikamentösen Therapieoptionen sowie zu nicht-medikamentösen Behandlungsoptionen besprochen werden. Starker Konsens

Kommentar: Individuell relevante Risiken sollen im Aufklärungsgespräch angeführt werden, z. B. Sturzgefahr und
Verwirrtheit bei älteren Menschen oder Libidoverlust bei jüngeren Patienten.

\section{Wahl der Pharmakotherapie} Bestätigt 2019. Konsensbasierte Empfehlung: Die Wahl der Pharmakotherapie soll unter Berücksichtigung des vorliegenden chronischen Schmerzsyndroms, der Begleiterkrankungen des Patienten, von Kontraindikationen, Patientenpräferenzen, Nutzen und Schaden bisheriger Therapien und dem Nutzen-Risiko-Profil von medikamentösen und nicht-medikamentösen Therapiealternativen erfolgen. Starke Empfehlung. Starker Konsens

\section{Monotherapie mit opioid- haltigen Analgetika}

Redaktionelle Ergänzung 2019. Konsensbasierte Empfehlung: Eine alleinige Therapie mit opioidhaltigen Analgetika soll bei chronischen nichttumorbedingten Schmerzsyndromen nicht durchgeführt werden. Selbsthilfeangebote und physikalische und/ oder physiotherapeutische und/oder psychotherapeutische Verfahren (inkl. Patientenedukation) und/oder Lebensstilmodifikation sollen eine medikamentöse Schmerztherapie ergänzen. Starke Empfehlung. Starker Konsens

Kommentar: Die Auswahl der nichtmedikamentösen Behandlungen soll sich nach bestehenden deutschen Leitlinien für die jeweiligen Krankheiten mit Leitsymptom chronischer Schmerz richten.

\section{Anamnese und klinischer Status} Bestätigt 2019. Konsensbasierte Empfehlung: Eine allgemeine, sucht- und schmerzbezogene Anamnese sowie der körperliche und psychische Status des Patienten sollen erhoben und dokumentiert werden. Starke Empfehlung. Starker Konsens

Kommentar:

\section{Allgemeine Anamnese}

- Bisherige Krankengeschichte und Operationen, Begleiterkrankungen, Allergien, psychische Störungen inkl. Substanzmissbrauch oder -abhängigkeit

- Ausführliche Medikamentenanamnese (inkl. Wechsel- und Nebenwirkungen, Einnahmegewohnheiten) 
- Sorgfältige körperliche Untersuchung einschließlich einer funktionellen Untersuchung des Bewegungsapparats; neurologische und ggf. neurophysiologische Untersuchung

- Überprüfung des aktuellen Funktionsniveaus

- Ggf. diagnostische Nervenblockaden

- Ggf. Laboruntersuchungen zu Leberund Nierenfunktion

- Ggf. Urinuntersuchung und weitere Laboruntersuchungen zur Überprüfung der Einnahme von nicht angegebenen illegalen Substanzen, Alkohol und Medikamenten.

- Ggf. spezielle Diagnostik bezüglich Erkrankungen und Zuständen, die Anwendungsbeschränkungen für opioidhaltige Analgetika darstellen können

- Feststellung, dass eine CNTSSchmerzart vorliegt, bei der eine Anwendung opioidhaltiger Analgetika erwogen werden kann

\section{Schmerzanamnese}

- Schmerzintensität auf einer numerischen oder visuellen Analogskala mit Angaben zu "momentaner Schmerz", „geringster sowie stärkster Schmerz während der letzten Woche" und „durchschnittlicher Schmerz während der letzten Woche(n)“

- Schmerzart und mögliche Grundkrankheiten

- Schmerzort, -dauer, -variabilität und -charakter

- Ursachenvermutungen des Patienten

- Einstellung auf die bisherige Schmerzmedikation (Schmerzintensität nach Anwendung, Dauer der Schmerzlinderung, alle Nebenwirkungen)

- Manifestationen des Schmerzes im Verhalten und Ausdruck des Patienten

- Beurteilung des sozialen Umfelds, Familie, soz. Verstärker, Rentenantrag, Arbeitssituation etc.

- Beanspruchung/Belastung durch den Schmerz

- Beurteilung schmerzbedingter Funktionseinschränkungen

- Aktuelle berufliche Tätigkeiten und Gefährdungen am Arbeitsplatz
- Aktuelle und frühere medikamentöse und andere Schmerzbehandlungen

- Erwartungen an die weitere Schmerztherapie

\section{Beurteilung des Funktionsniveaus}

- Kognitive Funktionsfähigkeit (Aufmerksamkeit, Konzentration, Gedächtnis)

- Berufstätigkeit bzw. Arbeitsfähigkeit

- Lebensfreude, soziale Kontaktpflege

- Schlaf

- Mobilität

- Sexuelle Funktionsfähigkeit

- Selbstachtsames bzw. -versorgendes Verhalten

- Hausarbeit, Hobbys, Aktivitäten

\section{Praxiswerkzeuge}

- Deutsche Version des Kurzschmerzfragebogens Brief Pain Inventory (BPI)

https://www.dnqp.de/fileadmin/ HSOS/Homepages/DNQP/

Dateien/Expertenstandards/

Schmerzmanagement_in_der_ Pflege_bei_akuten_Schmerzen/ Schmerz_BPI.pdf

- Deutscher Schmerzfragebogen DSF https://www.schmerzgesellschaft.de/ schmerzfragebogen

- Assessmentempfehlungen für Senioren im deutschsprachigen Raum https://www.awmf.org/leitlinien/ detail/ll/145-003.html

\section{Psychosoziale Anamnese}

Modifiziert 2019. Konsensbasierte Empfehlung: Eine psychosoziale Anamnese und ein Screening auf aktuelle und/oder frühere psychische Störungen sollen von der behandelnden Ärztin/dem behandelnden Arzt durchgeführt werden. Starke Empfehlung. Starker Konsens (100\%; $100 \%)$

Kommentar: Die psychosoziale Anamnese soll aktuelle psychosoziale Belastungen (z. B. Arbeit, Familie) erfassen. Ein Screening auf psychische Störungen soll vermehrte Depressivität, Angst, körperliche Symptombelastung sowie Substanzmissbrauch erfassen.

Eine multimodale schmerztherapeutische Behandlung erfordert eine interdisziplinäre Diagnostik durch mindes- tens zwei Fachdisziplinen. Eine Fachdisziplin ist obligatorisch eine psychiatrische oder psychosomatische oder psychologisch-psychotherapeutische Disziplin; siehe OPS 8.91x [26, 56].

\section{Praxiswerkzeuge}

- Screening-Instrument für Angst und Depression PHQ 4

http://de.wikipedia.org/wiki/Datei: PHQ-4.TIF

\section{Screening auf Alkoholabhängig- keit}

a. Audit-C-Fragebogen

http://www.bundesaerztekammer.de/ downloads/AlkAUDITCFragebogen. pdf

b. CAGE-Test http://www.zuefam.ch/pdf/cagetest. pdf

\section{Geriatrische Depressionsskala}

- https://www.geriatrie-bochum.de/ assessment/geriatrische-depressionsskala-gds.php

\section{Fachpsychotherapeutische Konsiliaruntersuchung}

Bestätigt 2019. Konsensbasierte Empfehlung: Bei Hinweisen auf eine psychische Störung sollte eine Vorstellung bei einem Facharzt für Psychiatrie und Psychotherapie oder Psychosomatische Medizin und Psychotherapie oder einem ärztlichen bzw. psychologischen Psychotherapeuten erfolgen. Empfehlung. Starker Konsens

Kommentar: Eine Diagnostik auf psychische Störungen kann auch von Hausärzten oder Ärzten mit der Zusatzbezeichnung „Spezielle Schmerztherapie“ mit Kompetenz in psychosomatischer Grundversorgung durchgeführt werden.

\section{Therapieziele}

Bestätigt 2019. Konsensbasierte Empfehlung: Mit dem Patienten sollen individuelle und realistische Therapieziele erarbeitet werden. Starke Empfehlung. Starker Konsens

Kommentar: Patienten mit chronischen Schmerzen haben häufig hohe Erwartungen an eine medikamentöse Schmerzreduktion. In einer Befragung von Patienten einer US-Schmerzklinik gaben 52 Patienten mit chroni- 
schen Rückenschmerzen eine 58\%ige Schmerzreduktion (auf 2,2 auf 11-stufiger Skala) und $68 \%$ ige Reduktion des Beeinträchtigungserlebens (auf 1,8 auf 11-stufiger Skala) als Kriterium für eine erfolgreiche Therapie an [110].

Aus medizinischer Sicht sinnvolle Ziele einer Therapie (= Therapieresponse) sind eine individuell angemessene Verbesserung der Funktionsfähigkeit im Alltag (z. B. Arbeitswiederaufnahme, „wieder Rasen mähen können“; „sich selbst versorgen können") und/oder eine mindestens $30 \%$ ige Schmerzreduktion [62].

\section{Aufklärung}

Bestätigt 2019. Konsensbasierte Empfehlung: Eine dokumentierte mündliche und/oder schriftliche Aufklärung inkl. verkehrs- und arbeitsplatzrelevanter Aspekte des Patienten (evtl. auch der Familie und/oder Betreuer) soll erfolgen. Starke Empfehlung. Starker Konsens

Kommentar: Inhalte der Aufklärung

\section{Allgemeine Aspekte}

- Ziele und Erwartungen an die Therapie

- Behandlungsalternativen und ergänzende Maßnahmen

- Erfolgsabhängigkeit der Therapie vom individuellen Fall

- Notwendigkeit regelmäßiger Neubewertung der Behandlung

- Notwendigkeit, die Verordnung des opioidhaltigen Medikaments einem einzigen Arzt zu überlassen, sowie die genauen Regelungen für eine ggf. erforderliche Vertretung

- Instruktionen bzgl. der genauen Einnahme und Dosierung des Medikaments

- Hinweise auf Wechselwirkungen mit anderen Medikamenten

- Prophylaktische Behandlung unerwünschter Arzneimittelwirkungen, z. B. Obstipation

- Risiken des gleichzeitigen Konsums von Alkohol oder sedierender Medikamente

- Verantwortlichkeiten des Patienten: Einhaltung des Behandlungsplans, regelmäßige Rückmeldung an den behandelnden Arzt; z. B. durch ein Schmerztagebuch
- Sichere Aufbewahrung der opioidhaltigen Medikamente

- Hinweis auf die sichere Entsorgung der vom Patienten nicht eingenommenen Opioide entsprechend den einschlägigen betäubungsmittelrechtlichen Vorschriften

- Verbot der Weitergabe opioidhaltiger Medikamente

- Konsequenzen bei Non-Adhärenz

- Mitnahme opioidhaltiger Medikamente ins Ausland

\section{Risiken und Nebenwirkungen}

- Information über unerwünschte Arzneimittelwirkungen, die sowohl kurzfristig als auch erst bei längerer Anwendung von Opioiden auftreten können: u. a. Risiken physischer Abhängigkeit und der Entwicklung einer Abhängigkeitserkrankung, Obstipation, Übelkeit, Sedierung, Pruritus, Schwindel, Erbrechen, verringerte kognitive Leistungsfähigkeit, Müdigkeit, Mundtrockenheit, verstärktes Schwitzen, Kopfschmerz, Einschränkung der emotionalen Schwingungsfähigkeit, Verlust des sexuellen Verlangens, Auswirkungen auf die Hypophysen-GonadenAchse; atmungsbezogene Störungen

- Mögliche negative Auswirkungen auf Fahrsicherheit sowie Tätigkeiten am Arbeitsplatz (z. B. Arbeit an Maschinen, Steuertätigkeit) und Freizeit (z. B. Hausarbeit, Gartenarbeit, Sport)

\section{Praxiswerkzeug}

- Informationsblatt zur Opioidtherapie https://www.awmf.org/leitlinien/ detail/1l/145-003.html

\section{Aufklärung über Risiken einer Opioidtherapie}

Neu 2019. Evidenzbasierte Empfehlung: Patienten sollen auf folgende mögliche Risiken einer Langzeitanwendung von Opioiden hingewiesen werden: Erhöhtes Sturzrisiko; sexuelle Funktionsstörungen; endokrine Funktionsstörungen; Atemstörungen. EL3b. Starker Konsens (94\%, 94\%)

Kommentar: Eine Cochrane-Übersicht von systematischen Übersichten von randomisierten klinischen Studien mit Opioiden bei CNTS fand in den Studien keine Angaben zur Häufigkeit folgender Nebenwirkungen: Sucht, kognitive Dysfunktion, depressive oder andere emotionale Störungen, Hypogonadismus und andere endokrine Dysfunktionen, Atemdepression, sexuelle Funktionsstörungen, Schlafapnoe und andere schlafbezogene Atemstörungen [64]. Kohortenstudien zur Häufigkeit der genannten potenziellen Nebenwirkungen unterliegen - wie RCTs auch methodischen Limitationen [122].

Eine systematische Übersichtsarbeit mit Metaanalyse fand eine Assoziation von Sturzrisiko und Opioiden mit einer Odds Ratio von 1,60 (1,35-1,91). In zwei der analysierten Studien fanden sich Hinweise, dass das Risiko insbesondere in der Einstellungsphase erhöht ist [140, 153].

Die Datenlage zur Immunsuppressivität von Opioiden ist komplex und deren Relevanz für die klinische Anwendung bei chronischen Schmerzen sehr limitiert. Die Übertragbarkeit akuter Effekte auf die chronische Anwendung von Opioiden ist nicht gesichert. Eine retrospektive Kohortenstudie zeigte, dass möglicherweise ein erhöhtes Risiko schwerer Infektionen unter einer Langzeittherapie mit von den Autoren als immunsuppressiv eingeschätzten Opioiden (Morphin, Fentanyl, Methadon) im Vergleich zu nicht-immunsuppressiven Opioiden (Oxycodon, Oxymorphon, Tramadol) besteht [157]. Bei Patienten mit rheumatoider Arthritis fand sich eine Assoziation von schweren Infektionen bei Neubeginn einer Therapie mit immunsuppressiven Opioiden (Morphin, Fentanyl, Methadon): IRR (,incidence rate ratio“) 2,01 (1,52, 2,66), IRR $1,72(1,33,2,23)$, IRR 2,38 (1,65, 3,42 ) [156]. Buprenorphin wurde in diesen Studien nicht untersucht, wird aber als nicht-immunsuppressiv eingeordnet [71, 117]. Zu Tapentadol liegt bisher nur eine tierexperimentelle Studie vor [70]. Eine systematische Übersichtsarbeit (1 RCT, 5 Querschnittsstudien) kam zur Schlussfolgerung, dass eine schwache Evidenz besteht, dass Opioide bei einer Langzeitanwendung ( $\geq 4$ Wochen) bei Patienten mit CNTS zu Veränderungen der „natural killer cells“ führen [57].

Opioide können bei Männern und Frauen zu einem Hypogonadismus füh- 
ren $[3,36]$. Morphin sollte laut Fachinformation Männern und Frauen im zeugungs- und gebärfähigen Alter nur dann verabreicht werden, wenn eine wirksame Verhütung sichergestellt ist [123].

Eine systematische Übersichtsarbeit mit Metaanalyse fand ein erhöhtes Risiko für ein Delir unter Opioidtherapie, OR 2,5 (1,2-5,2) [34].

Die Datenlage zu einer erhöhten Mortalität einer Opioidtherapie bei CNTS im Vergleich zu keiner analgetischen Therapie bzw. Therapie mit nicht-opioidhaltigen Analgetika ist aufgrund möglicher methodischer Verzerrungen („confounding by indication" = Patienten mit stärkeren Schmerzen bzw. mehreren Begleiterkrankungen erhalten eher Opioide als Patienten mit geringeren Schmerzen bzw. weniger Begleiterkrankungen) [159] und selektierter Patientenpopulationen [126] nicht eindeutig.

\section{Fahrsicherheit mit Opioiden}

Bestätigt 2019. Konsensbasierte Empfehlung: Eine Aufklärung über verkehrs- und arbeitsplatzrelevante Aspekte der Therapie soll der Arzt vor Beginn einer Behandlung mit opioidhaltigen Analgetika durchführen und dokumentieren. Starke Empfehlung. Starker Konsens

\section{Praxiswerkzeug}

- Infoblatt Fahrsicherheit unter

Opioiden

https://www.awmf.org/leitlinien/

detail/1l/145-003.html

\section{Titration und Fahrsicherheit} Bestätigt 2019. Konsensbasierte Empfehlung: Patienten sollen darauf hingewiesen werden, dass sie während der Dosisfindungsphase und bei Dosisänderungen nicht Auto fahren sollen. Starke Empfehlung. Starker Konsens

\section{Praxiswerkzeug}

- Infoblatt Fahrsicherheit unter

Opioiden

https://www.awmf.org/leitlinien/

detail/ll/145-003.html

\section{Titration bzw. Dosisänderung und Arbeitsplatz}

Bestätigt 2019. Konsensbasierte Empfehlung: Eine mögliche Gefährdung am
Arbeitsplatz sollte berücksichtigt werden. Empfehlung. Starker Konsens

Kommentar: Wenn berufliche Tätigkeiten mit unklarem oder erhöhtem Gefährdungspotenzial verbunden sind, kann erwogen werden, den betriebsbetreuenden Arbeitsmediziner (Betriebsarzt) zur Beratung und Unterstützung des Mitarbeiters hinzuzuziehen.

\section{Kombinationstherapie von Opioiden mit Tranquilizern}

Neu 2019. Evidenzbasierte Empfehlung: Eine Kombinationstherapie von Opioiden mit Tranquilizern sollte nicht durchgeführt werden. EL2b. Negative Empfehlung. Starker Konsens (100\%; $100 \%)$

Kommentar: In einer retrospektiven Querschnittsstudie im Jahr 2014 mit 4.028.618 Versicherten von 69 deutschen gesetzlichen Krankenversicherungen mit einer LTOT wegen chronischer nichttumorbedingter Schmerzen (CNTS) erhielten ca. $12 \%$ Verordnungen von Tranquilizern [84].

Notfallaufnahmen wegen Überdosierungen: In einer US-amerikanischen Studie mit 315.428 Privatversicherten im Alter von 18 bis 64 Jahren (Ausschluss früherer oder aktueller Krebserkrankungen) in den Jahren 2001-2013 war die kombinierte Einnahme von Opioiden (mindestens 1 Dosis) mit Tranquilizern im Vergleich zu einer Monolangzeittherapie mit Opioiden mit einem erhöhten Risiko für eine Vorstellung in der Notaufnahme oder einer stationären Aufnahme wegen einer Überdosierung (adjustierte OR $1,81[1,67,1,96]$ ) (keine absoluten Werte genannt) assoziiert [146].

Missbräuchliche/süchtige Verwendung: In einer retrospektiven Querschnittsstudie im Jahr 2014 mit 4.028.618 Versicherten von 69 deutschen gesetzlichen Krankenversicherungen mit einer LTOT wegen CNTS waren Krankenhausaufenthalte mit Diagnosen von psychischen und Verhaltensstörungen aufgrund von Alkohol, Opioiden, Tranquilizern und multiplem Substanzgebrauch sowie Vergiftungen durch Betäubungsmittel mit der Verschreibung von Tranquilizern (Odds Ratio [OR] 3,63 [3,03; 4,36]) assoziiert [84]. In einer retrospektiven Querschnittsstudie im Jahr 2012 mit
800.000 Versicherten der BEK waren Krankenhausaufenthalte mit Diagnosen von psychischen und Verhaltensstörungen wegen Alkohol, Opioiden, Tranquilizern und multiplem Substanzgebrauch und Vergiftungen durch Betäubungsmittel mit der Verschreibung von Tranquilizern (Odds Ratio [OR] 3,44 [1,80; 6,56]) assoziiert [100].

Todesfälle: In einer US-amerikanischen Studie mit ehemaligen Armeeangehörigen in den Jahren 2004-2009 wurden 2400 Todesfälle im Zusammenhang mit einer Opioidmedikation und Tranquilizern beschrieben, Hazard Ratio (HR) von 3,86 (3,49; 4,26) (keine Angabe absoluter Zahlen) [115].

In einer prospektiven Beobachtungsstudie mit 1-jähriger Nachbeobachtung mit US-amerikanischen Seniorenheimbewohnern wurden 478 Todesfälle in Zusammenhang mit einer Überdosierung $(0,022 \%$ pro Jahr bei 2.182 .374 Personen) festgestellt. Todesraten aufgrund von Überdosierungen waren unter einer Kombinationstherapie von Opioiden und Benzodiazepinen 10-mal höher (7,0 pro 10.000 Personenjahre $[6,3,7,8])$ als unter Opioiden allein (0,7 pro 10.000 Personenjahre $[0,6,0,9]$ ) (keine absoluten Zahlen genannt) [39].

In einer Studie an US-Veteranen wurde der Zusammenhang einer leitliniengerechten Langzeitopioidtherapie und der 1-Jahres-Mortalität untersucht. Diese war bei einer Komedikation mit Benzodiazepinen (HR 1,39 [1,12, 1,66]) erhöht. Eine Abnahme der Mortalität fand sich bei begleitender Psychotherapie (HR 0,62 [0,51, 0,71]) und körperlicher Rehabilitation (HR 0,81 95\%-CI $0,67,0,98)$. Wurde im Falle einer Substanzabhängigkeit eine Suchttherapie durchgeführt, fand sich ebenfalls eine Abnahme der Mortalität (HR 0,47 [0,32, 0,68]) [75].

Eine retrospektive Kohortenstudie aus den USA (Medicaid-Patienten) zeigte einen Anstieg von Todesfällen in Abhängigkeit von der Opioiddosis bei mindestens einer Opioidverschreibung: Im Vergleich zu Patienten mit einer Morphinäquivalentdosis (MEQ) von 1 bis $19 \mathrm{mg}$ und keiner Einnahme von Sedativa war das Risiko bei einer Kombination mit Tranquilizern 
wie folgt erhöht: 1-19 mg/d: „adjusted“ (aHR 5,6 [1,6-19,3]); 20-49 mg/d (aHR, 6,2 [1,9-20,0]), 50-89 mg/d (aHR, 11,9 $[3,7-38,8]), \quad 90-119 \mathrm{mg} / \mathrm{d} \quad(\mathrm{aHR}, \quad 15,8$ $[4,6-53,9])$ und $>120 \mathrm{mg} / \mathrm{d}$ (aHR, 20,3 [6,4-64,3]; [76]).

Die Aussagekraft der Studien ist durch den retrospektiven Charakter der meisten Studien und mögliche konfundierende Variablen (z. B. „confounding by indication“) eingeschränkt.

\section{B. Durchführung einer Therapie mit opioidhaltigen Substanzen}

\section{Differenzialindikation einzelner opioidhaltiger Analgetika}

Bestätigt 2019. Evidenzbasierte Empfehlung: Eine Empfehlung hinsichtlich der Bevorzugung eines einzelnen opioidhaltigen Analgetikums kann nicht gegeben werden. EL1a, starker Konsens

Neuer Kommentar: In den aktualisierten systematischen Übersichtsarbeiten von placebokontrollierten RCTs mit einer mindestens 4-wöchigen doppelblinden Phase fanden sich bei neuropathischen Schmerzen [145] und Rückenschmerzen [118] keine statistisch signifikanten globalen Subgruppenunterschiede bezüglich Schmerzreduktion von $50 \%$ und mehr, Beeinträchtigungserleben und Verträglichkeit zwischen den einzelnen Opioiden. In der systematischen Übersicht beim Arthroseschmerz [155] fand sich ein statistisch signifikanter globaler Subgruppenunterschied bezüglich Schmerzreduktion von $50 \%$ und mehr und Verträglichkeit, aber nicht für Beeinträchtigungserleben zwischen den einzelnen Opioiden.

Nach der klinischen Erfahrung der Steuergruppe kann die individuelle Wirksamkeit bzw. Verträglichkeit von Opioiden unterschiedlich sein. Diese klinische Erfahrung gilt auch für Tilidin/ Naloxon, dem in Deutschland am häufigsten verordneten Opioid [15], welches wegen fehlender RCTs nicht in die eigenen systematischen Übersichtsarbeiten bzw. die anderer Autoren einbezogen wurde. Daher gelten die Empfehlungen auch für Tilidin/Naloxon. Mögliche Gründe für das unterschiedliche Ansprechen auf Opioide sind genetische, physiologische sowie pharmakinetische und -dynamische Faktoren.

\section{Differenzialindikation der Applikationsform von opioidhaltigen Analgetika \\ Bestätigt 2019. Evidenzbasierte Empfeh-} lung: Eine Empfehlung hinsichtlich der Bevorzugung des oralen bzw. transdermalen Applikationswegs opioidhaltiger Analgetika kann nicht gegeben werden. EL1a, starker Konsens

Kommentar: Bei der systematischen Literatursuche wurden keine neuen randomisierten Direktvergleiche von oralen mit transdermalen Systemen gefunden. Nach der klinischen Erfahrung der Leitliniengruppe sind transdermale Systeme bei starken Schwankungen der Schmerzintensität während des Tages und in der Nacht nicht geeignet.

\section{Differenzialindikation von opioidhaltigen Analgetika}

Bestätigt 2019. Konsensbasierte Empfehlung: Bei der Auswahl eines opioidhaltigen Analgetikums und seiner Applikation sollen Begleiterkrankungen des Patienten, Kontraindikationen für transdermale Systeme oder eine orale Einnahme, das Nebenwirkungsprofil des opioidhaltigen Analgetikums sowie Patientenpräferenzen berücksichtigt werden. Starke Empfehlung. Starker Konsens

Kommentar: Trotz der umfangreichen Datenlage zur Therapie von chronischen Rücken- und Arthroseschmerzen ist das individuelle Ansprechen eines Patienten auf eine medikamentöse Therapie nicht vorhersagbar. Nach der klinischen Erfahrung der Leitliniengruppe können erhebliche inter- und intraindividuelle Unterschiede in der Wirksamkeit und Verträglichkeit verschiedener opioidhaltiger Analgetika bestehen.

\section{Praxiswerkzeuge}

- (Siehe https://www.awmf.org/ leitlinien/detail/ll/145-003.html)

- Opioidhaltige Analgetika bei Leberinsuffizienz

- Opioidhaltige Analgetika bei Niereninsuffizienz

- Hinweise der AkdÄ zur Anwendung von Fentanylpflastern

\section{Arzneimittelinteraktionen}

Neu 2019. Konsensusbasierte Empfehlung: Bei der Verordnung von opioidhaltigen Arzneimitteln soll auf mögliche Abschwächung bzw. Verstärkung der Opioidwirkung durch andere Medikamente und auf ein mögliches Serotoninsyndrom und anticholinerges Syndrom geachtet werden. Starker Konsens (100\%; $100 \%)$

\section{Kommentar:}

\section{Atemdepression}

Die kanadische Gesundheitsbehörde Health Canada hat die kanadischen Bürger in einer Mitteilung vom 17.09.2019 auf das erhöhte Risiko einer Überdosis von Opioiden und anderer schwerer Nebenwirkungen bei der gleichzeitigen Einnahme von Pregabalin bzw. Gabapentin hingewiesen [86]. In einer kanadischen bevölkerungsbasierten „nested case-control study“ (Fall-KontrollStudie, bei der die Fälle und Kontrollen aus der Population einer Kohortenstudie gezogen werden), welche Daten von 1997 bis 2013 berücksichtigte, war die Mortalität von Patienten bei einer Kombinationstherapie von Opioiden und Gabapentin im Vergleich zu einer Monotherapie mit Opioiden erhöht: OR 1,99 (1,61-2,47) [77].

Die US Food and Drug Administration hat in einer Mitteilung vom 19.12.2019 auf das Risiko einer Atemdepression und einer tödlichen Opioidüberdosierung bei einer Kombinationstherapie von Opioiden mit Pregabalin und Gabapentin hingewiesen [67].

Veränderungen der Bioverfügbarkeit

Der Metabolismus einzelner Opioide kann durch die Komedikation moduliert sein und zu Veränderungen der Bioverfügbarkeit führen. Durch Hemmung der metabolisierenden Enzyme steigen die Opioidplasmaspiegel und es drohen vermehrt Nebenwirkungen. Umgekehrt kann durch Enzyminduktion die Opioidkonzentration sinken und damit das Therapieansprechen ausbleiben. Tramadol ist ein Prodrug. Erst durch Biotransformation wird der opioidanalgetische Metabolit gebildet. Bei gleichzeitiger Anwendung von Enzyminhibitoren mit Tramadol wird dessen Funktionalisierung verhindert und ein analgetischer Effekt kann gemindert sein [116]. 
Serotoninsyndrom

Kombination von neurologischen Symptomen (Rigor, Tremor, Akathisie, Myoklonien, Krampfanfälle, Parästhesien, generalisierte Reflexsteigerung, Verwirrtheit, Somnolenz bis hin zum Koma, Desorientiertheit, Erregungszustände und agitiertes Delir) und vegetativen Symptomen (Schwitzen, Diarrhö, Übelkeit, Erbrechen, Bauchschmerzen, Tachykardie) bedingt durch erhöhten Serotoninspiegel [68]. Die Symptome bilden sich häufig innerhalb von Stunden aus. Mehr als 75\% der Patienten sind nach einem Tag symptomatisch. Bei älteren Patienten kann es jedoch zu einer Verzögerung kommen, erklärbar durch eine reduzierte Synthese von Serotonin und eine verringerte Rezeptordichte. Die drei Symptomgruppen treten in verschiedenen Schweregraden auf.

Das Serotoninsyndrom ist selten, aber gefährlich und kann zum Tod führen. Häufig werden Symptome in einem subklinischen oder leichtgradigen Stadium möglicherweise übersehen und nicht diagnostiziert. Das Serotoninsyndrom ist im Grunde eine Serotoninintoxikation. Es liegt pathophysiologisch ein Überangebot des Neurotransmitters Serotonin vor. Bewusstseinsstörungen, neuromuskuläre Hyperaktivität und autonome Instabilität bilden die Trias neuroexzitatorischer Symptome. Das Serotoninsyndrom kann zwar häufig auch bei hohen Dosen unter Monotherapie auftreten, die meisten Intoxikationen finden jedoch bei Kombination von zwei oder mehr serotonerg wirkenden Pharmaka statt. Oxycodon, Fentanyl, Tapentadol und Tramadol sind Opioide mit einem Risiko für ein Serotoninsyndrom, für die es Fallberichte gibt [133].

\section{Anticholinerges Syndrom}

Ein anticholinerges Syndrom kann durch einzelne oder eine Kombination von anticholinerg wirkenden Medikamenten (sogenannte anticholinerge Last) hervorgerufen werden [90]. Besonders ältere Patienten sind empfindlich für anticholinerge Nebenwirkungen.

Peripheres anticholinerges Syndrom: Obstipation, Harnverhalt, Tachykardie, Hypertonie, Mydriasis, trockene Haut und Schleimhäute
Zentrales anticholinerges Syndrom: Minderung der Vigilanz, Aggressivität, Agitiertheit, Halluzinationen, Koma, Schwindel und Dysarthrie.

Bei in der Schmerzmedizin eingesetzten Substanzen ist vor allem auf eine hohe anticholinerge Last von Opioiden in Kombination mit Antidepressiva und Neuroleptika zu achten.

Die Aussagekraft der Empfehlung ist durch das Fehlen ausreichender Daten zur Prävalenz der klinischen Relevanz der genannten Interaktionen und der Gefahr von „hyperalertness“ eingeschränkt.

\section{Praxiswerkzeug}

- Arzneimittelinteraktionen https://www.awmf.org/leitlinien/ detail/ll/145-003.html

\section{Konsensbasierte Emp- fehlung: bundeseinheitlicher Medikationsplan}

Neu 2019. Für Patienten mit einer Langzeittherapie mit opioidhaltigen Analgetika soll die Medikation mit Opioiden im bundeseinheitlichen Medikationsplan aufgenommen werden. Starker Konsens (100\%; $100 \%)$

Kommentar: Nach $₫ 31$ a SGB V haben Patienten einen Anspruch auf einen Medikationsplan durch die behandelnden Ärzte. Der Medikationsplan soll u. a. eine Überprüfung von Arzneimittelinteraktionen bei Polypharmazie erlauben.

\section{Kurz wirksame versus lang wirksame Präparate}

Modifiziert 2019. Konsensbasierte Empfehlung: Präparate mit retardierter $\mathrm{Ga}$ lenik bzw. langer Wirkdauer sollten eingesetzt werden. Starker Konsens (100\%; $100 \%)$

Kommentar: Für die Einnahme von retardierten Präparaten nach einem festen Einnahmeschema werden eine bessere Schmerzkontrolle und Therapieadhärenz und ein niedrigeres Risiko für Stürze und Suchtentwicklung angenommen. Diese Annahmen sind jedoch nicht durch methodisch hochwertige Studien belegt. Bei der Entscheidung für ein kurz oder ein lang wirksames opioidhaltiges Analgetikum sollen die individuelle Wirksamkeit und Verträglichkeit der jeweiligen Galeniken berücksichtigt werden [125].
Möglicherweise ist die regelmäßige Einnahme der Opioide relevanter als ihre Galenik [40].

\section{Einnahmeschema}

Bestätigt 2019. Konsensbasierte Empfehlung: Die Einnahme der opioidhaltigen Analgetika sollte nach einem festen Zeitplan (in Abhängigkeit von der Wirkungsdauer des jeweiligen Präparats) erfolgen. Starke Empfehlung. Starker Konsens

\section{Präparateaustausch}

Bestätigt 2019. Konsensbasierte Empfehlung: Bei stabiler Einstellung sollte ein Umsetzen auf ein Präparat mit anderen pharmakokinetischen und-dynamischen Charakteristika nur in Rücksprache mit dem behandelnden Arzt und nach Aufklärung des Patienten erfolgen. Starke Empfehlung. Starker Konsens

Kommentar: Aus Sicht der Patientenvertreterinnen der Leitliniengruppen soll bei stabiler Einstellung ein Umsetzen auf ein Austauschpräparat nur in Rücksprache zwischen dem behandelnden Arzt und dem Apotheker und nach Aufklärung des Patienten erfolgen. Nur wenn der behandelnde Arzt einem Austausch ausdrücklich zustimmt, darf dieser vorgenommen werden.

\section{Titration}

Bestätigt 2019. Konsensbasierte Empfehlung: Die Therapie soll mit niedrigen Dosen begonnen werden. Starke Empfehlung. Starker Konsens

\section{Titration}

Bestätigt 2019. Konsensbasierte Empfehlung: In Abhängigkeit von Wirksamkeit und Verträglichkeit soll die Dosis schrittweise gesteigert werden, um die individuellen Therapieziele $z u$ erreichen. Starke Empfehlung. Starker Konsens

\section{Bedarfsmedikation mit nicht-retardierten opioidhaltigen Analgetika in der Einstellungsphase}

Redaktionelle Änderung 2019. Konsensbasierte Empfehlung: In der Einstellungsphase kann eine Bedarfsmedikation mit nicht-retardierten oralen opioidhaltigen Analgetika oder nicht-opioidhaltigen Analgetika zur Findung der optimalen 
Dosis eingesetzt werden. Offene Empfehlung. Starker Konsens

\section{Ultrakurz wirksame bukkal oder nasal wirksame Opioide}

Neu 2019. Konsensbasierte Empfehlung: Ultrakurz wirksame bukkal oder nasal wirksame Opioide sollen nicht als Bedarfsmedikation empfohlen werden. Konsens (94\%; 94\%)

Kommentar: Es liegen keine RCTs vor. Aufgrund klinischer Erfahrungen sieht die Leitliniengruppe ein relevantes Risiko missbräuchlicher Verwendung ultrakurz wirksamer Opioide bei CNTS. Auch liegt für den CNTS keine Zulassung vor.

\section{Therapieresponder und optimale Dosis}

Bestätigt 2019. Konsensbasierte Feststellung: Eine optimale Dosis liegt bei einem Erreichen der zuvor formulierten Therapieziele bei gleichzeitigen geringen bzw. tolerablen Nebenwirkungen vor. Starker Konsens

Kommentar: Ob ein Ansprechen (Erreichen der individuellen Therapieziele) auf die und eine ausreichende Verträglichkeit der Therapie (= Therapieresponse) vorliegen, lässt sich nach der klinischen Erfahrung der Leitliniengruppe nach 4-6 Wochen beurteilen.

Eine Therapieresponse aus medizinischer Sicht sind eine Verbesserung der Funktionsfähigkeit im Alltag (z.B. Arbeitswiederaufnahme, „wieder Rasen mähen können") und/oder eine mindestens $30 \%$ ige Schmerzreduktion. Ein gutes Ansprechen (= „Therapieresponse") zeichnet sich im weiteren Verlauf durch eine fehlende bzw. nur geringe Toleranzentwicklung sowie keine oder eine nur geringe Dosissteigerung in einem mehrmonatigen Zeitraum aus.

\section{Höchstdosis}

Bestätigt 2019. Redaktionelle Änderung: Evidenzbasierte Empfehlung: Eine Dosis von $>120 \mathrm{mg} / \mathrm{Tag}$ oralem Morphinäquivalent soll nur in Ausnahmefällen überschritten werden. Starke Empfehlung. Starker Konsens

Kommentar modifiziert 2019: Die Tagesdosen in dem SR von Longterm-open-label-Studien von RCTs waren wie folgt: Buprenorphin transder- mal $(5-40 \mu \mathrm{g} / \mathrm{h}$; Durchschnitt $14 \mu \mathrm{g} / \mathrm{h})$; Hydromorphon (8-32 mg/d; Durchschnitt $17 \mathrm{mg} / \mathrm{d}$ ); Morphin (Maximum $90 \mathrm{mg} / \mathrm{d}$, die Hälfte der Patienten benutzte $60 \mathrm{mg} / \mathrm{d}$ ); Oxycodon: $20-140 \mathrm{mg} / \mathrm{d}$ (mittlere Dosis in einer Studie $44 \mathrm{mg} / \mathrm{d}$ ); Oxycodon/Naloxon (maximale Dosis $120 / 60 \mathrm{mg} / \mathrm{d}$; durchschnittliche Dosis 44/22 mg/d); Tapentadol (100-500 mg/d; durchschnittliche Dosis $368 \mathrm{mg} / \mathrm{d}$ ) [12]. Bei einer einjährigen nicht verblindeten Vergleichsstudie lagen die durchschnittlichen Enddosen von transdermalem Fentanyl bei $50 \mu \mathrm{g} / \mathrm{h}$ und von oralem Morphin bei $80 \mathrm{mg} / \mathrm{d}$ [2].

Eine retrospektive Kohortenstudie aus den USA (Medicaid-Patienten) zeigte einen Anstieg von Todesfällen in Abhängigkeit von der Opioiddosis bei mindestens einer Opioidverschreibung: 1-19 mg/d: „adjusted“ (aHR 5,6 [1,6; $19,3]) ; 20-49$ mg/d (aHR 6,2; [1,9-20,0]), $50-89 \mathrm{mg} / \mathrm{d} \quad$ (aHR 11,9; [3,7-38,8]), 90-119 mg/d (aHR 15,8; [4,6-53,9]) und $>120 \mathrm{mg} / \mathrm{d}$ (aHR 20,3; [6,4-64,3]) [76].

In einer retrospektiven Querschnittsstudie im Jahr 2014 mit 4.028.618 Versicherten von 69 gesetzlichen Krankenversicherungen waren Tagesdosen $>120 \mathrm{mg}$ MEQ mit mehr Krankenhausaufenthalten mit Diagnosen von psychischen und Verhaltensstörungen wegen Alkohol, Opioiden, Tranquilizern und multiplen Substanzen sowie Intoxikationen mit Narkotika assoziiert (1,6\% vs. 2,9\%) als Behandlungen mit Tagesdosen $<120 \mathrm{mg}$ MEQ [83].

Aktuelle internationale Leitlinien empfehlen folgende Höchstdosen bzw. Dosen, bei denen eine Reevaluation notwendig ist: Frankreich: $>120 \mathrm{mg}$ MEQ/d (Hinzuziehen eines Spezialisten bei $>150 \mathrm{mg}$ MEQ/d) [103] sowie USA und Kanada: $>90 \mathrm{MEQ} / \mathrm{d}[21,59]$.

\section{9. Überschreiten der empfohlenen Höchstdosen}

Bestätigt 2019. Konsensbasierte Empfehlung: Vor Erhöhungen der Dosis auf $>120 \mathrm{mg} / \mathrm{d}$ orales Morphinäquivalent sollen erneut die Indikation einer Therapie mit opioidhaltigen Analgetika sowie anderer Therapieoptionen und mögliche missbräuchliche Verwendung der rezep- tierten Medikamente überprüft werden. Starke Empfehlung. Starker Konsens

Kommentar ergänzt 2019: Vor einer Dosiserhöhung $>120 \mathrm{mg} / \mathrm{d}$ orales Morphinäquivalent ist $\mathrm{zu}$ überprüfen: Liegt eine relevante Toleranzentwicklung vor? Gibt es Hinweise für die Entwicklung einer Opioidabhängigkeit? Liegen andere Hinweise für eine mögliche missbräuchliche Verwendung der rezeptierten Medikamente vor? Ist der/die Patient/in ggf. mit einem Opioidauslass-/ Reduktionsversuch einverstanden? Gibt es Therapiealternativen?

Andererseits sind pharmakogenetische Unterschiede im Ansprechen auf Opioide zu berücksichtigen [119].

\section{Langzeitanwendung von Opioiden}

Bestätigt 2019. Konsensbasierte Empfehlung: Eine Therapie $>3$ Monate soll nur bei Therapierespondern durchgeführt werden. Starke Empfehlung. Starker Konsens

\section{Bedarfsmedikation mit opioidhaltigen Analgetika - Langzeittherapie}

Bestätigt 2019. Konsensbasierte Empfehlung: In der Langzeittherapie sollte eine Bedarfsmedikation mit opioidhaltigen Analgetika nicht durchgeführt werden. Empfehlung. Starker Konsens

Kommentar ergänzt: In Einzelfällen (z.B. geplante größere körperliche Belastung bei chronischem Arthroseschmerz) kann eine einmalige prophylaktische Erhöhung der Dosis eines retardierten opioidhaltigen Analgetikums und/oder die bedarfsweise Einnahme eines kurz wirksamen opioidhaltigen Analgetikums bzw. eines Nichtopioids erwogen werden.

\section{Behandlung von Übelkeit und Erbrechen}

Bestätigt 2019. Konsensbasierte Empfehlungen: Eine antiemetische Behandlung kann bereits zu Beginn der Therapie erfolgen. Offene Empfehlung. Nach etwa 2-4 Wochen soll die Indikation für ein Absetzen der antiemetischen Therapie überprüft werden. Starke Empfehlung. Starker Konsens

Kommentar: Bei den meisten Patienten entwickelt sich nach 2-4 Wochen eine 
Toleranz gegenüber der emetischen Wirkung von opioidhaltigen Analgetika, eine routinemäßige Gabe von Antiemetika ist nicht notwendig [142].

Nach der klinischen Erfahrung der Steuergruppe kann die emetische Wirkung von Opioiden unterschiedlich sein. Mögliche Gründe für das unterschiedliche Ansprechen sind genetische, physiologische sowie pharmakinetische und -dynamische Faktoren.

\section{Praxiswerkzeug}

- Antiemetika bei opioidinduzierter Übelkeit

https://www.awmf.org/leitlinien/ detail/ll/145-003.html

\section{Behandlung von Obstipation} Redaktionelle Änderung 2019. Konsensbasierte Empfehlung: Die Behandlung von Obstipation mit Laxanzien sollte bei den meisten Patienten prophylaktisch begonnen werden. Empfehlung. Starker Konsens

Kommentar: Eine Entscheidung über eine prophylaktische oder eine bedarfsweise durchgeführte Laxanzientherapie ist im Einzelfall und in Abhängigkeit vom Defäkationsmuster des Patienten zu treffen. Bei Patienten mit einer vorbestehenden Obstipation/Obstipationsneigung ist die prophylaktische Gabe von Laxanzien sinnvoll [105]. Bei vielen Patienten kann während der gesamten Therapiedauer mit opioidhaltigen Analgetika die Gabe von Laxanzien erforderlich sein.

Nach der klinischen Erfahrung der Steuergruppe kann die obstipationsfördernde Wirkung von Opioiden unterschiedlich sein. Mögliche Gründe für das unterschiedliche Ansprechen sind genetische, physiologische sowie pharmakinetische und -dynamische Faktoren.

\section{Auswahl von Laxanzien}

Neu 2019. Evidenzbasierte Feststellung: Die Bevorzugung eines Medikaments zur Prophylaxe und Therapie der opioidinduzierten Obstipation ist aufgrund der aktuellen Studienlage nicht möglich. Starker Konsens bzw. Konsens (96\%; $93 \%$ )

Kommentar: Es gibt nur eine kontrollierte Studie zum Einsatz von konventionellen Laxanzien bei der opioidinduzierten Obstipation (OIC) beim chronischen
Nichttumorschmerz: In einer randomisierten, doppelblinden, placebokontrollierten Studie konnte die Überlegenheit von Macrogol gegenüber Placebo bei der Behandlung einer methadoninduzierten Obstipation gezeigt werden [73].

Studien zu peripheren Opioidantagonisten (PAMORA) zeigten die Wirksamkeit lediglich im Vergleich zu Placebo und nicht im Vergleich zu konventionellen Laxanzien. In den Studien zu Oxycodon/ Naloxon erfolgte ein Vergleich mit Oxycodon, jedoch kein Vergleich mit Oxycodon und einem konventionellen Laxans oder anderen Opioiden [104]. Es ist derzeit nicht eindeutig belegt, ob PAMORA klinisch gegenüber den etablierten (wenngleich in dieser Anwendung kaum evidenzgestützten) und kostengünstigeren Laxanzien in der Behandlung der OIC Vorteile bieten (bei deutlich höheren Kosten). Eine abschließende Bewertung des Stellenwerts im Vergleich zu konventionellen Laxanzien ist daher derzeit nicht möglich.

Es wurden verschiedene PAMORA in klinischen Studien bei opioidinduzierter Obstipation (OIC) (mit und ohne zugrunde liegende Tumorerkrankung) untersucht. Eine Metaanalyse aus dem Jahr 2013 analysierte 14 randomisierte, kontrollierte Studien (RCT) mit insgesamt 4101 Patienten [69]. Dabei waren die PAMORA signifikant besser als Placebo in der Behandlung der OIC (relatives Risiko [RR] für ein Therapieversagen: $0,69[0,63,0,75])$, während die analgetische Wirksamkeit der Opioidbehandlung nicht verändert war. Auch die untersuchten Einzelsubstanzen zeigten jeweils eine Überlegenheit gegenüber Placebo: Methylnaltrexon (6 RCT, 1610 Patienten, RR 0,66 [0,54, 0,84]), Alvimopan (4 RCT, 1693 Patienten, RR $0,71[0,65,0,78])$ und orales Naloxon (4 RCT, 798 Patienten, RR 0,64 [0,56, $0,72]$ ). In der gepoolten Analyse waren die Gesamtzahl von unerwünschten Ereignissen sowie Durchfall und Bauchschmerzen signifikant häufiger bei den PAMORA im Vergleich zu Placebo. Naloxegol ist ein neuer PAMORA, der noch nicht von der o.g. Metaanalyse erfasst war und der in Deutschland zugelassen worden ist zur Behandlung der OIC bei erwachsenen Patienten, die unzu- reichend auf Laxanzien angesprochen haben. Zwei große Phase-III-Studien belegten eine überlegene Wirksamkeit von Naloxegol gegenüber Placebo ohne Verminderung der analgetischen Wirkung. Für die 25-mg-Dosierung ergab sich in einer Studie der ITT-Population eine Ansprechrate von 44,4\% (RR für ein Therapieansprechen von 1,51 $[1,17,1,95])$ und in der Subgruppe der laxanzienrefraktären Patienten (LIRGruppe) eine Ansprechrate von 48,7\% (RR für ein Therapieansprechen von 1,69 $[1,21,2,37])$. Unerwünschte Wirkungen waren Durchfall $(0,8 \%)$, Bauchschmerzen $(1,9 \%)$ und Oberbauchschmerzen $(1,9 \%)[5,69]$.

Vergleichsstudien zwischen Opioidantagonisten und Laxanzien sind rar und beziehen sich ebenso auf Tumorschmerzbzw. Palliativpatienten. In der Übersicht von Bader et al. [8] konnten keine Unterschiede der Effektivität von oralem Naloxon gegenüber verschiedenen Laxanzien gefunden werden.

Methylnaltrexon subkutan ist in Deutschland zur Therapie opioidinduzierter Obstipation bei chronischen nicht-tumorbedingten Schmerzen zugelassen, die auf Standardlaxanzien nicht ausreichend ansprechen. Orales Methylnaltrexon ist in Deutschland aktuell nicht zugelassen. Naloxegol ist nur zugelassen bei Erwachsenen mit OIC, die unzureichend auf Laxanzien angesprochen haben. Naloxon liegt in Deutschland nur zur Injektion bei opioidbedingter Atemdepression vor.

Hinsichtlich des Einsatzes von Tilidin/ Naloxon liegen nur Daten einer deutschen Fallserie vor, in der bei 4/335 Patienten im Laufe von 2 Jahren eine Obstipation als Nebenwirkung dokumentiert wurde [66].

Oxycodon/Naloxon ist in Bezug auf Häufigkeit und Intensität der Obstipation Oxycodon allein überlegen [60], ein Vergleich mit anderen Opioiden liegt für die Kombination in Form randomisierter Studien nicht vor. Trotz mangelhafter Evidenzlage kann die Kombination von oralem Oxycodon mit Naloxon nach Ansicht der Leitliniengruppe im Falle einer OIC alternativ in Stufe 1 der Obstipationstherapie gewählt werden. 
Ein Cochrane-Review fand eine bessere gastrointestinale Verträglichkeit von Tapentadol im Vergleich zu Oxycodon bei chronischen muskuloskeletalen Schmerzen [131]. Ein Cochrane-Review zu transdermalem Fentanyl bei Krebsschmerzen zeigte eine Verringerung der OIC im Vergleich zu oralem retardiertem Morphin [79]. Eine Opioidrotation ist bei den in den Cochrane-Reviews untersuchten Vergleichen eine Option zur Therapie der OIC.

Im Tumorschmerzbereich bzw. in der Palliativmedizin wird die Prophylaxe und Therapie einer Obstipation im Rahmen eines Stufenschemas empfohlen [55]. Es handelt sich um eine konsensbasierte Empfehlung, nach der ein standardisiertes Vorgehen in Form eines Stufenplans gewählt werden sollte.

\section{Praxiswerkzeug}

- Laxanzien bei opioidinduzierter Obstipation

https://www.awmf.org/leitlinien/ detail/1l/145-003.html

\section{Vorgehen bei Wirkungs- abnahme}

Bestätigt 2019. Konsensbasierte Empfehlung: Bei einer Wirkungsabnahme unter einer Langzeittherapie mit Opioiden soll eine Reevaluation durchgeführt werden. Starke Empfehlung. Starker Konsens

Kommentar:

\section{Differenzialdiagnosen bei Wirkungsabnahme}

- Krankheitsprogression

- Toleranzentwicklung

- Opioidinduzierte Hyperalgesie

- Fehlgebrauch, Missbrauch oder eine Substanzabhängigkeit (siehe 54.)

\section{Krankheitsprogression}

Bei manchen Erkrankungen kann es (ähnlich wie bei einer Tumorerkrankung) zu einer Verschlechterung des Krankheitsbilds kommen, die wiederum zu einer Zunahme der Nozizeption führt. So können z. B. bei einer Arthrose mit fortschreitendem Verschleiß des Gelenkknorpels die bewegungsabhängigen Schmerzen massiv verstärkt werden.

\section{Toleranz}

Im Rahmen der Dauertherapie (z. B. mit Opioiden) kann es zu einer Minderung der analgetischen Wirksamkeit kommen. Als Ursache wird eine Wanderung von Opioidrezeptoren von der Zelloberfläche in das Zellinnere vermutet (Rezeptorinternalisation), wo sie nicht mehr für die analgetische Wirkung zur Verfügung stehen.

\section{Opioidinduzierte Hyperalgesie}

In Einzelfällen wurde bei einer Opioidtherapie über neurotoxische $\mathrm{Ne}$ benwirkungen berichtet, die als $\mathrm{Hy}$ peralgesie, manchmal mit anderen neurologischen (Allodynie, Myoklonien) und psychiatrischen (Halluzinationen, Albträume) Symptomen, auftreten.

\section{Toleranzentwicklung}

Bestätigt 2019. Konsensbasierte Empfehlung: Bei einer Toleranzentwicklung kann eine Dosiserhöhung, ein Opioidwechsel oder ein Opioidentzug durchgeführt werden. Offene Empfehlung. Starker Konsens

Kommentar: Die Leitliniengruppe empfiehlt, dass in der Regel nicht mehr als 2 Opioidwechsel bei Toleranzentwicklung durchgeführt werden. Eine anhaltende Toleranzentwicklung mit wiederholtem Bedarf für eine Dosisanpassung auch nach Opioidwechsel ist zum Erhalt einer klinisch relevanten Wirkung (Responder) nicht sinnvoll. Statt einer weiteren Opioidrotation sollte eine schrittweise Opioidreduktion bzw. ein Opioidentzug durchgeführt werden.

\section{Praxiswerkzeug}

- Opioidrotation

https://www.awmf.org/leitlinien/ detail/1l/145-003.html

\section{Opioidinduzierte Hyperalgesie} Bestätigt 2019. Konsensbasierte Empfehlung: Bei Hinweisen auf die Entwicklung einer opioidinduzierten Hyperalgesie soll eine schrittweise Opioidreduktion und/ oder ein Opioidentzug durchgeführt werden. Starke Empfehlung. Starker Konsens

Kommentar: Weiterführende Literatur [95]. Eine Opioidreduktion kann auch durch eine zeitgleiche Opioidrotation mit Reduktion (z.B. 50\%) der errechneten Äquivalenzdosis erreicht werden.

\section{Therapieüberwachung und Dokumentation}

\section{Regelmäßige Therapieüberwa- chung}

Bestätigt 2019. Konsensbasierte Empfehlung: Bei einer Langzeittherapie mit Opioiden soll in regelmäßigen Abständen überprüft werden, ob die Therapieziele weiter erreicht werden und ob es Hinweise für Nebenwirkungen (z. B. Libidoverlust, psychische Veränderungen wie Interessenverlust, Merkfähigkeitsstörungen sowie Sturzereignisse) oder für einen Fehlgebrauch der rezeptierten Medikamente gibt. Starke Empfehlung. Starker Konsens

Kommentar: Die Leitliniengruppe empfiehlt eine Therapiekontrolle in der Einstellungsphase mindestens alle 4 Wochen und in der Langzeittherapie mindestens einmal im Quartal.

\section{Therapiedokumentation}

Bestätigt 2019. Konsensbasierte Empfehlung: Die Behandlung soll regelmäßig dokumentiert werden. Starke Empfehlung. Starker Konsens

Kommentar: Inhalte der Therapiedokumentation sind:

a) Erreichte Schmerzlinderung (z.B. Schmerztagebuch)

b) Funktionsniveau und Lebensqualität; besonders engmaschig in der Einstellungsphase

c) Entwicklung eines abweichenden substanzbezogenen Verhaltens

d) Unzulässige Weitergabe oder Beschaffung opioidhaltiger Analgetika

e) Umfassende Abschätzung der Schmerzlinderung, Verbesserung der Lebensqualität und des Funktionsniveaus und der daraus resultierenden verhaltensmäßigen, emotionalen und kognitiven Veränderungen

\section{Praxiswerkzeug}

- Verlaufsdokumentation BPI https://www.dgpalliativmedizin. de/images/stories/pdf/ag/ag_ forschungBPI-Kurzform.pdf

\section{Urinkontrollen auf Drogen}

Neu 2019. Konsensbasierte Empfehlung: Urinkontrollen auf Drogen sollen nicht routinemäßig, jedoch vor und während 
einer Langzeittherapie mit Opioiden bei Verdacht auf missbräuchliche Verwendung von Drogen und/oder rezeptpflichtiger Medikamente durchgeführt werden. Starke Empfehlung. Starker Konsens (100\%; $100 \%)$

Kommentar: Die US-amerikanische Leitlinie empfiehlt Urinuntersuchungen auf Drogen bei allen Patienten vor Einleitung einer Opioidtherapie („should“). Mindestens jährliche Kontrollen sind $\mathrm{zu}$ erwägen [59]. In der kanadischen Leitlinie werden beide Maßnahmen fakultativ empfohlen [21]. In Anbetracht der fehlenden Belege für positive Effekte eines routinemäßigen Drogenscreenings wird dieses nur bei klinischem Verdacht empfohlen.

\section{Beendigung einer Therapie mit opioidhaltigen Analgetika}

\section{Beendigung eines Therapieversuchs}

Bestätigt 2019. Konsensbasierte Empfehlung: Wenn in der Einstellungsphase (maximal 12 Wochen) die individuellen Therapieziele nicht erreicht werden bzw. (aus Patienten- und/oder Arztsicht) nicht ausreichend therapiebare bzw. nicht tolerierbare Nebenwirkungen auftreten, soll die Therapie mit opioidhaltigen Analgetika schrittweise beendet werden. Starke Empfehlung. Starker Konsens

\section{Beendigung einer Therapie $>12$ Wochen}

\section{Konsensbasierte Empfehlungen} Bestätigt 2019. a. Wenn die individuellen Therapieziele nicht mehr erreicht werden bzw. (aus Patienten- und/oder Arztsicht) nicht ausreichend therapiebare bzw. nicht tolerierbare Nebenwirkungen auftreten, soll die Therapie mit opioidhaltigen Analgetika schrittweise beendet werden. Starke Empfehlung. Starker Konsens

Bestätigt 2019. b. Wenn die individuellen Therapieziele durch andere medizinische Maßnahmen (z. B. Op., Bestrahlung, ausreichende Behandlung des Grundleidens) oder physiotherapeutische oder physikalische oder psycho- therapeutische Maßnahmen erreicht sind, soll die Therapie mit opioidhaltigen Analgetika schrittweise beendet werden. Starke Empfehlung. Starker Konsens

Bestätigt 2019. c. Wenn der Patient die rezeptierten opioidhaltigen Analgetika trotz Mitbehandlung durch einen Suchtspezialisten missbräuchlich verwendet, soll die Therapie mit opioidhaltigen Analgetika schrittweise beendet werden. Starke Empfehlung. Starker Konsens

\section{Medikamentenpause}

Bestätigt 2019. Nach sechs Monaten soll mit Patienten mit einer Therapieresponse die Möglichkeit einer Dosisreduktion und/oder eines Auslassversuchs besprochen werden, um die Indikation der Fortführung der Behandlung und das Ansprechen auf parallel eingeleitete nicht-medikamentöse Therapiemaßnahmen (z.B. multimodale Therapie) zu überprüfen. Starke Empfehlung. Starker Konsens

Kommentar: Die Gründe für diese Empfehlungen sind:

a) Eine spontane Symptombesserung (natürlicher Krankheitsverlauf) ist bei einigen CNTS-Syndromen, z. B. chronischen Rückenschmerzen [88] und Postzosterneuralgie [127], beschrieben. Alternativ könnte eine funktionell relevante Analgesie mittelfristig über Verbesserung der Funktionalität zu einer klinischen Gesamtverbesserung führen.

b) Es ist möglich, dass die Therapieziele durch parallel eingeleitete physikalische Therapien und/oder psychotherapeutische Verfahren erreicht werden können und ein opioidhaltiges Analgetikum nicht mehr erforderlich ist.

c) Randomisierte, placebokontrollierte Studien beim CNTS wurden maximal bis 24 Wochen durchgeführt.

Kohortenstudien, auf die eine Empfehlung zum Intervall der Reduktionsversuche und „drug holidays" gestützt werden kann, wurden nicht gefunden.
44. Opioidassoziierte psychische Auffälligkeiten

Redaktionelle Änderung 2019. Konsensbasierte Empfehlung: Wenn der Patient opioidassoziierte psychische Auffälligkeiten zeigt, sollen folgende Optionen erwogen werden:

- Reduktion der Opioiddosis

- Opioidwechsel

- Schrittweise Beendigung der Therapie mit opioidhaltigen Analgetika.

Starke Empfehlung. Starker Konsens Kommentar: Bei der Differenzialdiagnostik psychischer Auffälligkeiten unter einer Therapie mit opioidhaltigen Analgetika (z.B. Delir ohne Demenz bei geriatrischen Patienten) sind mögliche zentralnervöse Nebenwirkungen anderer Medikamente sowie somatische Krankheitsfaktoren (z. B. Fieber, Exsikkose) zu berücksichtigen.

Hinweise für opioidassoziierte psychische Auffälligkeiten können sein:

- (Selbst-)gefährdendes Verhalten;

Konsum illegaler Drogen oder von Alkohol; intentionale Überdosierung oder Suizidversuch mit opioidhaltigen Analgetika; aggressive, bedrohliche oder streitlustige Verhaltensweisen

- Halluzinationen

- Verwirrtheit

- Zunahme von vorbestehenden oder Neuauftreten von Angst- und depressiven Symptomen

- Delir ohne Demenz

- Verschlechterung einer bestehenden Demenz

\section{Opioidentzug als therapeuti- sche Maßnahme}

Bestätigt 2019. Evidenzbasierte Empfehlung: Bei Patienten mit persistierenden Schmerzen und/oder Beeinträchtigungen/Nebenwirkungen unter langfristiger Einnahme von opioidhaltigen Analgetika kann ein Opioidentzug innerhalb eines multimodalen Therapieprogramms als therapeutische Maßnahme erwogen werden. EL4b. Starker Konsens

Kommentar: Es wurden keine neuen Kohortenstudien aus deutschen Schmerzzentren gefunden, welche die in früheren Studien beschriebene Reduktion von Schmerzen und Beeinträchti- 


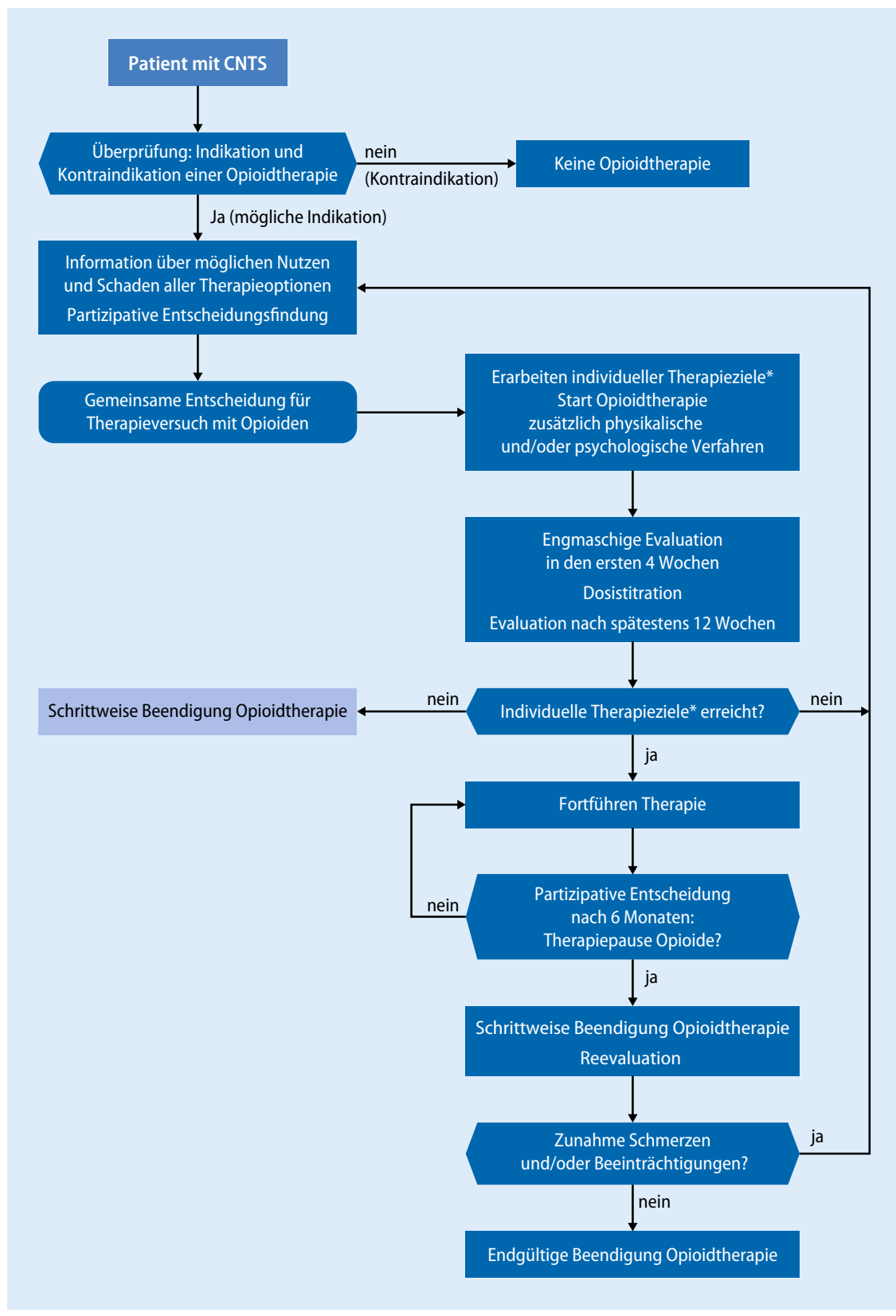

Abb. 1 A Algorithmus der Therapie mit opioidhaltigen Analgetika bei chronischen nicht-tumorbedingten Schmerzen (CNTS). Sternchen: z. B. $30 \%$ Schmerzreduktion und/oder Verbesserung der Funktionsfähigkeit im Alltag bei vom Patienten und Arzt tolerablen Nebenwirkungen; eine weitere mögliche Indikation für eine Beendigung einer Opioidtherapie ist eine missbräuchliche Verwendung der rezeptierten Opioide

gungserleben durch einen Opioidentzug innerhalb eines multimodalen Therapieprogramms nachwiesen [150].

\section{Stationäre Opioidreduktion}

Neu 2019. Konsensbasierte Empfehlung: Wenn die Opioidtherapie unter ambulanten und/oder teilstationären Bedingungen nicht reduziert bzw. beendet werden kann, soll eine stationäre Medikamentenreduk-
Begleittherapien inkl. Selbstmanagementtraining sollen erwogen werden. Über Angebote der Selbsthilfe soll informiert werden. Starker Konsens

Kommentar: Bei Beendigung einer Anwendung opioidhaltiger Analgetika können Entzugssymptome auftreten. Vor dem Absetzen sollte die Evaluation von Behandlung, Komorbiditäten, psychischer Verfassung und anderer relevanter Faktoren abgeschlossen sein. Der Patient und seine Familie sollten vorher über die Vorgehensweise während der Ausleitung und häufig auftretende Entzugssymptome unterrichtet werden. Der Kontakt zum Patienten sollte bis zum Abschluss des Absetzens aufrechterhalten werden; eine zusätzliche psychotherapeutische Unterstützung kann im Einzelfall sinnvoll sein.

Die Maßnahmen und Entscheidungsprozesse bei der Indikationsstellung, Durchführung und möglichen Beendigung einer Therapie mit opioidhaltigen Analgetika sind in einem Algorithmus (siehe - Abb. 1) zusammengefasst.

\section{E. Spezielle Patientengruppen}

\section{8. Ältere Patienten}

Bestätigt 2019. Konsensbasierte Empfehlung: Die Therapie soll mit einer niedrigen Dosis begonnen werden. Dosissteigerungen sollen langsam durchgeführt werden. Engmaschige Kontrollen von Wirksamkeit und Verträglichkeit sollen erfolgen. Starke Empfehlung. Starker Konsens

Kommentar modifiziert 2019: Altersbedingte pharmakodynamische und pharmakokinetische Veränderungen führen zu einer längeren Wirkdauer der Opioide im Alter. U.a. aus diesen Gründen wird empfohlen, zu Beginn einer Opioidtherapie mit einer ungefähr 25-50\%igen Dosisreduktion im Vergleich zu Jüngeren zu beginnen und langsamer zu steigern [21].

Post-hoc-Analysen von RCTs, die von den Herstellern der Medikamente finanziert wurden, zeigten eine Überlegenheit von Oxycodon/Naloxon deterrent (in Deutschland nicht zugelassen) gegen Placebo/Naloxon deterrent bei Patienten $>65$ Jahre mit chronischem Rückenschmerz [91] und von retardiertem Tapentadol bei Patienten $>75$ Jahre

\section{Praxis der Beendigung der Therapie \\ Modifiziert 2019. Konsensbasierte Emp- fehlung: Eine Langzeitanwendung mit opioidhaltigen Analgetika soll schrittweise beendet werden. Medikamentöse, physio- therapeutische und psychotherapeutische}


mit chronischem Rücken- oder Knieschmerz gegenüber Oxycodon [13]. Eine nicht-verblindete und nicht-kontrollierte Studie mit Hydrocodon bei 24 Patienten $\geq 75$ Jahren und nicht-neuropathischem CNTS fand eine klinisch relevante Reduktion von Schmerz- und Beeinträchtigungserleben bei den Patienten, welche die Studie beendeten. Die Abbruchrate wegen Nebenwirkungen lag bei $30 \%$ [14].

Eine systematische Übersichtsarbeit von 23 placebokontrollierten RCTs mit Patientenpopulationen mit chronischen muskuloskeletalen Schmerzen und einem Durchschnittsalter $>60$ Jahre fand geringe durchschnittliche Effekte auf Schmerz und Beeinträchtigungserleben. Die Abbruchraten mit Opioiden waren viermal höher als mit anderen Analgetika [102].

\section{Kinder und Jugendliche}

Redaktionelle Änderung 2019. Konsensbasierte Empfehlungen: Eine Therapie mit opioidhaltigen Analgetika soll nur in Ausnahmefällen durchgeführt werden. Starke Empfehlung. Starker Konsens

Falls eine begründete Entscheidung für einen individuellen Therapieversuch mit Opioiden getroffen wurde, sollte dieser durch spezialisierte Zentren/Kinderärzte durchgeführt werden. Empfehlung. Starker Konsens

\section{Schwangere}

Redaktionelle Änderung 2019. Konsensbasierte Empfehlungen: Die Beendigung einer Therapie mit opioidhaltigen Analgetika soll für den Fall einer geplanten Schwangerschaft dringend angeraten werden. Starke Empfehlung. Starker Konsens

Wird unter einer laufenden Therapie mit opioidhaltigen Analgetika eine Schwangerschaft festgestellt, sollte die Beendigung der Therapie mit opioidhaltigen Analgetika angestrebt werden. Empfehlung. Starker Konsens

Kommentar: Alternativen für Opioide in der Schwangerschaft sind in erster Linie nicht-medikamentöse Verfahren der Schmerztherapie. Nichtopioide (NSAR und stärker eingeschränkt Metamizol) sind bei der Anwendung in der Schwangerschaft auf die ersten beiden Trimena (bis zu 28. SSW) begrenzt. Auch die Gabe einiger Koanalgetika (Antidepressiva und Antikonvulsiva) ist in der Schwangerschaft eingeschränkt. Nur Paracetamol ist in der Schwangerschaft in allen Trimena grundsätzlich erlaubt. Deshalb kann es aufgrund einer indizierten und nicht anders zu erzielenden Analgesie notwendig sein, die Therapie mit opioidhaltigen Analgetika in der Schwangerschaft fortzuführen. Die Entbindung sollte dann in einem Perinatalzentrum Stufe I/II erfolgen, da bei dem Neugeborenen postpartal Entzugssymptome möglich sind. Für alle Opioide wird in www.embryotox.de über unzureichende Erfahrungen berichtet, zur Behandlung in der Schwangerschaft werden Morphin, Tramadol, Buprenorphin (50-300 systematisch ausgewertete Schwangerschaften) am ehesten empfohlen, ohne grundsätzliche Bedenken gegen andere Opioide (Fentanyl, Oxycodon, Hydromorphon). $\mathrm{Zu}$ Tilidin/Naloxon liegen nur geringe Erfahrungen ( $<50$ Schwangerschaften) und zu Tapentadol keine Erfahrungen vor [30]. Im Falle eines Absetzens sollen Entzugssymptome durch langsames Ausschleichen vermieden werden, da diese mit einem erhöhten Risiko von vorzeitiger Wehentätigkeit und Fehl- bzw. Frühgeburten einhergehen. Besteht ein schädlicher/abhängiger Gebrauch, sollte die Behandlung entsprechend Empfehlung 55 schmerzund suchttherapeutisch koordiniert und abgestimmt werden.

\section{Patienten mit schlafbezogenen Atmungsstörungen}

Neu 2019. Konsensbasierte Empfehlung: Die Indikation für eine Langzeitanwendung von Opioiden bei Patienten mit bekanntem obstruktivem Atemwegssyndrom soll streng gestellt werden. Wenn sich die Symptomatik eines Schlafapnoesyndroms trotz Optimierung der Atemtherapie unter einer Opioidtherapie verschlechtert, soll eine Reduktion bzw. ein Absetzen der Opioide empfohlen werden. Starke Empfehlung. Starker Konsens (100\%; 100\%)

Kommentar: Eine Langzeittherapie mit Opioiden erhöht das Risiko, ein zentrales Schlafapnoesyndrom zu entwickeln [124]. Eine systematische Über- sichtsarbeit fand Prävalenzraten von $24 \%$. Das Risiko steigt bei gleichzeitiger Einnahme von Tranquilizern/ Hypnotika und Morphinäquivalenzdosen von $\geq 200 \mathrm{mg} / \mathrm{d}$ [38]. Ein Opioidentzug [137] bessert die Symptomatik. Nach Fallberichten ist eine kontinuierliche Überdruckbeatmung in diesen Fällen oft ineffektiv bzw. kann das zentrale Schlafapnoesyndrom sogar verschlechtern. Eine Form der nichtinvasiven Heimbeatmung (druckkontrollierte Atmungsunterstützung, die es dem $\mathrm{Pa}$ tienten erlaubt, selbst zu atmen) konnte in einigen Untersuchungen bei Patienten unter Opioidtherapie das zentrale Schlafapnoesyndrom reduzieren [38].

\section{Patienten mit komorbiden psychischen Störungen}

Bestätigt 2019. Konsensbasierte Empfehlung: Die Therapie soll mit einer niedrigen Dosis begonnen werden. Dosissteigerungen sollen langsam durchgeführt werden. Engmaschige Kontrollen von Wirksamkeit und Verträglichkeit sollen erfolgen. Eine fachpsychotherapeutische Mitbehandlung soll erwogen werden. Starke Empfehlung. Starker Konsens

\section{Patienten mit aktueller Substanzabhängigkeit}

Bestätigt 2019 Konsensbasierte Empfehlung: Eine Therapie sollte nur in engmaschiger Absprache mit Ärzten und psychologischen Psychotherapeuten mit suchtmedizinischer Kompetenz durchgeführt werden. Empfehlung. Starker Konsens

Kommentar: Patienten mit einer möglichen Indikation für die Gabe von Opioiden aufgrund von Schmerzen und gleichzeitiger aktueller Substanzabhängigkeit (z. B. Alkohol, Amphetamine, Benzodiazepine, Cannabis, Kokain, Opiate) stellen eine interdisziplinäre Herausforderung dar, die eine enge Abstimmung bezüglich der Auswahl, Einleitung, Durchführung und Integration schmerz- und suchtmedizinischer Behandlungsoptionen notwendig macht. Ein alleiniger medikamentöser Therapieansatz ist hier in der Regel nicht indiziert. 


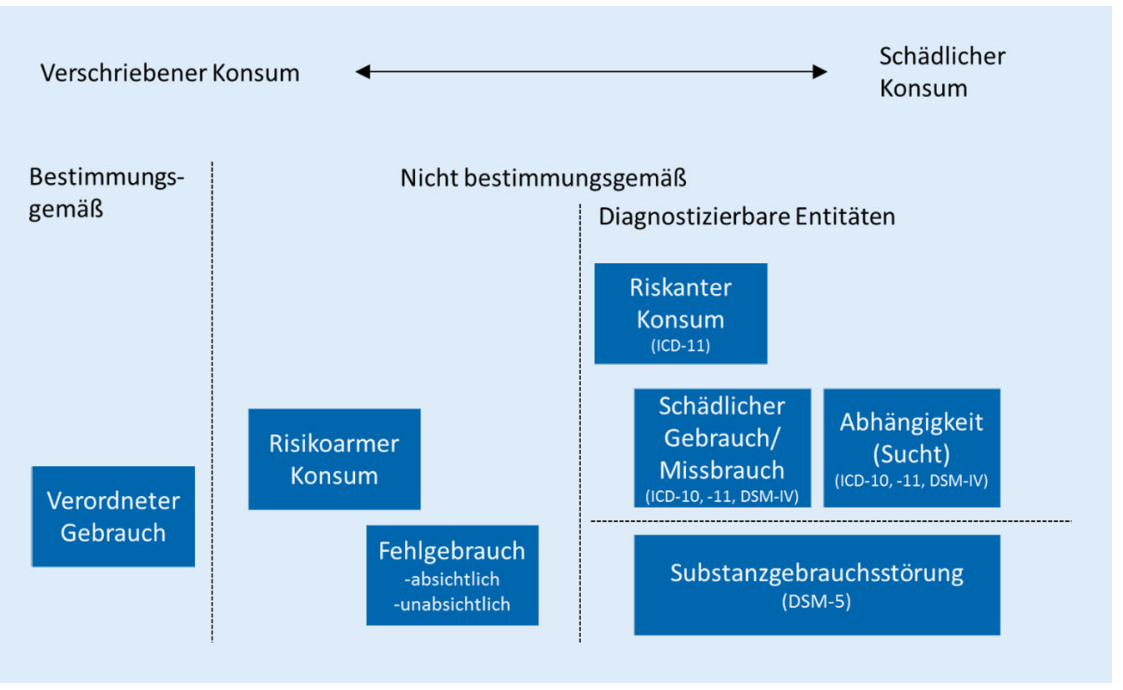

Abb. 2 ム Übersicht über Begrifflichkeiten

\section{Missbräuchliche Verwendung und Abhängigkeit von opioidhaltigen Schmerzmitteln, die aus medizinischer Indikation verschrieben wurden \\ Neu 2019: Vorwort: Begrifflichkeiten}

In der internationalen Literatur werden unterschiedliche Begriffe und diagnostische Kriterien für einen problematischen Umgang von Patienten und Ärzten mit Opioiden, welche aus therapeutischer Indikation verordnet werden, verwendet $[25,132]$. Die Bedeutung derselben Begriffe kann geografisch und kulturell unterschiedlich sein [135].

Im Folgenden werden die Ausführungen und Empfehlungen in der deutschen S3-Leitlinie zur Abhängigkeit von aus medizinischer Indikation verordneten Medikamenten dargestellt (• Abb. 2).

Bestimmungsgemäßer Konsum: Konsum von Substanzen gemäß den ärztlichen Empfehlungen und/oder den Vorgaben der Arzneimittelinformation, mit Einsatz der Substanz im Rahmen der zugelassenen Indikation und Dosierung.

Nicht-bestimmungsgemäßer Konsum (engl. „misuse"): Ausnahmen im Sinne eines nicht-bestimmungsgemäßen Gebrauchs sind möglich im Sinne

a) eines „off-label use“. Dieser bezeichnet den Einsatz eines Medikaments außerhalb der zugelassenen Indikation nach Aufklärung und Verordnung durch einen Arzt bzw. ärztlich verordnete Dosisanpassungen jenseits der zugelassenen Dosierung aufgrund individueller Gegebenheiten (z. B. beschleunigten enzymatischen Abbaus etc.).

b) eines „Medikationsfehlers“. Dieser liegt vor, wenn vom vorgegebenen Anwendungsschema - mit dem in Kauf genommenen Risiko eines Schadens für den Patienten - abgewichen wird.

c) eines „Fehlgebrauchs“. Dieser tritt ein, wenn ein Medikament intendiert anders eingesetzt wird (in Dosis oder Applikationsart), als es verordnet wurde.

Ein Fehlgebrauch kann in „unabsichtlich“ (z. B. Missverständnis in Bezug auf die Umsetzung einer verordneten Anwendung) und „absichtlich“ (z. B. für eine primär nicht intendierte Wirkung, wie Schlafinduktion durch Opioide) unterschieden werden.

Missbrauch (engl. „abuse“): übermäßiger, exzessiver, nicht-bestimmungsgemäßer Konsum von Substanzen mit Gesundheitsschäden in klinisch bedeutsamer Weise. Es fehlt entweder an einer medizinischen Indikation oder die vorgegebenen Rahmenbedingungen für den medizinischen Einsatz werden ignoriert. Ziel des Konsumenten ist häufig die Provokation psychotroper Effekte oder die Beseitigung von Entzugssymptomen.

Schädlicher Gebrauch (ICD-10): Ein Konsummuster, das lt. Definition der
ICD-10 physische oder psychische $\mathrm{Ge}$ sundheitsschäden, jedoch keine Abhängigkeit hervorruft.

ICD-11 ergänzt dies dahingehend, dass dieses Konsummuster während eines Monats kontinuierlich bzw. innerhalb eines Jahres episodisch aufgetreten sein muss und einen tatsächlichen Schaden der psychischen oder physischen Gesundheit der einnehmenden Person oder betroffener Dritter herbeigeführt hat.

Abhängigkeitssyndrom (ICD-10): Das Abhängigkeitssyndrom wird nach ICD10, ICD-11 oder DSM-IV kategorial, nach DSM-5 dimensional verstanden. Abhängigkeit ist in diesen Klassifikationssystemen übereinstimmend gekennzeichnet durch den 1) starken Konsumdrang, 2) Kontrollverlust, 3) Toleranzentwicklung und 4) Auftreten von körperlichen Entzugssymptomen, 5) Vernachlässigung anderer Interessen (Verpflichtungen) zugunsten des Substanzkonsums und 6) anhaltendem Substanzkonsum trotz des Nachweises eindeutiger schädlicher Folgen.

Entzugssyndrom (ICD-10): Zustand, der bei Absetzen oder Reduktion des Substanzkonsums auftritt und mit substanzspezifischen psychovegetativen Symptomen einhergeht. Ein Entzugssyndrom geht zurück, sobald dem Körper wieder die ursprüngliche oder eine ähnliche Substanz zugeführt wird. Das Entzugssyndrom ist häufig selbstlimitierend, tritt wenige Stunden nach der letzten Einnahme auf und kann über Tage bis Wochen anhalten.

Toleranz (Gewöhnung): Die Drogenwirkung lässt nach Phasen längeren Konsums nach. Toleranz entsteht durch a) eine neurologische Adaptation bzw. b) einen beschleunigten Abbau (verkürzte biologische Halbwertszeit) zugeführter Substanzen sowie c) psychovegetative Anpassungsvorgänge an die Substanzwirkung. Der Wirkverlust führt oft zu einer Dosissteigerung.

Körperliche Abhängigkeit: Körperliche Abhängigkeit erklärt das Auftreten eines Entzugssyndroms. Der Zustand tritt bei Absetzen oder Reduktion des Substanzkonsums auf und geht mit substanzspezifischen psychovegetativen Symptomen einher. Ein Entzugssyndrom geht zurück, 
sobald dem Körper wieder die ursprüngliche oder eine ähnliche Substanz zugeführt wird. Das Entzugssyndrom ist häufig selbstlimitierend, tritt wenige Stunden nach der letzten Einnahme auf und kann über Tage bis Wochen anhalten. Die körperliche Abhängigkeit wird mit einem Entzugssymptom gleichgesetzt, das sich dann ergibt, wenn die Dosis verringert oder im Verlauf eines Abstinenzversuchs weggelassen wird.

Psychische Abhängigkeit: Die psychische Abhängigkeit wird häufig als Ausdruck des Kontrollverlusts, des zwanghaften Konsums und eines starken Cravings beschrieben. Psychische Abhängigkeit bezeichnet das Abhängigkeitssyndrom außerhalb der Dimensionen, die mit dem körperlichen Abhängigkeitssyndrom verbunden sind (Entzugssymptomatik, Toleranzentwicklung).

Psychische Abhängigkeit geht einher mit der individuellen funktionellen Bedeutung des Konsums: Aversive Zustände werden mithilfe des Drogenkonsums überwunden, ein Verzicht auf die Substanz fällt schwer, da antizipierte Wirkeffekte ausbleiben bzw. aversive Effekte erwartet werden. Psychische Abhängigkeit entsteht im Gegensatz zur körperlichen Abhängigkeit vor allem bei einer intendierten Drogeneinnahme, weniger bei einer passiven Verabreichung von Medikamenten.

Sucht: Im allgemeinsprachlichen Gebrauch Synonym für Abhängigkeit mit der Konsequenz der periodischen oder chronischen Intoxikation. Keine Begrifflichkeit im Sinne von ICD-10, ICD-11, DSM-IV und DSM-5. Von der Verwendung des Begriffs wird wegen der mit ihm verbundenen diskriminierenden Assoziationen abgeraten [153].

Opioidabhängigkeit: Die Schwierigkeiten einer differenzierten Abgrenzung einer überwiegend körperlichen $\mathrm{Ab}$ hängigkeit von einer missbräuchlichen Abhängigkeit im Sinne einer Suchterkrankung werden in einem aktuellen Vorschlag von Ballantyne et al. [10] aufgegriffen. Eine Opioidabhängigkeit („opioid dependence“) wird von einer Substanzgebrauchsstörung („opioid use disorder") abgegrenzt. Die Opioidabhängigkeit zeigt sich in Schwierigkeiten einer Dosisreduktion bei langjährig mit hoch dosierten Opioiden behandelten Patienten mit negativer Nutzen/ Risiko-Einschätzung. Für diese refraktäre Opioidabhängigkeit werden folgende diagnostische Kriterien vorgeschlagen: 1. Fehlen von "craving" und Konsumzwang, 2. erhaltene Compliance bezüglich Einnahme, 3. Entzugssymptome, Hyperalgesie, Hyperkatefia, Überempfindlichkeit gegenüber emotionalem Stress, Dysphorie, 4. Schwierigkeiten bei der Dosisreduktion, 5. stressähnliche Symptome, 6. Störung des Belohnungssystems und sozialer Rückzug.

\subsection{Diagnose eines schädlichen Gebrauchs (ICD-10) bzw. Abhängigkeit von Opioiden (ICD-10) bzw. einer "opioid use disorder" (DSM-5)}

Neu 2019. Konsensbasierte Empfehlung: Die Diagnose eines schädlichen Gebrauchs (ICD-10) bzw. einer Abhängigkeit von Opioiden (ICD-10) bzw. einer „opioid use disorder“ des DSM-5 soll nicht bei Patienten gestellt werden, die

- Opioide aus medizinischer Indikation erhalten und

- bei denen neben Toleranzentwicklung und Entzugssymptomen sowie einer mit dem behandelnden Arzt erfolgten Nutzen-SchadenAbwägung von therapeutischen Wirkungen und Nebenwirkungen keine weiteren Merkmale des Missbrauchs und der Abhängigkeit festgestellt werden können. Starker Konsens (97\%; 97\%)

Kommentar: Die diagnostischen Kriterien des ICD-10 und ICD-11 für einen schädlichen Gebrauch bzw. Abhängigkeit von Opioiden (F11.1 und F11.2) bzw. einer „substance use disorder" des DSM-5 wurden nicht für Patienten entwickelt, welche Opioide aus medizinischer Indikation erhalten [25]. Die Kriterien des Substanzkonsums trotz negativer körperlicher, psychischer oder sozialer Konsequenzen (schädlicher Gebrauch) sind nur eingeschränkt anwendbar, da einige Patienten bereit sind, Nebenwirkungen der Opioidmedikation mit negativen körperlichen (z. B. Impotenz, Obstipation), psychischen (z. B. Verschlechterung einer Depression, Müdigkeit, Merk- und Konzentrationsstörungen) oder sozialen Konsequenzen (z. B. Reduktion von Freizeitaktivitäten, Veränderungen im beruflichen Umfeld) wegen der Schmerzlinderung in Kauf zu nehmen. Die Kriterien der Toleranz, der körperlichen Abhängigkeit und des Substanzgebrauchs mit dem Ziel, Entzugssyndrome zu mildern, sind ebenfalls nicht anwendbar, da diese Phänomene auch bei einer Therapie mit Opioiden ohne weitere Hinweise für eine missbräuchliche oder süchtige Verwendung auftreten können.

Folgende Hinweise können auf einen Fehlgebrauch, Missbrauch und Abhängigkeit von aus medizinischer Indikation verschriebenen Opioiden hinweisen [97, 138]:

\section{Nicht-spezifische Hinweise für Fehlgebrauch}

- Einnahme trotz geringer bis fehlender Wirksamkeit

- Ein Fehlgebrauch, der trotz Aufklärung, Absprache und engerer ärztlicher Anbindung anhält, kann als Hinweis auf einen schädlichen Gebrauch/Abhängigkeit gewertet werden.

- Wechselnde Schmerzlokalisationen, multilokuläre Ausbreitung (Generalisierung) der Schmerzen, Transformation des Primärschmerzes unter laufender Therapie

- Opioidinduzierte Hyperalgesie (Tendenz zur Schmerzausbreitung, Erhöhung der Schmerzempfindlichkeit und Opioidresistenz)

\section{Nicht-spezifische Hinweise für Missbrauch/Abhängigkeit}

- Hoher Ruheschmerz sowie Diskrepanz zwischen Schmerzangabe und Verhalten

- Fordern eines bestimmten Opioids, speziell von kurz wirksamen und/ oder schnell anflutenden Opioiden

- Opioideinnahme überwiegend zur Symptomlinderung (Disstress, Unruhe, Angst, Depression, Schlafstörung)

- Nicht abgesprochene Dosiserhöhungen

- Drängen auf Dosiserhöhung ohne Verbesserung der Symptome/ 
Funktion oder trotz Zunahme der Nebenwirkungen

- Wiederholte Unzuverlässigkeiten (Unpünktlichkeit, Nichterscheinen) oder mangelnde Compliance

- Verschwiegene Einnahme von Substanzen mit Suchtpotenzial (Diskrepanzen beim Drug-Monitoring)

- Drängen auf Verschreibung weiterer psychotroper Substanzen

- Veränderung der verabredeten Einnahmeintervalle, eigenständige Anpassung nach Bedarf

- Abwehr von Therapieänderungen

- Wesensveränderungen unter der Therapie (z. B. Impulskontrollstörungen) und andere neue psychiatrische Symptome

- Missbrauch anderer Substanzen zu psychotropen Zwecken

- Zunahme von Depressivität, Angststörung, Albträumen unter der Therapie

\section{Spezifischere Hinweise auf Abhängigkeit}

- Anhaltender Widerstand gegen Änderungen der Medikation trotz

- Wirkungslosigkeit und/oder Symptomen einer ärztlich unerwünschten psychotropen Wirkung (Euphorie, Sedierung, Angstlinderung)

- psychotroper (zumeist dosisabhängiger) Nebenwirkungen (Müdigkeit, Antriebslosigkeit, Konzentrationsstörungen)

- Injektion oraler/transdermaler Verabreichungsformen

- Intravenöse und orale Anwendung transdermaler Systeme

- Rezeptfälschungen

- Stehlen/Borgen von Opioiden

- Nicht-plausibles Horten der Medikamente

- Verheimlichter/abgestrittener Bezug durch andere Ärzte

- Verschwiegener Konsum von weiteren psychotropen Substanzen einschließlich anderer Opioide

- Häufiger Verlust von Rezepten

- Fordern eines parenteralen Verabreichungswegs

- Handel von Opioiden mit Dritten

- Kontrollverlust (z.B. wiederholte Episoden von Dosiserhöhungen oder zunehmend bedarfsgesteuerte Einnahme trotz klarer Absprache/ Warnung, deutliche unmittelbare negative Folgen der Medikamenteneinnahme im privaten und sozialen Umfeld)

- Zwanghafter Gebrauch

\subsection{Einschätzung der Kriterien missbräuchlichen/abhängigen Konsums}

Neu 2019. Konsensbasierte Empfehlung: Zur Einschätzung der genannten Kriterien werden Befunde aus der körperlichen Untersuchung, der Beobachtung des Patientenverhaltens, Angaben aus der Anamnese bzw. Fremdanamnese (Angehörigel Bezugspersonen) und der Therapieverlauf berücksichtigt. Eine interdisziplinäre Beurteilung (z.B. ärztlich, psychologisch, physiotherapeutisch und pflegerisch) kann dazu beitragen, die diagnostische Einordnung zu sichern. Offene Empfehlung. Starker Konsens (100\%; $100 \%)$

\subsection{Bestimmungsgemäßer, nicht-bestimmungsgemäßer und abhängiger Gebrauch}

Neu 2019. Konsensbasierte Feststellung: Es besteht ein fließender Übergang zwischen dem bestimmungsgemäßen Gebrauch, nicht-bestimmungsgemäßen Gebrauch/Konsum („misuse“), schädlichen/ missbräuchlichen („abuse") Gebrauch und abhängigen („süchtigen“) Gebrauch von aus medizinischen Gründen verordneten Opioiden. Starker Konsens (97\%; 97\%)

Kommentar: Beispiele

- Bestimmungsgemäßer Gebrauch: Verordnung und Einnahme von Opioiden bei in LONTS genannten Indikationen (z. B. Postzosterneuralgie)

- Nicht-bestimmungsgemäßer Gebrauch durch Arzt: Verordnung von Opioiden bei in LONTS genannten Kontraindikationen (z. B. primäre Kopfschmerzen)

- Nicht-bestimmungsgemäßer Gebrauch durch Arzt und/oder Patient: Einnahme von bestimmungsgemäß verordneten Opioiden trotz fehlender Wirksamkeit bei in LONTS genannten Indikationen
- Missbräuchliche Verwendung: Einnahme von Opioiden durch Patienten zu psychotropen Zwecken (z. B. Beruhigung) bei in LONTS genannten Indikationen

- Abhängigkeit: Auskochen eines Fentanylpflasters zur intravenösen Injektion zu Rauschzwecken bei in LONTS genannten Indikationen (- Abb. 3)

54.4 Maßnahmen bei Fehlgebrauch oder schädlichem Gebrauch rezeptierter opioidhaltiger Analgetika

Bestätigt 2019. Konsensbasierte Empfehlung: Bei Fehlgebrauch oder schädlichem Gebrauch opioidhaltiger Analgetika sollen Maßnahmen eingeleitet werden, welche die Therapietreue wiederherstellen. Falls diese Maßnahmen nicht erfolgreich sind, soll eine Mitbeurteilung/Mitbehandlung durch Suchtspezialisten eingeleitet werden. Starke Empfehlung. Starker Konsens

Kommentar: Maßnahmen bei Hinweisen für Fehlgebrauch oder Missbrauch (schädlichem Gebrauch)

- Urin- oder, falls indiziert, Haartests oder Blutspiegel als Bedingung für weitere Verschreibungen etablieren

- Erörterung von Verhaltensweisen, welche die Adhärenz beeinträchtigen, und der daraus folgenden Anpassungen des Behandlungsvertrags mit dem Patienten und Personen seines Umfelds

- Einnahme oder Applikation der Medikation unter Aufsicht einer Vertrauensperson

- Zur Gewährleistung der Adhärenz weitere Unterstützung aus dem Umfeld des Patienten suchen

- Reduktionsversuch/Entzug im Rahmen einer multimodalen schmerztherapeutischen Behandlung. Mitbeurteilung/Mitbehandlung des Patienten durch einen Suchtspezialisten. Beendigung der Langzeitanwendung durch qualifizierten Entzug (OPS 9-647)

Erläuterung: Suchtspezialisten: Ärzte mit Qualifikation in suchtmedizinischer Grundversorgung, Ärzte für Psychiatrie und Psychotherapie, Psychologen und Sozialarbeiter an Suchtberatungsstellen 


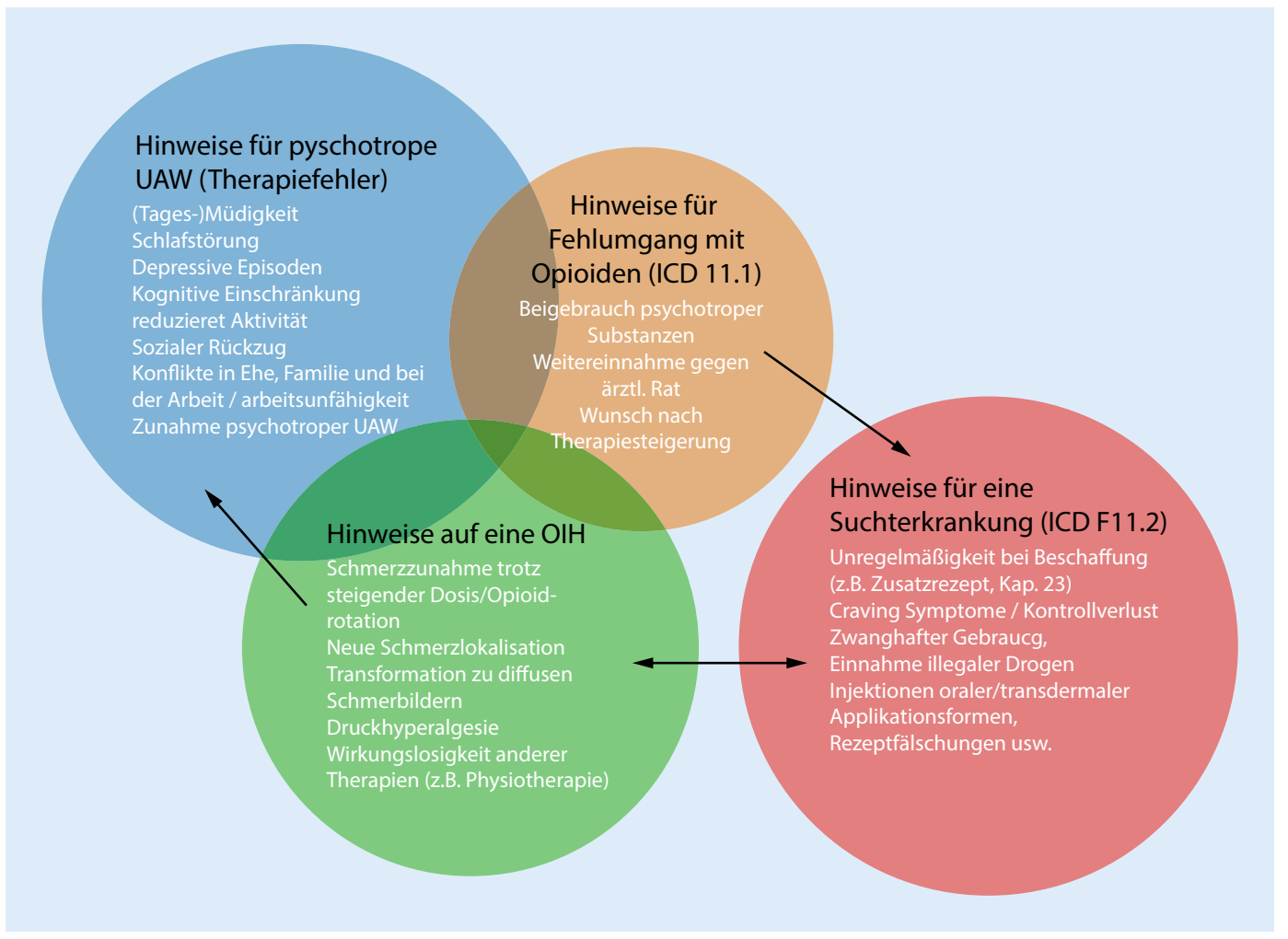

Abb. $3<$ Leitsymptome einer Therapiefehlentwicklung bei Langzeittherapie mit Opioiden. OlH Opiodinduzierte Hyperalgesie. (Mit freundlicherGenehmigung aus [97], $2016 \odot$ C. Maier)

\section{Therapie des schädlichen/ abhängigen Gebrauchs von aus medizinischer Indikation verordneten Opioiden}

Neu 2019. Konsensbasierte Empfehlung: Besteht eine Opioidabhängigkeit, die als unerwünschte Nebenwirkung schmerztherapeutisch verschriebener und erworbener Opioide auftritt, und werden die Opioide missbraucht, im Sinne einer beabsichtigten, ständig oder sporadisch übermäßigen Verwendung mit körperlichen/psychischen Folgen, dann soll folgende Hierarchie an Therapieoptionen angeboten werden:

1. Opioiddosisreduktion/-entzug in multimodaler schmerztherapeutischer Einrichtung

2. Qualifizierter Entzug in suchtmedizinischer psychiatrischer Einrichtung

3. In seltenen Fällen (Notwendigkeit einer analgetischen Therapie mit Opioiden bei anhaltendem Abhängigkeitssyndrom mit Missbrauch der Opioide trotz optimierter Schmerz- und suchtmedizinischer Behandlung): Fortführen der Opioidtherapie als Schmerz- therapie mit suchtmedizinischer Begleitung, als Off-label-Behandlung gemäß $₫ 13$ BtMVV

4. In seltenen und ausgewählten Fällen (Abhängigkeitserkrankung mit anhaltendem Opioidmissbrauch und negativen psychosozialen Folgen und nach Versagen zumindest der unter 1-2 genannten angemessenen suchtmedizinischen und schmerztherapeutischen Maßnahmen zur Begrenzung des Gebrauchs): Aufnahme in eine Substitutionsbehandlung gemäß $\$ 5$ BtMVV. Starke Empfehlung. Starker Konsens $(100 \% ; 100 \%)$

Kommentar: Für die Empfehlung wurde die systematische Literatursuche und -aufbereitung der S3-Leitlinie „Medikamentenabhängigkeit" verwendet. Zur Therapie opioidbezogener Störungen, die sich im Rahmen einer medizinisch indizierten Therapie ergeben haben, wurde eine systematische Literaturrecherche durchgeführt. Aus der systematischen Literaturrecherche wurden aus insgesamt 1032 Treffern zu systematischen Übersichtsarbeiten vier die Fragestellung betreffende systematische
Übersichtsarbeiten identifiziert. Zudem wurden 30 Übersichtsarbeiten identifiziert, die sich mit Therapieoptionen bei nichtmedizinischem Gebrauch von verschreibungspflichtigen Opioiden beschäftigten oder bei denen aus den Einschlusskriterien die gebrauchten Opioide nicht eindeutig bezeichnet waren. Für diese Arbeiten wurde eine mögliche Übertragbarkeit diskutiert. Zusätzlich wurden im Updatezeitraum 2016-2018 der Recherche aus insgesamt 942 randomisierten, kontrollierten Studien zwei relevante Studien ermittelt. Diese Studien zeigen starke Limitationen, die bei der Beurteilung berücksichtigt werden müssen [52].

Eine systematische Übersichtsarbeit analysierte 71 Studien (11 RCTs und 56 Beobachtungsstudien) mit multidisziplinärer Schmerztherapie, buprenorphinunterstützter Dosisreduktion und verhaltenstherapeutischen Interventionen. Die Autoren fanden, dass die genannten Maßnahmen möglicherweise effektiv sind, eine LTOT zu beenden oder zu reduzieren und Schmerzen und Beeinträchtigungserleben zu reduzieren (sehr geringe Qualität der Evidenz) [72]. 
Ein Cochrane-Review analysierte 5 RCTs und 278 Patienten mit Akupunktur, Achtsamkeitstraining und kognitiver Verhaltenstherapie bei Patienten mit CNTS, um die Dosis zu reduzieren bzw. die missbräuchliche Verwendung von Opioiden bei CNTS zu beenden. Die Autoren fanden keine Evidenz für die Wirksamkeit und Sicherheit dieser Maßnahmen [63].

Eine systematische Übersichtsarbeit von 5 RCTs mit 607 Patienten mit "pharmaceutical opioid dependence“ fand, dass Methadon und Buprenorphin gleich wirksam in der Erhaltungstherapie waren. Eine Erhaltungstherapie mit Buprenorphin war der Entgiftung oder psychologischen Verfahren überlegen. Die Qualität der Evidenz war gering bis mäßig [108].

Kontrollierte Studien zum Einsatz von cannabisbasierten Arzneimitteln zur Opioidreduktion wurden nicht gefunden.

Die Entscheidung über verschiedene Therapieoptionen soll von der individuellen klinischen Situation abhängig gemacht werden. Im Falle des Fehlgebrauchs des Opioids (Kontraindikation für Opioide, nicht-opioidsensitiver Schmerz) soll keine Opioidtherapie mehr erfolgen. Mögliche Kriterien für einen stationären Entzug sind erfolglose ambulante Entzüge, instabiles soziales Umfeld und schwere körperliche und/oder psychische Komorbiditäten. Eine Reduktion oder ein Absetzen des Opioids in stationären multimodalen schmerztherapeutischen Einrichtungen ist bei ausreichend motivierten Patienten möglich. In seltenen Fällen (Notwendigkeit einer analgetischen Therapie mit Opioiden bei opioidsensitivem Schmerz und anhaltendem Abhängigkeitssyndrom mit Missbrauch der Opioide trotz optimierter Schmerz- und suchtmedizinischer Behandlung) kann eine Fortführung der Opioidtherapie als Schmerztherapie mit suchtmedizinischer Begleitung als Offlabel-Behandlung gemäß $₫ 13$ BtMVV durchgeführt werden. Bei dieser Konstellation kann auch der Einsatz von Buprenorphin „off label“ erwogen werden. In seltenen und ausgewählten Fällen (Abhängigkeitserkrankung mit anhaltendem Opioidmissbrauch und negativen psychosozialen Folgen und nach Versagen der unter 1-2 genannten suchtmedizinischen und schmerztherapeutischen Maßnahmen zur Begrenzung des Gebrauchs) kann eine Aufnahme in eine Substitutionsbehandlung gemäß $\S 5$ BtMVV erfolgen.

Voraussetzungen für die Substitutionsbehandlung gemäß $₫ 5$ BtMVV von Patientinnen und Patienten mit einer Opioidabhängigkeit im Rahmen einer Schmerzbehandlung sind: Eine Substitutionsbehandlung kann zulässig sein beim Vorliegen einer Opioidabhängigkeit, die als unerwünschte Nebenwirkung rechtmäßig verschriebener und erworbener Opioide auftritt, wenn ein Missbrauch des Opioids vorliegt. In Anlehnung an die Richtlinie 2001/83/EG des Europäischen Parlaments und Rates wurde definiert: „Ein Missbrauch ist ... zu verstehen als die beabsichtigte, ständige oder sporadische übermäßige Verwendung von als Arzneimitteln zugelassenen Opioiden, die iatrogen verschrieben wurden, mit körperlichen und psychischen Schäden als Folge“. Somit dürfen nicht nur die Abhängigkeitskriterien der Toleranz und Entzugssymptomatik vorliegen, sondern es müssen explizit auch weitere Abhängigkeitskriterien zutreffen, wie körperliche und psychische Folgeschäden.

Voraussetzungen für das Erwägen einer Substitutionsbehandlung nach $\$ 5$ BtMVV (alle Kriterien müssen zutreffen) [18]:

1. Es liegt eine Opioidabhängigkeit nach ICD-10 vor. Konkret müssen neben den körperlichen Abhängigkeitskriterien Toleranz und Entzugssymptomen noch weitere Kriterien erfüllt sein. Es liegt ein „Missbrauch“ des Opioidschmerzmittels gemäß der o. a. Definition vor.

2. Alle alternativen schmerzmedizinischen Behandlungsmöglichkeiten sind nachweislich ausgeschöpft.

3. Der Patient/die Patientin ist im schmerztherapeutischen Setting nicht mehr adäquat führbar.

4. Neben den schmerzmedizinischen sollten auch geeignete suchtmedizinische Behandlungsangebote ausgeschöpft sein (vgl. Empfehlungen).

5. Vollendetes 18. Lebensjahr.
6. Ärztliche Stellungnahme der/des vorbehandelnden Ärztin/Arztes mit schmerzmedizinischer Qualifikation, in der das Vorliegen der o. a. Kriterien dargestellt wird und um Prüfung der Durchführung einer Substitutionsbehandlung aus suchtmedizinischer/ psychiatrischer Sicht ersucht wird

Sollte schmerz- und suchttherapeutisch eine Substitutionsbehandlung unter $\mathrm{Zu}$ grundelegung und Dokumentation der o. a. Voraussetzungen befürwortet werden, ist zu berücksichtigen [18]:

a) Die Rahmenbedingungen einer Substitutionsbehandlung gemäß BtMVV und Richtlinien der Bundesärztekammer gelten auch für diese Patientengruppe uneingeschränkt.

b) Die Einnahme des Substitutionsmittels muss zunächst täglich unter Sicht des Fachpersonals in der Substitutionsambulanz/-praxis erfolgen. Hierüber muss der Patient aufgeklärt werden. Das schmerztherapeutische Opioid muss auf ein für eine Substitutionstherapie zugelassenes Opioid umgestellt werden. Als Substitutionsmittel darf nach $₫ 5$ BtMVV der substituierende Arzt nur verschreiben: 1. ein zur Substitution zugelassenes Arzneimittel, das nicht den Stoff Diamorphin enthält, 2. eine Zubereitung von Levomethadon, von Methadon oder von Buprenorphin.

c) Die Einstellung auf die erforderliche Dosis des jeweiligen Substituts muss mit besonderer Sorgfalt erfolgen. Einstiegsdosis und Dosisfindung sind so zu wählen, dass eine Überdosierung vermieden wird. In schwierigen Einzelfällen sollte die Dosisfindung stationär erfolgen. Interagierende Komedikationen sind zu berücksichtigen. Ein die Substitution gefährdender Gebrauch weiterer psychotroper Stoffe einschließlich Alkohol muss bei Einleitung sowie während der Substitution hinsichtlich möglicher Risiken kontrolliert, berücksichtigt und gegebenenfalls begleitend behandelt werden.

d) Erst wenn die Substitution stabil und ohne Beikonsum von nicht ärztlich verordneter Medikation/ psychotroper Stoffe verläuft, kann 
der Patient/die Patientin ein Takehome-Rezept seiner Medikation über maximal 7 Tage, in begründeten Einzelfällen auch über einen längeren Zeitraum erhalten. In der Regel muss einmal pro Woche eine Einnahme des Opioidsubstitutionsmittels unter Sicht des Fachpersonals in der Substitutionsambulanz erfolgen.

e) Vor einer Entscheidung für eine Substitutionsbehandlung ist der Patient/die Patientin auch darüber aufzuklären, dass es sich bei einer Substitutionstherapie nach den Erfahrungen der Substitutionstherapie primär Opioidabhängiger um eine lang andauernde, in manchen Fällen auch lebenslange Behandlungsform handelt.

In der von der Deutschen Gesellschaft für Psychiatrie, Psychotherapie, Psychosomatik und Nervenheilkunde koordinierten S3-Leitlinie „Medikamentenbezogene Störungen“, welche im Laufe des Jahres 2020 fertiggestellt werden wird [52], wird es weitere Hinweise zum Vorgehen einer Substitutionsbehandlung bei Schmerzpatienten geben.

\section{Konsensbasierte Empfehlung: medikamentöse Unterstützung einer Reduktion oder eines vollständigen Absetzens von Opioiden}

Neu 2019. Konsensbasierte Feststellung: Ein Opioidentzug kann ohne und mit medikamentöser Unterstützung (z.B. trizyklische Antidepressiva, Gabapentinoide, Clonidin) durchgeführt werden. Offene Empfehlung. Starker Konsens (100\%; $100 \%)$

Kommentar: Es wurden keine kontrollierten Studien zur medikamentösen Unterstützung eines Opioidentzugs bei $\mathrm{Pa}$ tienten, die aus medizinischer Indikation Opioide erhielten, gefunden.

$\mathrm{Ob}$ ein abruptes Absetzen der Opioide und eine Gabe von Medikamenten zur Reduktion von Entzugssymptomen (z.B. trizyklische Antidepressiva, Gabapentinoide, Clonidin) oder eine langsame Reduktion der Opioiddosis mit bedarfsweiser Gabe von Medikamenten zur Reduktion von Entzugssymptomen von Patienten besser toleriert wird bzw. zu langfristiger Opioidabstinenz führt, ist nicht durch kontrollierte Studien untersucht.

Wenn eine medikamentöse Unterstützung des Entzugs durchgeführt wird, sollen bei der Wahl des Medikaments die Art der Entzugssymptome sowie körperliche und psychische Komorbiditäten berücksichtigt werden. Eine Unterstützung des Entzugs durch Medikamente mit Abhängigkeitspotential soll bei $\mathrm{Pa}$ tienten mit multipler Substanzabhängigkeit nur in Ausnahmefällen durchgeführt werden. Von den genannten Substanzen ist nur Doxepin in Deutschland zur Entzugsbehandlung zugelassen.

Ein Opioidentzug kann mit dem missbräuchlich verwendeten Opioid oder mit Morphin (siehe Praxiswerkzeug) durchgeführt werden.

\section{Neu}

- Praxiswerkzeuge: Opioidentzugsbehandlung bei Schmerzpatienten und Patientenvereinbarung über Medikamentenentzug https://www.awmf.org/leitlinien/ detail/ll/145-003.html

\section{Prävention: Absetzen von Opioiden im Rahmen einer Akutschmerztherapie}

Neu 2019. Klinischer Konsensuspunkt: Die Indikation für die Fortführung einer Akutschmerztherapie mit Opioiden wegen operativer oder interventioneller Eingriffe soll vor und nach Entlassung aus stationärer Behandlung überprüft und bei fehlender Indikation für eine Langzeittherapie die Opioidmedikation nach stationärer Entlassung zügig beendet werden. Starke Empfehlung. Starker Konsens (97\%; 97\%)

Kommentar: Ein Teil der Opioidepidemie in den USA wird auf die Fortführung einer im Krankenhaus zur Akutschmerztherapie begonnenen Behandlung mit Opioiden zurückgeführt [151]. Aus Deutschland liegen keine Daten vor, wie viel Prozent der Langzeitanwendungen von Opioiden im Rahmen einer stationären Akutschmerztherapie begonnen wurden. Eine frühzeitige schmerztherapeutische Mitbehandlung sollte bei anhaltendem Opioidbedarf nach Ablauf der üblichen Zeit von postoperativen bzw. -interventionellen Schmerzen im Einzelfall auch schon vor der Entlassung eingeleitet werden.

\section{Diskussion}

In Anbetracht der unterschiedlichen Interessen der an der Leitlinie beteiligten Personen (Betroffene und Behandler, Ärzte und Psychologen, Allgemein- und Fachärzte, niedergelassene Ärzte und Kliniker, Pflege und Apotheker) ist der starke Konsens, der bei fast allen Empfehlungen erreicht wurde, hervorzuheben.

Gegenüber der ersten Aktualisierung erfolgten folgende inhaltliche Änderungen:

Präambel: Es wurden evidenzbasierte Feststellungen zu Definitionen der Langzeitanwendung von Opioiden, zur Häufigkeit der missbräuchlichen/ abhängigen Verwendung von aus medizinischer Indikation verschriebenen opioidhaltigen Analgetika und zu einer möglichen Opioidepidemie in Deutschland ergänzt.

Evidenz- und konsensbasierte Indikationen: Es wurde - basierend auf den durchgeführten Metaanalysen - eine Differenzierung zwischen einem 4- bis 12wöchigen, 13- bis 26-wöchigen und >26wöchigen Einsatz durchgeführt. Bei fehlender Evidenz für einen länger als 12wöchigen Einsatz wurde die weitere Verwendung von opioidhaltigen Analgetika auf Therapieresponder beschränkt.

Neu aufgenommen wurden evidenzbasierte Empfehlungen zum Restlesslegs-Syndrom und Parkinson-Syndrom und konsensbasierte Empfehlungen zu traumatischer Trigeminusneuropathie und chronischem Unterbauchschmerz der Frau bei ausgeprägten Verwachsungen und/oder multilokulärer Endometriose.

Neu aufgenommen wurde eine Empfehlung zum Stellenwert einer intrathekalen Opioidtherapie mit Praxiswerkzeug.

Praxis der Opioidtherapie: Die potenziellen Indikationen für Opioide wurden durch folgende konsensbasierte Empfehlungen weiter eingeschränkt:

a) Vor Einleitung einer Therapie mit

Opioiden sollen die nicht-me-

dikamentösen Therapieoptionen 
optimiert und medikamentöse Alternativen erwogen werden.

b) Die kurz-, mittel- und langfristige Anwendung opioidhaltiger Analgetika soll auf Patienten mit einem nach ärztlich/psychologisch/ physiotherapeutischer Einschätzung relevanten somatischen Anteil in der Schmerzentstehung und Aufrechterhaltung und unzureichendem Ansprechen auf nicht-medikamentöse Therapien beschränkt werden.

c) Die Anwendung opioidhaltiger Analgetika bei Patienten mit Arthroseschmerzen soll auf folgende klinische Konstellationen beschränkt werden: Gelenkersatz nicht möglich oder von Patienten nicht gewünscht; Versagen nicht-medikamentöser Therapien; Wirkungslosigkeit bzw. Kontraindikationen anderer Analgetika

Neue Empfehlungen: Aufklärung über folgende potenzielle Nebenwirkungen:

Erhöhtes Sturzrisiko; sexuelle Funktionsstörungen; endokrine Funktionsstörungen; schlafbezogene Atmungsstörungen.

Stark negative Empfehlung zu einer Kombinationstherapie von Opioiden mit Tranquilizern

Überprüfung möglicher Arzneimittelwechselwirkungen mit einem Praxiswerkzeug

Aushändigung eines bundeseinheitlichen Medikationsplans

Aktualisierung des Praxiswerkzeugs zur Therapie der opioidinduzierten Obstipation und Definition des Stellenwerts der peripheren Opioidantagonisten

Empfehlungen bei Patienten mit Atemstörungen

In enger Absprache mit der parallel entwickelten S3-Leitlinie „Medikamentenabhängigkeit", an der die beiden Koordinatoren dieser Leitlinie für die Deutsche Schmerzgesellschaft teilnahmen, wurden Empfehlungen zur Diagnostik und Therapie der missbräuchlichen/ abhängigen Verwendung von Opioiden, die aus medizinischer Indikation verschriebenen wurden, erstellt.

Folgende Forschungsdesiderata bestehen nach Einschätzung der Steuergruppe unverändert zur ersten Aktualisierung:
- Zur Frage der Langzeitrisiken und Fehlversorgung sind Analysen von Routinedaten der Krankenkassen notwendig.

- Die klinische Prüfung der Frage nach Nutzen und Nebenwirkungen beim Vergleich „Opioid nach festem Zeitschema (zeitkontingent)“ vs. „Opioid nach Bedarf“ im Rahmen einer ausreichend gepowerten Studie mit geeignetem Design ist sinnvoll.

\section{Korrespondenzadresse}

\section{Prof. Dr. med. Winfried Häuser}

Medizinisches Versorgungszentrum für Schmerzmedizin und seelische Gesundheit, Saarbrücken St. Johann Großherzog-Friedrich-Straße 44-46, 66111 Saarbrücken, Deutschland whaeuser@klinikum-saarbruecken.de

Koautoren für die Mitglieder der Konsensusgrup pe der 2. Aktualisierung der S3-Leitlinie LONTS. Karl-Jürgen Bär; Christoph Baerwald; Matthias Beintker; Jens Büntzel; Corinna Elling-Audersch; Stephan Freys; Irmela Gnass; Ursula Havemann-Reinecke; Kirstin Hupfer; Ulrich Kellner; Claas Lahmann; Martin Marziniak; Gerhard Müller; Holger Petri; Achim Rody; Michael Schäfer; Dietmar Schöffel; Volker Thieme; Volker Tronnier; Dirk Wolter; Dan Ziegler

\section{Einhaltung ethischer Richtlinien}

Interessenkonflikt. Die Interessenkonflikte aller Autoren und Koautoren sind im Leitlinienreport (https:// doi.org/10.1007/s00482-020-00471-z) in dieser Ausgabe aufgeführt.

Für diesen Beitrag wurden von den Autoren keine Studien an Menschen oder Tieren durchgeführt. Für die aufgeführten Studien gelten die jeweils dort angegebenen ethischen Richtlinien.

\section{Literatur}

1. Abbasi $A B$, Salisbury-Afshar $E$, Jovanov $D$, Berberet $C$, Arunkumar P, Aks SE, Layden JE, Pho MT (2019) Health care utilization of opioid overdose decedents with no opioid analgesic prescription history. JUrban Health 96:38-48

2. Allan L, Richarz U, Simpson K, Slappendel R (2005) Transdermal fentanyl versus sustained release oral morphine in strong-opioid naïve patients with chronic low back pain. Spine (Phila Pa 1976) 3:2484-2490

3. AminiLari M, Manjoo $P$, Craigie $S$, Couban $R$, Wang L, Busse JW (2019) Hormone replacement therapy and opioid tapering for opioid-induced hypogonadism among patients with chronic noncancer pain: a systematic review. Pain Med 20:301-313
4. American Psychiatric Association (2013) Diagnostic and statistical manual of mental disorders, 5. Aufl. American Psychiatric Publishing, Washington

5. Andresen V, Enck P, Frieling T (2013) S2kLeitlinie Chronische Obstipation: Definition, Pathophysiologie, Diagnostik und Therapie. ZGastroenterol 51:651-672

6. Atzendorf J, Rauschert C, Seitz NN, Lochbühler K, Kraus $L$ (2019) The use of alcohol, tobacco, illegal drugs and medicines-an estimate of consumption and substance-related disorders in Germany. Dtsch Arztebl Int 116:577-584

7. Aydin D (2018) Konsumstörungen im Zusammenhang mit opioidhaltigen und nicht opioidhaltigen Schmerzmitteln - Prävalenz in der Bevölkerung und prädiktive Effekte (Bachelorarbeit). FernUniversität in Hagen, Hagen

8. Bader S, Weber M, Becker G (2012) Is the pharmacological treatment of constipation in palliative care evidence based?: A systematic literature review. Schmerz 26:568-586

9. Baker DW (2017) History of the Joint Commission's pain standards: lessons for today's prescription opioid epidemic. JAMA 317:1117-1118

10. Ballantyne JC, Sullivan MD, Koob GF (2019) Refractory dependence on opioid analgesics. Pain 160:2655-2660

11. Barrera-Chacon JM, Mendez-Suarez JL, JaureguiAbrisqueta ML, Palazon R, Barbara-Bataller E, Garcia-Obrero I (2011) Oxycodone improves pain control and quality of life in anticonvulsantpretreated spinal cord-injured patients with neuropathic pain. Spinal Cord 49:36-42

12. Bialas $P$, Maier C, Klose $P$, Häuser W (2020) Efficacy and harms of long-term opioid therapy in chronic non-cancer pain: Systematic review and metaanalysis of open-label extension trials with a study duration $\geq 26$ weeks. Eur J Pain 24:265-278

13. Biondi DM, Xiang J, Etropolski M, Moskovitz B (2015) Tolerability and efficacy of tapentadol extended release in elderly patients $\geq 75$ years of age with chronic osteoarthritis knee or low back pain. JOpioid Manag 11:393-403

14. Bilal M, Chatila A, Siddiqui MT, Al-Hanayneh M, Shah AR, Desai M, Wadhwa V, ParupudiS, Casey BW, Krishnan K, Hernandez-Barco YG (2019) Rising prevalence of Opioid use disorder and predictors for opioid use disorder among hospitalized patients with chronic pancreatitis. Pancreas 48:1386-1392

15. Böger RH, Schmidt G (2019) Analgetika. In: Schwabe U, Paffrath D, Ludwig WD, Klauber J (Hrsg) Arzneiverordnungsreport 2019. Springer, Heidelberg, Berlin, New York, S389-406

16. Bone C, Gelberg L, Vahidi M, Leake B, YacendaMurphy J, Andersen RM (2016) Under-reporting of risky drug use among primary care patients in federally qualified health centers. J Addict Med 10:387-394

17. Brunnmüller $U$, Zeitker $H$, Alten $R$, Gromnica-Ilhle $E$ (2004) Wirksame Schmerztherapie mit Tidlin/ Naloxon retard bei Patienten mit rheumatoider Arthritis. Akt Rheumatol 29:35-39

18. Bundesärztekammer (2017) Richtlinien zur Substitutionsgestützten Behandlung der Bundesärztekammer. https://www.bundesaerztekammer.de/ fileadmin/user_upload/downloads/pdf-Ordner/ RL/Substitution.pdf.Zugegriffen:3. Okt. 2019

19. Bundesärztekammer (BÄK), Kassenärztliche Bundesvereinigung (KBV), Arbeitsgemeinschaft der Wissenschaftlichen Medizinischen Fachgesellschaften (AWMF) (2011) Nationale Versorgungsleitlinie Neuropathie bei Diabetes 
im Erwachsenenalter. http://www.diabetes. versorgungsleitlinien.de. Zugegriffen: 1. Febr. 2014

20. Burr NE, Smith C, West R, Hull MA, Subramanian V (2018) Increasing prescription of opiates and mortality in patients with inflammatory bowel diseases in England. Clin Gastroenterol Hepatol 16:534-541

21. Busse JW, Craigie S, JuurlinkDN, Bucley DN, Wang L, Couban RJ, Agoritsas T, AkI EA, Carrasco-Labra A, Cooper L, Cull C, da Costa BR, Frank JW, Grant G, lorio A, Persaud N, Stern S, Tugwell P, Vandvik PO, Guyatt GH (2017) Guideline for opioid therapy and chronicnoncancer pain. Cmaj 189:E659-E666

22. Buth S, Holzbach R, Rosenkranz M, Verthein U (2017) Der Gebrauch von Medikamenten mit Abhängigkeitspotenzial in Deutschland: Eine prospektive Analyse kassenärztlicher Verschreibungen der Jahre 2006 bis 2010. Bundesgesundheitsblatt Gesundheitsforschung Gesundheitsschutz 60(8):865-872

23. Buth S, Holzbach R, Martens MS, NeumannRunde E, Meiners O, Verthein U (2019) Problematic medication with benzodiazepines, "Z-drugs", and opioid analgesics-An analysis of national health insurance prescription data from 2006-2016. Dtsch Arztebl Int 116:607-614

24. Campbell JN (1996) Pain as a 5. Vital sign. APS 1995 presidential address. Pain Forum 1:85-88

25. Campbell G, Bruno R, Lintzeris N, Cohen $M$, Nielsen S, Hall W, Larance B, Mattick RP, Blyth F, Farrell M, Degenhardt L (2016) Defining problematic pharmaceutical opioid use among people prescribed opioids for chronic noncancer pain: do different measures identify the same patients? Pain 157:1489-1498

26. Casser HR, Arnold B, Brinkschmidt T, Gralow I, Irnich D, Klimczyk K, Nagel B, Pfingsten M, Sabatowski R, Schiltenwolf M, Sittl R, Söllner W (2013) Multidisciplinary assessment for multimodal pain therapy. Indications and range of performance. Schmerz 27:363-370

27. Centers for Disease Control and Prevention (CDC) (2015) Number and age-adjusted rates of drugpoisoning deaths involving opioid analgesics and heroin: United States, 2000-2014. National Vital Statistics System, Mortality File. http://wonder. cdc.gov/.Zugegriffen:6.Jan. 2019

28. Centers for Disease Control and Prevention (2018) 2018 annual surveillance report of drug-related risks and outcomes-United States. Surveillance Special Report. Centers for Disease Control and Prevention, U.S. Department of Health and Human Services. https://www.cdc.gov/drugoverdose/ pdf/pubs/2018-cdc-drug-surveillance-report. pdf (Erstellt: 31. Aug. 2018). Zugegriffen: 6. Jan. 2019

29. Chaparro LE, Furlan AD, Deshpande A et al (2013) Opioids compared to placebo or other treatments for chronic low-back pain. Cochrane Database Syst Rev 8:CD4959

30. Charité Universitätsmedizin (2019) www. embryotox.de.Zugegriffen: 1. Aug. 2019

31. Chenot JF, Greitemann B, Kladny B, Petzke F, Pfingsten M, Schorr SG (2017) Non-specific low back pain. Dtsch Arztebl Int 114:883-890

32. Chou R, Turner JA, Devine EB, Hansen RN, Sullivan SD, Blazina I, Dana T, Bougatsos C, Deyo RA (2015) The effectiveness and risks of long-term opioid therapy for chronic pain: a systematic review for a National Institutes of Health Pathways to Prevention Workshop. Ann Intern Med 162:276-286
33. Cicero TJ, Ellis MS (2017) The prescription opioid epidemic: a review of qualitative studies on the progression from initial use to abuse. Dialogues Clin Neurosci 19:259-269

34. Clegg A, Young JB (2011) Which medications to avoid in people at risk of delirium: a systematic review. Age Ageing 40:23-29

35. Coates MD, Seth N, Clarke K, Abdul-Baki H, Mahoney N, Walter V, Regueiro MD, Ramos-Rivers C Koutroubakis IE, Bielefeldt K, Binion DG (2019) Opioid analgesics do not improve abdominal pain or quality of life in Crohn's disease. Dig Dis Sci. https://doi.org/10.1007/s10620-019-05968-x

36. Coluzzi F, Billeci D, Maggi M, Corona G (2018) Testosterone deficiency in non-cancer opioidtreated patients. JEndocrinol Invest 41:1377-1388

37. da Costa BR, Reichenbach S, Keller N, Nartey L, Wandel S, Jüni P, Trelle S (2017) Effectiveness of non-steroidal anti-inflammatory drugs for the treatment of pain in knee and hip osteoarthritis: a network meta-analysis. Lancet 390:e21-e33

38. Correa D, Farney RJ, Chung F, Prasad A, Lam D, Wong J (2015) Chronic opioid use and central sleep apnea: a review of the prevalence, mechanisms, and perioperative considerations. Anesth Analg 120:1273-1285

39. Dasgupta N, Funk MJ, Proescholdbell S, Hirsch A, Ribisl KM, Marshall S (2016) Cohort study of the impact of high-dose opioid analgesics on overdose mortality. Pain Med 17:85-98

40. Davis MP, Mehta Z (2016) Opioids and chronic pain: Where is the balance? Curr Oncol Rep 18:71

41. Deutscher Bundestag (2019) Keine Opioidkrise wie in den USA. https://www.bundestag.de/presse/ hib/635928-635928. Zugegriffen: 10. Aug. 2019

42. DeWeerdt S (2019) The natural history of an epidemic. Nature 573:S10-S12

43. Deutsche Gesellschaft für Neurologie (2014) Therapie des episodischen und chronischen Kopfschmerzes vom Spannungstyp und anderer chronischer täglicher Kopfschmerzen. https://www. dgn.org/leitlinien/3019-II-56-II-therapie-desepisodischen-und-chronischen-kopfschmerzesvom-spannungstyp.Zugegriffen: 16. Aug. 2019

44. Deutsche Gesellschaft für Neurologie (2017) Cervicale Radikulopathie. https://www.awmf.org/ uploads/tx_szleitlinien/030-082l_S2k_Zervikale_ Radikulopathie_2018-01.pdf. Zugegriffen: 16. Aug. 2019

45. Deutsche Gesellschaft für Neurologie (2018) Lumbale Radikulopathie. https://www.awmf.org/ uploads/tx szleitlinien/030-058I S2k_Lumbale Radikulopathie_2018-04.pdfZugriff.Zugegriffen: 16. Aug. 2019

46. Deutsche Gesellschaft für Neurologie (2018) Diagnose und nicht-interventionelle Therapie neuropathischer Schmerzen. https://www.awmf. org/leitlinien/detail/II/030-114.html. Zugegriffen: 28. Nov. 2019

47. Deutsche Gesellschaft für Neurologie (2018) Therapie der Migräneattacke und Prophylaxe der Migräne. https://www.awmf.org/leitlinien/detail/ II/030-057.html. Zugegriffen: 16. Aug. 2019

48. Deutsche Gesellschaft für Orthopädie und Orthopädische Chirurgie (DGOOC), Berufsverband der Ärzte für Orthopädie (BVO) (2018) Leitlinie Coxarthrose. https://www.awmf.org/uploads/ tx szleitlinien/033-001I_S2k Koxarthrose_201907_1.pdf.Zugegriffen:10.Aug. 2019

49. Deutsche Gesellschaft für Orthopädie und Orthopädische Unfallchirurgie (2018) S2k Leitlinie spezifischer Kreuzschmerz. https://www. awmf.org/uploads/tx_szleitlinien/033-051I_S2k_
Spezifischer Kreuzschmerz 2018-02.pdf. Zugegriffen: 10. Aug. 2019

50. Deutsche Gesellschaft für Orthopädie und Orthopädische Unfallchirurgie (2018) S2k Leitlinie Gonarthrose. https://www.awmf.org/uploads/tx szleitlinien/033-004l_S2k_Gonarthrose_201801_1.pdf $+\& c d=2 \& h l=d e \& c t=c l n k \& g l=d e . ~ Z u g e-$ griffen: 14. Nov. 2019

51. Deutsche Gesellschaft für Psychosomatische Frauenheilkunde und Geburtshilfe (DGPfG) (2014) Leitlinie chronischer Unterbauchschmerz der Frau. http://www.awmf.org/uploads/tx_szleitlinien/ 016-0011_abgelaufen.pdf. Zugegriffen: 1. Febr. 2014

52. Deutsche Gesellschaft für Psychiatrie, Psychotherapie, Nervenheilkunde und Psychosomatische Medizin (2020) Leitlinie Medikamentenabhängigkeit

53. Deutsche Gesellschaft für Rheumatologie (2019) Management der frühen rheumatoiden Arthritis. https://www.awmf.org/leitlinien/detail/II/060002.html.Zugegriffen:31.Okt. 2019

54. Deutschsprachige Medizinische Gesellschaft für Paraplegie (2018) Leitlinie Schmerzen bei Querschnittslähmung. https://www.awmf. org/uploads/tx_szleitlinien/179-006l_S2k Schmerzen_Querschnittlaehmung_2018-08.pdf. Zugegriffen: 1.Juli 2019

55. Deutsche Krebsgesellschaft (2018) Erweiterte S3-Leitlinie Palliativmedizin für Patienten mit einer nicht-heilbaren Krebserkrankung. https:// www.awmf.org/...szleitlinien/128-0010LI_KF S3_Palliativmedizin_2018-12.pdf.Zugegriffen: 7. März 2019

56. Deutsches Institut für Medizinische Information und Dokumentation (2019) OPS 2019. https:// www.dimdi.de/dynamic/de/klassifikationen/ ops/.Zugegriffen:3.Okt. 2019

57. Diasso PDK, Birke H, Nielsen SD, Main KM, Højsted J, Sjøgren P, Kurita GP (2019) The effects of long-term opioid treatment on the immune system in chronic non-cancer pain patients: A systematic review. Eur JPain 24(2):481-496

58. Die Drogenbeauftragte der Bundesregierung (2018) Drogen- und Suchtbericht Juli 2018. https:// www.mdr.de/nachrichten/politik/drogen-undsuchtbericht-100.html.Zugegriffen: 1. Aug. 2019

59. Dowell D, Haegerich TM, Chou R (2016) CDC guideline for prescribing opioids for chronic pain-United States, 2016. JAMA 315:1624-1645

60. Drewes AM, Munkholm P, Simrén M, Breivik $H$, Kongsgaard UE, Hatlebakk JG, Agreus L, Friedrichsen M, Christrup LL (2016) Definition, diagnosis and treatment strategies for opioid-induced bowel dysfunction-Recommendations of the Nordic Working Group. Scand J Pain 11:111-122

61. Dubrall D, Schmid M, Alešik E, Paeschke N, Stingl J, Sachs B (2018) Frequent adverse drug reactions, and medication groups under suspicion. Dtsch Arztebl Int 115:393-400

62. Dworkin RH, Turk DC, Wyrwich KW, Beaton D, Cleeland CS, Farrar JT, Haythornthwaite JA, Jensen MP, Kerns RD, Ader DN, Brandenburg N, Burke LB, Cella D, Chandler J, Cowan P, Dimitrova R, Dionne R, Hertz S, Jadad AR, Katz NP, Kehlet H, Kramer LD, Manning DC, McCormick C, McDermott MP, MCQuay HJ, Patel S, Porter L, Quessy S, Rappaport BA, Rauschkolb C, Revicki DA, Rothman M, Schmader KE, Stacey BR, Stauffer JW, von Stein T, White RE, Witter J, Zavisic S (2008) Interpreting the clinical importance of treatment outcomes in chronic pain clinical trials: IMMPACT recommendations. J Pain 9:105-121 
63. Eccleston C, Fisher $\mathrm{E}$, Thomas $\mathrm{KH}$, Hearn $\mathrm{L}$, Derry S, Stannard C, Knaggs R, Moore RA (2017) Interventions for the reduction of prescribed opioid use in chronic non-cancer pain. Cochrane Database Syst Rev 11:CD10323

64. Els C, Jackson TD, KunykD, Lappi VG, Sonnenberg B, Hagtvedt R, Sharma S, Kolahdooz F, Straube S (2017) Adverse events associated with mediumand long-term use of opioids for chronic noncancer pain: an overview of Cochrane Reviews. Cochrane Database Syst Rev 10:CD12509

65. Falco FJ, Patel VB, Hayek SM, Deer TR, Geffert S, Zhu J, Onyewu O, Coubarous S, Smith HS, Manchikanti L (2013) Intrathecal infusion systems for long-term management of chronic non-cancer pain: an update of assessment of evidence. Pain Physician 16(2Suppl):SE185-216

66. Flöter T, Brunnmüller U (2002) Tilidine/naloxon retard in long-term administration in chronic pain and multimorbidity. Multicenter study of long-term tolerance and effectiveness in 2 years observation. Fortschr Med Orig 120:29-35

67. Foodand Drug Admininstration (2019)FDA In Brief: FDA requires new warnings for gabapentinoids about risk of respiratory depression. https://www. fda.gov/news-events/fda-brief/fda-brief-fdarequires-new-warnings-gabapentinoids-aboutrisk-respiratory-depression. Zugegriffen: 30. Jan. 2020

68. Foong AL, Grindrod KA, Patel T, Kellar J (2018) Demystifying serotonin syndrome (or serotonin toxicity). Can Fam Physician 64:720-727

69. Ford AC, Brenner DM, Schoenfeld PS (2013) Efficacy of pharmacological therapies for the treatment of opioid-induced constipation: systematic review and meta-analysis. Am J Gastroenterol 108:1566-1574

70. Franchi S, Amodeo G, Gandolla M, Moschetti G, Panerai AE, Sacerdote $P$ (2017) Effect of tapentadol on splenic cytokine production in mice. Anesth Analg 124:986-995

71. Franchi S, Moschetti G, Amodeo G, Sacerdote $P$ (2019) Do all opioid drugs share the same Immunomodulatory properties? A review from animal and humanstudies. Front Immunol 10:2914

72. Frank JW, Lovejoy TI, Becker WC, Morasco BJ, Koenig CJ, Hoffecker L, Dischinger HR, Dobscha SK, KrebsEE(2017) Patientoutcomes in dose reduction or discontinuation of long-term opioid therapy: a systematic review. Ann Intern Med 167:181-191

73. Freedman MD, Schwartz HJ, Roby R et al (1997) Tolerance and efficacy of polyethylene glycol $3350 /$ electrolyte solution versus lactulose in relieving opiate induced constipation: a doubleblinded placebo-controlled trial. J Clin Pharmacol 37:904-907

74. Furlan A, Chaparro LE, Irvin E, Mailis-Gagnon A (2011) A comparison between enriched and nonenriched enrollment randomized withdrawal trials of opioids for chronic noncancer pain. Pain Res Manag 16:337-351

75. Gaither JR, Goulet JL, Becker WC, Crystal S, Edelman JE, Gordon K, Kerns RD, Rimland D, Skandersson M, Justice AC, Fiellin DA (2016) The association between receipt of guidelineconcordant long-term opioid therapy and allcause mortality. J Gen Intern Med 31:492-501

76. Garg RK, Fulton-Kehoe D, Franklin GM (2017) Patterns of Opioid use and risk of opioid overdose death among medicaid patients. Med Care 55:661-668

77. Gomes T, Juurlink DN, Antoniou T, Mamdani MM, Paterson JM, van den Brink W (2017) Gabapentin, opioids, and the risk of opioid-related death: a population-based nested cased control study. PLoSMed 14(10):e1002396

78. Grayston R, Czanner G, Elhadd K, Goebel A, Frank B, Üçeyler N, Malik RA, Alam U (2019) A systematic reviewand meta-analysis of the prevalence of small fiber pathology in fibromyalgia: Implications for a new paradigm in fibromyalgia etiopathogenesis. Semin Arthritis Rheum 48:933-940

79. Hadley G, Derry S, Moore RA, Wiffen PJ (2013) Transdermal fentanyl for cancer pain. Cochrane Database Syst Rev 10:CD10270

80. Han B, Compton WM, Blanco C, Crane E, Lee J, Jones CM (2017) Prescription opioid use, misuse, and use disorders in U.S. Adults: 2015 national survey on drug use and health. Ann Intern Med 167:293-301

81. Harden RN, Kaye AD, Kintanar T, Argoff CE (2013) Evidence-based guidance for the management of postherpetic neuralgia in primary care. Postgrad Med 125(4):191-202

82. Häuser W, Bock F, Engeser P, Hege-Scheuing G, Hüppe M, Lindena G, Maier C, Norda H, Radbruch L, Sabatowski R, SchäferM, SchiltenwolfM, SchulerM, Sorgatz H, Tölle T, Willweber-Strumpf A, Petzke F (2015) Recommendations of the updated LONTS guidelines: Long-term opioid therapy for chronic noncancer pain. Schmerz 29:109-130

83. Häuser W, Schubert T, Scherbaum N, Tölle T (2018) Guideline-recommended vs high-dose long-term opioid therapy for chronic noncancer pain is associated with better health outcomes: data from a representative sample of the German population. Pain 159:85-91

84. Häuser W, Schubert T, Scherbaum N, Tölle T (2018) Long-term opioid therapy of non-cancer pain: Prevalence and predictors of hospitalization in the event of possible misuse. Schmerz 32:419-426

85. Häuser W, Perrot S, Clauw DJ, Fitzcharles MA (2018) Unravelling fibromyalgia-steps toward individualized management. J Pain 19:125-134

86. Health Canada (2019) Health Canada advises Canadians to exercise caution when taking gabapentin or pregabalin with opioids. http:// healthycanadians.gc.ca/recall-alert-rappel-avis/ hc-sc/2019/71003a-eng.php. Zugegriffen:30. Jan. 2020

87. Hopf HC, Philipowich G (2008) Behandlung mit noch nicht zugelassenen Medikamenten: Zwischen Hoffen und Haften. Dtsch Arztebl 105:A552/B-494/C-482

88. Itz CJ, Geurts JW, van Kleef M, Nelemans P (2013) Clinical course of non-specific low back pain: a systematic review of prospective cohort studies set in primary care. Eur J Pain 17:5-15

89. KarroumEG, Golmard JL, Leu-Semenescu S, Arnulf I (2015) Painful restless legs syndrome: a severe, burning form of the disease. Clin JPain 31:459-466

90. Kiesel EK, Hopf YM, Drey M (2018) An anticholinergic burden score for German prescribers: score development. BMC Geriatr 18:239

91. Kopecky EA, Vaughn B, Lagasse S, O'Connor M (2017) Tolerability, safety, and effectiveness of oxycodone DETERx in elderly patients $\geq 65$ years of age with chronic low back pain: a randomized controlled trial. Drugs Aging 34:603-613

92. Kraus L, Seitz NN, Schulte B et al (2019) Estimation of the number of people with opioid addiction in Germany. Dtsch Arztebl Int 116:137-143

93. Krüger R, Meißner W, Zimmer A (2014) Misuse of opioid analgesics. An internet analysis. Schmerz 28:473-482

94. Layer P, Andresen V, PehlC, Allescher H, BischoffSC, Classen M, Enck P, Frieling T, Haag S, Holtmann G, Karaus M, Kathemann S, Keller J, Kuhlbusch-Zick- lam R, Kruis W, Langhorst J, Matthes H, Mönnikes H, Müller-Lissner S, Musial F, Otto B, Rosenberger C, Schemann M, van der Voort I, Dathe K, Preiss JC, Deutschen Gesellschaft für Verdauungs- und Stoffwechselkrankheiten Deutschen Gesellschaft für Neurogastroenterologie und Motilität (2011) Irritable bowel syndrome: German consensus guidelines on definition, pathophysiology and management. ZGastroenterol 49:237-293

95. Lee M, Silverman SM, Hansen $\mathrm{H}$ et al (2011) A comprehensive review of opioid-induced hyperalgesia. Pain Physician 14:145-161

96. Macfarlane GJ, Kronisch C, Dean LE, Atzeni F, Häuser W, FlußE, Choy E, KosekE, Amris K, Branco J, Dincer F, Leino-Arjas P, Longley K, McCarthy GM, Makri S, Perrot S, Sarzi-Puttini P, Taylor A, Jones GT (2017) EULAR revised recommendations for the management of fibromyalgia. Ann Rheum Dis 76:318-328

97. Maier C, Kindler D (2016) Analgetika. In: Maier C, Bingel U, Diener HC (Hrsg) Lehrbuch der Schmerzmedizin, 5. Aufl. Elsevier, München, S393-411

98. Maier C (2018) Wieder ein "War on Drugs". Ein Krieg gegen Süchtige und ein "roll-back" für opioidbedürftige Patienten? Schmerz 32:167-170

99. Manchikanti L, Helm S 2nd, Fellows B, Janata JW, Pampati V, Grider JS, Boswell MV (2012) Opioid epidemic in the United States. Pain Physician 15:ES9-38

100. MarschallU,L'hoestH, Radbruch L, HäuserW(2016) Long-term opioid therapy for chronic non-cancer pain in Germany. Eur J Pain 20:767-776 (Erratum in: Eur J Pain 2017;21:1774)

101. Mayerle J, Hoffmeister A, Werner J, Witt $H$, Lerch MM, Mössner J (2013) Chronic pancreatitis-Definition, etiology, investigation and treatment. Dtsch Arztebl Int 110:387-393

102. Megale RZ, Deveza LA, Blyth FM, Naganathan V, Ferreira PH, McLachlan AJ, Ferreira ML (2018) Efficacy and safety of oral and transdermal opioid analgesics for musculoskeletal pain in older adults: a systematic review of randomized, placebocontrolled trials. JPain 19:475.e1-475.e24

103. Moisset X, Trouvin AP, Tran VT, Authier N, VergneSalle P, Piano V, Martinez V (2016) Use of strong opioids in chronic non-cancer pain in adults. Evidence-based recommendations from the French Society for the Study and Treatment of Pain. Presse Med 45(4 Pt 1):447-462

104. Morlion BJ, Mueller-Lissner SA, Vellucci R, Leppert W, Coffin BC, Dickerson SL, O'Brien T (2018) Oral prolonged-release oxycodone/Naloxone for managing pain and opioid-induced constipation: a review of the evidence. Pain Pract 18:647-665

105. Müller-Lissner S (2013) Opiate induced constipation-mechanisms, relevance and treatment. Dtsch Med Wochenschr 138(43):2207-2211

106. Nelson AE, Allen KD, Golightly YM, Goode AP, Jordan JM (2014) A systematic review of recommendations and guidelines for the management of osteoarthritis: The chronic osteoarthritis management initiative of the U.S. Bone and Joint Initiative. Semin Arthritis Rheum 43:701-712

107. Neuman MD, Bateman BT, Wunsch H (2019) Inappropriate opioid prescription after surgery. Lancet 393:1547-1557

108. Nielsen S, Larance B, Degenhardt L, Gowing L, Kehler C, Lintzeris N (2016) Opioid agonist treatment for pharmaceutical opioid dependent people. Cochrane Database Syst Rev 5:CD11117

109. Niemann T, Madsen LG, Larsen S, Thorsgaard N (2000) Opioid treatment of painful chronic pancreatitis. Int J Pancreatol 27:235-240 
110. O'Brien EM, Staud RM, Hassinger AD, McCulloch RC Craggs JG, Atchison JW, Price DD, Robinson ME (2010) Patient-centered perspective on treatment outcomes in chronic pain. Pain Med 11:6-15

111. OECD (2019) Addressing problematic opioid use in OECD countries. https://www.oecd.org/ health/addressing-problematic-opioid-use-inoecd-countries-a18286f0-en.htm. Zugegriffen: 2 . Aug. 2019

112. Okie S (2010) A flood of opioids, a rising tide of deaths. N Engl J Med 363:1981-1985 (Erratum in: N Engl J Med 2011;364:290)

113. Olesen SS, Poulsen JL, Broberg MC, Madzak A, Drewes AM (2016) Opioid treatment and hypoalbuminemia are associated with increased hospitalisation rates in chronic pancreatitis outpatients. Pancreatology 16:807-813

114. Oliveira CB, Maher CG, Pinto RZ, Traeger AC, Lin CC, Chenot JF, van Tulder M, Koes BW (2018) Clinical practice guidelines for the management of nonspecific low back pain in primary care: an updated overview. Eur Spine J 27:2791-2803

115. Park TW, Saitz R, Ganoczy D, Ilgen MA, Bohnert AS (2015) Benzodiazepine prescribing patterns and deaths from drug overdose among US veterans receiving opioid analgesics: case-cohort study. BMJ 350:h2698

116. Petri H, Grandt D (2016) Interaktionen der Opioidanalgetika auf Ebene der Biotransformation. Schmerz 30:519-525

117. Pergolizzi J, Aloisi AM, Dahan A, Filitz J, Langford R, Likar R, Mercadante S, Morlion B, Raffa RB, Sabatowski $R$, Sacerdote $P$, Torres LM, Weinbroum AA (2010) Current knowledge of buprenorphine and its unique pharmacological profile. Pain Pract 10:428-450

118. Petzke F, Welsch P, Klose P, Sommer C, Häuser W (2020) Opioids for chronic low back pain. An updated systematic review and meta-analysis of efficacy, tolerability and safety in randomized placebo-controlled studies of at least four weeks double-blind duration. Eur J Pain 24(3):497-517

119. Poulsen, Arendt-Nielsen, Brosen K et al (1996) The hypoalgesic effect of tramadol in relation to CyP2D6. Clin Pharmacol Ther 60:636-644

120. PreißJC, Bokemeyer B, Buhr HJ, DignaßA, HäuserW, Hartmann F, Herrlinger KR, Kaltz B, Kienle P, Kruis W, Kucharzik T, Langhorst J, Schreiber S, Siegmund B, Stallmach A, Stange EF, Stein J, Hoffmann JC, German Society of Gastroenterology (2014) Updated German clinical practice guideline on "Diagnosis and treatment of Crohn's disease" 2014. ZGastroenterol 52:1431-1484

121. Purdue Pharma (2005) MS Contin [package insert]. Conn, Stamford

122. Ranapurwala SI, Naumann RB, Austin AE, Dasgupta N, Marshall SW (2019) Methodologic limitations of prescription opioid safety research and recommendations for improving the evidence base. Pharmacoepidemiol Drug Saf 28:4-12

123. Ratiopharm (2018) Fachinformation Morphin retard Tabletten. https://www.ratiopharm.de/ assets/products/de/label/Morphin-ratiopharm \%20Retardtabletten\%20-\%202.pdf?pzn=8911824. Zugegriffen: 9.Jan. 2020

124. Ratz D, Wiitala W, Badr MS, Burns J, Chowdhuri S (2018) Correlates and consequences of central sleep apnea in a national sample of US veterans. Sleep. https://doi.org/10.1093/sleep/zsy058

125. Rauck RL (2009) What is the case for prescribing long-acting opioids over shortacting opioids for patients with chronic pain? A critical review. Pain Pract 9:468-479
126. Ray WA, Chung CP, Murray KT, Hall K, Stein CM (2017) Prescription of long-acting opioids and mortality in patients with chronic noncancer pain JAMA 315:2415-2423

127. Reda H, Greene K, Rice FL et al (2013) Natural history of herpes zoster: late follow-up of 3.9 years $(n=43)$ and 7.7 years $(n=10)$. Pain 154:2227-2233

128. Roenneberg C, Sattel H, Schaefert R, Henningsen $P$, Hausteiner-Wiehle C (2019) Funktionelle Körperbeschwerden. Dtsch Arztebl Int 116:553-560

129. Rosner B, Neicun J, Yang JC, Roman-Urrestarazu A (2019) Opioid prescription patterns in Germany and the global opioid epidemic: systematic review of available evidence. PLoSONE 14:e221153

130. Russell IJ, Kamin M, Bennett RM, Schnitzer TJ, Green JA, Katz WA (2000) Efficacy of tramadol in treatment of pain in fibromyalgia.J Clin Rheumatol 6:250-257

131. Santos J, Alarcão J, Fareleira F, Vaz-Carneiro A, Costa J (2015) Chronic musculoskeletal pain in adults. Cochrane Database Syst Rev 5:CD9923

132. Savage S, Covington E, Heit H, Hunt J, Joranson D, Schnoll S (2001) Definitions related to the use of opioids for the treatment of pain: a consensus document from the American Academy of Pain Medicine, the American Pain Society, and the American Society of Addiction Medicine. American Pain Society Advocacy and Policy

133. Schenk M, Wirz S (2015) Serotoninsyndrom und medikamentöse Schmerztherapie. Schmerz 2:229-251

134. Scholl L, Seth P, Kariisa M, Wilson N, Baldwin G (2018) Drug and opioid-involved overdose deaths-United States, 2013-2017. Mmwr Morb Mortal Wkly Rep 67:1419-1427

135. Scholten W, Simon O, Maremmani I, Wells C, Kelly JF, Hämmig R, Radbruch L (2017) Access to treatment with controlled medicines rationale and recommendations for neutral, precise, and respectful language. Public Health 153:147-153

136. Schurig AM, Böhme M, Just KS, Scholl C, Dormann H, Plank-Kiegele B, Seufferlein T, Gräff I, Schwab M, Stingl JC (2018) Adverse drug reactions (ADR) and emergencies. Dtsch Arztebl Int 115:251-258

137. Schwarzer A, Aichinger-Hinterhofer M, Maier C, Vollert J, Walther JW (2015) Sleep-disordered breathing decreases after opioid withdrawal: results of a prospective controlled trial. Pain 156:2167-2174

138. Schwarzer A, Scherbaum N, Kindler D, Maier C (2017) Diagnostik, Therapie und Prävention der Opioidabhängigkeit. In: Maier C, Diener HC, Bingel U (Hrsg) Schmerzmedizin. Urban \& Fischer München, S338-352

139. Seitz NN, Lochbühler K, Atzendorf J, Rauschert C, Pfeiffer-Gerschel T, Kraus L (2019) Trends in substance use nand related disorders - analysis of the Epidemiological Survey of Substance Abuse. 1995 to 2018. Dtsch Arztebl Int 116:585-519

140. Seppala LJ, van de Glind EMM, Daams JG, Ploegmakers KJ, de Vries M, Wermelink AMAT, van der Velde N, EUGMS Task and Finish Group on FallRisk-Increasing Drugs (2018) Fall-risk-increasing drugs: a systematic review and meta-analysis: III. Others. J Am Med Dir Assoc 19(372):372.e1-372.e8

141. Singh VK, Yadav D, Garg PK (2019) Diagnosis and management of chronic pancreatitis: a review. JAMA 322:2422-2434

142. Smith HS, Laufer A (2014) Opioid induced nausea and vomiting. Eur JPharmacol 722:67-78

143. Smith SR, Deshpande BR, Collins JE, Katz JN, Losina $E$ (2016) Comparative pain reduction of oral non-steroidal anti-inflammatory drugs and opioids for knee osteoarthritis: systematic analytic review. Osteoarthritis Cartilage 24:962-972

144. Sommer C, Alten R, Bär KJ, Bernateck M, Brückle W, Friedel E, Henningsen P, Petzke F, Tölle T, Üçeyler N, Winkelmann A, Häuser W (2017) Drug therapy of fibromyalgia syndrome: updated guidelines 2017 and overview of systematic review articles. Schmerz 31:274-284

145. Sommer C, Klose P, Welsch P, Petzke F, Häuser W (2020) Opioids for chronic non-cancer neuropathic pain. An updated systematic review and metaanalysis of efficacy, tolerability and safety in randomized placebo-controlled studies of at least four weeks duration. Eur J Pain 24(1):3-18

146. Sun EC, Dixit A, Humphreys K, Darnall BD, Baker LC, Mackey S (2017) Association between concurrent use of prescription opioids and benzodiazepines and overdose: retrospective analysis. BMJ 356:j760

147. Trenkwalder C, Beneš H, Grote L, García-Borreguero D, Högl B, Hopp M, Bosse B, Oksche A, Reimer K Winkelmann J, Allen RP, Kohnen R, RELOXYN Study Group (2013) Prolonged release oxycodonenaloxone for treatment of severe restless legs syndrome after failure of previous treatment: a double-blind, randomised, placebo-controlled trial with an open-label extension. Lancet Neurol 12:1141-1150

148. Trenkwalder $C$, Chaudhuri KR, Martinez-Martin $P$, Rascol O, Ehret R, Vališ M, Sátori M, KrygowskaWajs A, Marti MJ, Reimer K, Oksche A, Lomax M DeCesare J, Hopp M (2015) Prolonged-release oxycodone-naloxone for treatment of aevere pain in patients with Parkinson's disease (PANDA): a double-blind, randomised, placebo-controlled trial. Lancet Neurol 14:1161-1170

149. Von Korff M, Saunders K, Thomas Ray G, Boudreau D, Campbell C, Merrill J, Sullivan MD, Rutter CM, Silverberg MJ, Banta-Green C, Weisner C (2008) De facto long-term opioid therapy for noncancer pain. Clin J Pain 24:521-527

150. Wang H, Akbar M, Weinsheimer N, Gantz S, Schiltenwolf M (2011) Longitudinal observation of changes in pain sensitivity during opioid tapering in patients with chronic low-back pain. Pain Med 12:1720-1726

151. Wang AMQ, Retrouvey H, Wanzel KR (2018) Addressing the opioid epidemic: a review of the role of plastic surgery. Plast Reconstr Surg 14:1295-1301

152. Wasner G, Deuschl G (2012) Pains in Parkinson disease-many syndromes under one umbrella. Nat Rev Neurol 8:284-294

153. Wedmann F, Himmel W, Nau R (2019) Medication and medical diagnosis as risk factors for falls in older hospitalized patients. Eur J Clin Pharmacol 75(8):1117-1124

154. Welsch $P$, Üçeyler N, Klose P, Walitt B, Häuser W (2018) Serotonin and noradrenaline reuptake inhibitors (SNRIs) for fibromyalgia. Cochrane Database Syst Rev 2:CD10292

155. Welsch P, Klose P, Petzke F, Häuser W (2020) Opioids for chronic osteoarthritis pain. An updated systematic review and metaanalysis of efficacy, tolerability and safety in randomized placebocontrolled studies of at least four weeks doubleblind duration. Eur JPain 24(4):685-703

156. Wiese AD, Griffin MR, Stein CM, Mitchel EF Jr, Grijalva CG (2016) Opioid analgesics and the risk of serious infections among patients with rheumatoid arthritis: a self-controlled case series study. Arthritis Rheumatol 68:323-331

157. Wiese AD, Griffin MR, Schaffner W, Stein CM, Greevy RA, Mitchel EF, Grijalva CG (2019) Longacting Opioid use and the risk of serious infections: 
a retrospective cohort study. Clin Infect Dis 68:1862-1869

158. Zella MAS, May C, Müller T, Ahrens M, Tönges L, Gold R, Marcus K, Woitalla D (2019) Landscape of pain in Parkinson's disease: impact of gender differences. Neurol Res 41:87-97

159. Zeng C, Dubreuil M, LaRochelle MR, Lu N, Wei J, Choi HK, Lei G, Zhang Y (2019) Association of tramadol with all-cause mortality among patients with osteoarthritis. JAMA 321:969-982 